\title{
23. UNDERWAY GEOPHYSICAL DATA: NAVIGATION, BATHYMETRY, MAGNETICS, AND SEISMIC PROFILES
}

\author{
S.O. Schlanger, University of California, Riverside, California \\ and \\ E.L. Winterer, Scripps Institution of Oceanography, La Jolla, California
}

\section{INTRODUCTION AND METHODS}

The underway data collected on Leg 33 includes magnetic, bathymetric, and seismic profiles and navigational records. Much of the material presented in this chapter was compiled from shipboard records and processed by S. Smith, U. Albright, B. Long, L. Bunch, and G. Psaropolus of The Underway Data Processing Group, Scripps Institution of Oceanography Geological Data Center (for information on the availability and reproduction costs of these data, contact the Scientific Information Facility, Deep Sea Drilling Project, S10, La Jolla, Calif. 92037). The recording of the basic data aboard Glomar Challenger was done under the supervision of the Laboratory Officer for Leg 33, Robert Iuliucci.

Methods used are outlined below:

Navigation: Satellite fixes and course and speed changes were encoded aboard Glomar Challenger from data given in the underway geophysical log. The data were keypunched on shore, and put through a navigation smoothing program, edited on the basis of reasonable ship and drift velocities, and a deck of corrected navigation points punched out for later merging in the depth and magnetic data. Table 1 includes time, position, satellite fix and drift velocity data, and course and speed changes. The ship's track and time ticks to which the seismic profiles (see Figure 2a-dd) are keyed are shown in Figure 1 (in pocket at back of volume), a bathymetric chart of the region traversed during Leg 33 .

Magnetics: The magnetics (in gammas), scaled from analog records produced by a Varian proton-precession magnetometer towed 200-300 meters behind the ship, were recorded at sea in the underway geophysical logbook at 5-min intervals. These data were keypunched on shore, put through a profile program, and edited by comparison to the original analog records. Available from the Scientific Information Facility are plots of magnetic anomaly profiles along the ship's track shown in Figure 1, from values retrieved at approximately 1 mile spacing; the regional field was removed using the 1965 International Geomagnetic Reference Field. In this chapter, the magnetic data are shown as profiles keyed to the navigation, depth, and seismic profile records in Figure 3a-g.

Depth: The depths scaled from the fathograms made on a Gifft 12-kHz PDR calibrated to an 800 fathom/sec sound velocity $(1.463 \mathrm{~km} / \mathrm{sec})$ were recorded at sea in the underway geophysical logbook at 5-min intervals. The depths were keypunched on shore and edited in the same fashion as the magnetics data. The bathymetric profiles so generated are shown in Figure 3a-g below the magnetic profiles. Available from the Scientific Information Facility are depth plots, in fathoms (using an assumed 800 fathom/sec sound velocity) at approximately 1 mile spacing, plotted at $4 "$ /degree with standard U.S. Navy Oceanographic Office BC series boundaries.

Seismic profiles: The energy sources used were 2 Bolt $600 \mathrm{~A}$ airguns, of 30 and $40 \mathrm{in}^{3}$. Returns were recorded on 2 EDO Western Model 333 recorders. The number 1 EDO was kept on 10-sec sweep, with the band pass filter generally set for a $160-320 \mathrm{~Hz}$ range; occasionally, the $80-160 \mathrm{~Hz}$ or $40-160 \mathrm{~Hz}$ band was recorded. On these profiles (see Figure 2a-dd), which are photographs of the original records, are shown the vertical scale in seconds of two-way travel time, local time, and the ship's course and speed; drill sites are also indicated. Each profile photograph has a roll and number that is keyed to the ship's track on Figure 1 and the magnetic and bathymetric profiles shown on Figure 3a-g. The number 2 EDO was set for a 4-sec sweep with a 2-6 sec trigger delay deepending on the water depth; a 160-320 $\mathrm{Hz}$ band pass setting was generally used. These 4-sec sweep records, annotated in terms of drill-site stratigraphy, acoustistratigraphy, physiography, and structure are shown as Figure $4 \mathrm{a}-\mathrm{f}$.

Sonobuoy runs were made at the various sites. See the individual Site Reports for methods and results.

\section{RESULTS AND DISCUSSION}

\section{Honolulu to Site 314 (Figure 4a)}

After leaving Honolulu Glomar Challenger steamed west-southwest over the probable distal southern edge of a turbidite fan of Kaula-Kauai Island provenance (0000 4 November 1973). These sediments appear to be ponded against the slightly higher standing, topographically rougher northwest end of the Hawaiian Arch. Records obtained on Leg 17 (Raff, 1973; profiles 32,33 , and 34) show how the turbidites have smoothed over the rougher basement topography. The northeastern end of the Hawaiian Arch, which seems to be interrupted by an extension of the Molokai Fracture Zone (Figure 1), shows rough topography (0000 4 November 1973 to $\sim 18004$ November 1973). Locally $0.1-0.5 \mathrm{sec}$ of an upper transparent layer lies over possible older sedimentary sections represented by a vague reflector $\sim 0.1-0.2 \mathrm{sec}$ thick ( 1100 4 November 1973). Bottom water, flowing east towards the Hawaiian Islands through passes in the Line Islands south of Horizon Guyot (Normark and Spiess, in press), may be eroding the bottom sediments. At 00005 November 1973 we passed $\sim 105 \mathrm{n} \mathrm{mi} \mathrm{north-northwest} \mathrm{of} \mathrm{Site} 68$ 
TABLE 1

Navigation Data, Leg 33

\begin{tabular}{|c|c|c|c|c|c|c|c|c|c|c|c|c|c|}
\hline & & & & & & & & & Dri & & & & \\
\hline DA & MO & YR & Time & Latitude & Longitude & Dist. & Speed & Course & Speed & Hed. & Speed & Course & Comment \\
\hline 3 & 11 & 1973 & 0430 & $21^{\circ} 16.2^{\prime}$ & $-157^{\circ} 51.0^{\prime}$ & 0.0 & 8.1 & 244 & 0.4 & 71 & 8.5 & 244 & DR \\
\hline 3 & 11 & 1973 & 500 & $21^{\circ} 14.4^{\prime}$ & $-157^{\circ} 54.9^{\prime}$ & 4.1 & 8.6 & 247 & 0.4 & 321 & 8.5 & 244 & DR \\
\hline 3 & 11 & 1973 & 544 & $21^{\circ} 11.9^{\prime}$ & $-158^{\circ} \quad 1.1^{\prime}$ & 10.4 & 9.0 & 244 & 0.5 & 248 & 8.5 & 244 & SATL \\
\hline 3 & 11 & 1973 & 716 & $21^{\circ} 5.9^{\prime}$ & $-158^{\circ} 14.4^{\prime}$ & 24.1 & 9.2 & 244 & 0.7 & 250 & 8.5 & 244 & SATL \\
\hline 3 & 11 & 1973 & 830 & $21^{\circ} 1.0^{\prime}$ & $-158^{\circ} 25.4^{\prime}$ & 35.5 & 9.7 & 246 & 1.3 & 257 & 8.5 & 244 & SATL \\
\hline 3 & 11 & 1973 & 96 & $20^{\circ} 58.6^{\prime}$ & $-158^{\circ} 31.1^{\prime}$ & 41.3 & 9.5 & 242 & 1.1 & 226 & 8.5 & 244 & SATL \\
\hline 3 & 11 & 1973 & 1016 & $20^{\circ} 53.4^{\prime}$ & $-158^{\circ} 41.6^{\prime}$ & 52.4 & 9.3 & 241 & 0.9 & 212 & 8.5 & 244 & SATL \\
\hline 3 & 11 & 1973 & 1152 & $20^{\circ} 46.2^{\prime}$ & $-158^{\circ} 55.5^{\prime}$ & 67.3 & 8.9 & 244 & 0.4 & 235 & 8.5 & 244 & SATL \\
\hline 3 & 11 & 1973 & 123 & $20^{\circ} 45.5^{\prime}$ & $-158^{\circ} 57.1^{\prime}$ & 68.9 & 10.4 & 244 & 0.4 & 235 & 10.0 & 244 & $\mathrm{C} / \mathrm{S}$ \\
\hline 3 & 11 & 1973 & 130 & $20^{\circ} 41.1^{\prime}$ & $-159^{\circ} \quad 6.5^{\prime}$ & 78.8 & 10.4 & 247 & 0.4 & 235 & 10.0 & 247 & $\mathrm{C} / \mathrm{C}$ \\
\hline 3 & 11 & 1973 & 160 & $20^{\circ} 28.7^{\prime}$ & $-159^{\circ} 37.0^{\prime}$ & 109.9 & 10.4 & 243 & 0.4 & 235 & 10.0 & 243 & $\mathrm{C} / \mathrm{C}$ \\
\hline 3 & 11 & 1973 & 172 & $20^{\circ} 23.8^{\prime}$ & $-159^{\circ} 47.2^{\prime}$ & 120.7 & 10.2 & 243 & 0.2 & 233 & 10.0 & 243 & SATL \\
\hline 3 & 11 & 1973 & 1846 & $20^{\circ} 15.7^{\prime}$ & $-160^{\circ} \quad 4.0^{\prime}$ & 138.4 & 9.7 & 243 & 0.3 & 50 & 10.0 & 243 & SATL \\
\hline 3 & 11 & 1973 & 1952 & $20^{\circ} 10.9^{\prime}$ & $-160^{\circ} 14.2^{\prime}$ & 149.1 & 10.4 & 250 & 1.3 & 318 & 10.0 & 243 & SATL \\
\hline 3 & 11 & 1973 & 2022 & $20^{\circ} 9.1^{\prime}$ & $-160^{\circ} 19.4^{\prime}$ & 154.3 & 10.0 & 245 & 0.3 & 334 & 10.0 & 243 & SATL \\
\hline 3 & 11 & 1973 & 2138 & $20^{\circ} \quad 3.7^{\prime}$ & $-160^{\circ} 31.6^{\prime}$ & 166.9 & 10.2 & 244 & 0.3 & 288 & 10.0 & 243 & SATL \\
\hline 3 & 11 & 1973 & 220 & $20^{\circ} \quad 2.1^{\prime}$ & $-160^{\circ} 35.2^{\prime}$ & 170.7 & 9.7 & 244 & 0.3 & 288 & 9.5 & 243 & $\mathrm{C} / \mathrm{S}$ \\
\hline 3 & 11 & 1973 & 2330 & $19^{\circ} 55.8^{\prime}$ & $-160^{\circ} 49.2^{\prime}$ & 185.3 & 9.7 & 242 & 0.3 & 288 & 9.5 & 241 & $\mathrm{C} / \mathrm{C}$ \\
\hline 4 & 11 & 1973 & 00 & $19^{\circ} 53.5^{\prime}$ & $-160^{\circ} 53.8^{\prime}$ & 190.2 & 9.7 & 242 & 0.3 & 288 & 9.5 & 241 & \\
\hline 4 & 11 & 1973 & 128 & $19^{\circ} 46.9^{\prime}$ & $-161^{\circ} 7.2^{\prime}$ & 204.4 & 9.6 & 244 & 0.6 & 320 & 9.5 & 241 & SATL \\
\hline 4 & 11 & 1973 & 20 & $19^{\circ} 44.7^{\prime}$ & $-161^{\circ} 12.1^{\prime}$ & 209.5 & 10.1 & 244 & 0.6 & 320 & 10.0 & 241 & $\mathrm{C} / \mathrm{S}$ \\
\hline 4 & 11 & 1973 & 216 & $19^{\circ} 43.5^{\prime}$ & $-161^{\circ} 14.7^{\prime}$ & 212.2 & 9.3 & 239 & 0.8 & 88 & 10.0 & 241 & SATL \\
\hline 4 & 11 & 1973 & 358 & $19^{\circ} 35.3^{\prime}$ & $-161^{\circ} 29.1^{\prime}$ & 288.1 & 9.8 & 238 & 0.5 & 133 & 10.0 & 241 & SATL \\
\hline 4 & 11 & 1973 & 431 & $19^{\circ} 32.4^{\prime}$ & $-161^{\circ} 34.0^{\prime}$ & 233.5 & 9.8 & 241 & 0.5 & 133 & 10.0 & 244 & $\mathrm{C} / \mathrm{C}$ \\
\hline 4 & 11 & 1973 & 86 & $19^{\circ} 15.4^{\prime}$ & $-162^{\circ} \quad 6.6^{\prime}$ & 268.7 & 10.1 & 244 & 0.1 & 202 & 10.0 & 244 & SATL \\
\hline 4 & 11 & 1973 & 90 & $19^{\circ} 11.4^{\prime}$ & $-162^{\circ} 15.2^{\prime}$ & 277.7 & 10.1 & 247 & 0.1 & 202 & 10.0 & 247 & $\mathrm{C} / \mathrm{C}$ \\
\hline 4 & 11 & 1973 & 924 & $19^{\circ} \quad 9.8^{\prime}$ & $-162^{\circ} 19.1^{\prime}$ & 281.7 & 9.9 & 246 & 0.3 & 127 & 10.0 & 247 & SATL \\
\hline 4 & 11 & 1973 & 116 & $19^{\circ} 2.9^{\prime}$ & $-162^{\circ} 35.3^{\prime}$ & 298.5 & 10.0 & 248 & 0.1 & 359 & 10.0 & 247 & SATL \\
\hline 4 & 11 & 1973 & 1252 & $18^{\circ} 56.2^{\prime}$ & $-162^{\circ} 52.5^{\prime}$ & 316.1 & 9.7 & 245 & 0.5 & 125 & 10.0 & 247 & SATL \\
\hline 4 & 11 & 1973 & 180 & $18^{\circ} 34.7^{\prime}$ & $-163^{\circ} 40.2^{\prime}$ & 366.1 & 9.4 & 243 & 0.9 & 118 & 10.0 & 247 & SATL \\
\hline 4 & 11 & 1973 & 1915 & $18^{\circ} 29.3^{\prime}$ & $-163^{\circ} 51.3^{\prime}$ & 377.9 & 9.4 & 245 & 0.9 & 118 & 10.0 & 249 & $\mathrm{C} / \mathrm{C}$ \\
\hline 4 & 11 & 1973 & 1928 & $18^{\circ} 28.4^{\prime}$ & $-163^{\circ} 53.2^{\prime}$ & 380.0 & 9.9 & 245 & 0.8 & 146 & 10.0 & 249 & SATL \\
\hline 4 & 11 & 1973 & 2046 & $18^{\circ} 22.9^{\prime}$ & $-164^{\circ} \quad 5.4^{\prime}$ & 392.8 & 9.3 & 245 & 1.0 & 113 & 10.0 & 249 & SATL \\
\hline 4 & 11 & 1973 & 2232 & $18^{\circ} 15.9^{\prime}$ & $-164^{\circ} 21.1^{\prime}$ & 409.3 & 9.3 & 247 & 0.8 & 91 & 10.0 & 249 & SATL \\
\hline 4 & 11 & 1973 & 2252 & $18^{\circ} 14.7^{\prime}$ & $-164^{\circ} 24.1^{\prime}$ & 412.4 & 9.5 & 247 & 0.6 & 106 & 10.0 & 249 & SATL \\
\hline 5 & 11 & 1973 & 00 & $18^{\circ} 10.4^{\prime}$ & $-164^{\circ} 34.6^{\prime}$ & 423.2 & 9.5 & 247 & 0.6 & 106 & 10.0 & 249 & \\
\hline 5 & 11 & 1973 & 036 & $18^{\circ} 8.2^{\prime}$ & $-164^{\circ} 40.1^{\prime}$ & 428.9 & 9.4 & 249 & 0.6 & 71 & 10.0 & 249 & SATL \\
\hline 5 & 11 & 1973 & 258 & $\begin{array}{lll}18^{\circ} & 0.2^{\prime}\end{array}$ & $\begin{array}{lll}-165^{\circ} & 1.9^{\prime}\end{array}$ & 451.1 & 9.9 & 251 & 0.4 & 357 & 10.0 & 249 & SATL \\
\hline 5 & 11 & 1973 & 40 & $17^{\circ} 56.9^{\prime}$ & $-165^{\circ} 12.1^{\prime}$ & 461.3 & 9.9 & 247 & 0.4 & 357 & 10.0 & 245 & $\mathrm{C} / \mathrm{C}$ \\
\hline 5 & 11 & 1973 & 544 & $17^{\circ} 50.2^{\prime}$ & $-165^{\circ} 28.6^{\prime}$ & 478.4 & 9.2 & 246 & 0.8 & 59 & 10.0 & 245 & SATL \\
\hline 5 & 11 & 1973 & 640 & $17^{\circ} 46.6^{\prime}$ & $-165^{\circ} 36.8^{\prime}$ & 487.0 & 9.2 & 244 & 0.8 & 59 & 10.0 & 244 & $\mathrm{C} / \mathrm{C}$ \\
\hline 5 & 11 & 1973 & 712 & $17^{\circ} 44.5^{\prime}$ & $-165^{\circ} 41.5^{\prime}$ & 492.0 & 10.2 & 244 & 0.2 & 262 & 10.0 & 244 & SATL \\
\hline 5 & 11 & 1973 & 1018 & $17^{\circ} 30.8^{\prime}$ & $-166^{\circ} 11.5^{\prime}$ & 523.7 & 10.0 & 243 & 0.3 & 164 & 10.0 & 244 & SATL \\
\hline 5 & 11 & 1973 & 1045 & $17^{\circ} 28.7^{\prime}$ & $-166^{\circ} 15.7^{\prime}$ & 528.2 & 10.1 & 242 & 0.3 & 164 & 10.0 & 243 & $\mathrm{C} / \mathrm{C}$ \\
\hline 5 & 11 & 1973 & 124 & $17^{\circ} 22.4^{\prime}$ & $-166^{\circ} 27.9^{\prime}$ & 541.4 & 10.5 & 242 & 0.5 & 216 & 10.0 & 243 & SATL \\
\hline 5 & 11 & 1973 & 1430 & $17^{\circ} 10.3^{\prime}$ & $-166^{\circ} 51.4^{\prime}$ & 566.9 & 10.1 & 242 & 0.2 & 184 & 10.0 & 243 & SATL \\
\hline 5 & 11 & 1973 & 1714 & $16^{\circ} 57.4^{\prime}$ & $-167^{\circ} 16.9^{\prime}$ & 594.5 & 10.1 & 243 & 0.2 & 213 & 10.0 & 243 & SATL \\
\hline 5 & 11 & 1973 & 1740 & $16^{\circ} 55.4^{\prime}$ & $-167^{\circ} 21.0^{\prime}$ & 598.9 & 10.1 & 245 & 0.2 & 213 & 10.0 & 246 & $\mathrm{C} / \mathrm{C}$ \\
\hline 5 & 11 & 1973 & 1838 & $16^{\circ} 51.3^{\prime}$ & $-167^{\circ} 30.3^{\prime}$ & 608.7 & 10.3 & 246 & 0.3 & 259 & 10.0 & 246 & SATL \\
\hline $\begin{array}{l}5 \\
5\end{array}$ & 11 & 1973 & 1912 & $16^{\circ} 49.0^{\prime}$ & $-167^{\circ} 35.9^{\prime}$ & 614.5 & 10.3 & 245 & 0.3 & 259 & 10.0 & 244 & $\mathrm{C} / \mathrm{C}$ \\
\hline 5 & 11 & 1973 & 200 & $16^{\circ} 45.4^{\prime}$ & $-167^{\circ} 43.7^{\prime}$ & 622.8 & 10.9 & 243 & 0.9 & 229 & 10.0 & 244 & SATL \\
\hline 5 & 11 & 1973 & 2142 & $16^{\circ} 36.9^{\prime}$ & $\begin{array}{lll}-168^{\circ} & 0.9^{\prime}\end{array}$ & 641.4 & 9.8 & 240 & 0.6 & 138 & 10.0 & 244 & SATL \\
\hline 5 & 11 & 1973 & 2250 & $16^{\circ} 31.4^{\prime}$ & $-168^{\circ} 11.0^{\prime}$ & 652.5 & 9.8 & 242 & 0.6 & 138 & 10.0 & 246 & $\mathrm{C} / \mathrm{C}$ \\
\hline 5 & 11 & 1973 & 2348 & $16^{\circ} 27.0^{\prime}$ & $-168^{\circ} 19.8^{\prime}$ & 662.0 & 10.3 & 246 & 0.3 & 249 & 10.0 & 246 & SATL \\
\hline 6 & 11 & 1973 & 00 & $16^{\circ} 26.2^{\prime}$ & $-168^{\circ} 21.8^{\prime}$ & 664.1 & 10.3 & 246 & 0.3 & 249 & 10.0 & 246 & \\
\hline 6 & 11 & 1973 & 025 & $16^{\circ} 24.4^{\prime}$ & $-168^{\circ} 25.8^{\prime}$ & 668.4 & 10.3 & 252 & 0.3 & 249 & 10.0 & 252 & $\mathrm{C} / \mathrm{C}$ \\
\hline 6 & 11 & 1973 & 115 & $16^{\circ} 21.8^{\prime}$ & $-168^{\circ} 34.4^{\prime}$ & 677.0 & 10.3 & 246 & 0.3 & 249 & 10.0 & 246 & $\mathrm{C} / \mathrm{C}$ \\
\hline 6 & 11 & 1973 & 136 & $16^{\circ} 20.3^{\prime}$ & $-168^{\circ} 37.8^{\prime}$ & 680.6 & 10.3 & 249 & 0.6 & 310 & 10.0 & 246 & SATL \\
\hline 6 & 11 & 1973 & 24 & $16^{\circ} 18.6^{\prime}$ & $-168^{\circ} 42.4^{\prime}$ & 685.3 & 9.5 & 149 & 0.6 & 310 & 10.0 & 248 & $\mathrm{C} / \mathrm{C}$ \\
\hline 6 & 11 & 1973 & 29 & $16^{\circ} 17.9^{\prime}$ & $-168^{\circ} 42.0^{\prime}$ & 686.1 & 8.3 & 149 & 0.6 & 310 & 8.8 & 148 & $\mathrm{C} / \mathrm{S}$. \\
\hline 6 & 11 & 1973 & 52 & $15^{\circ} 57.4^{\prime}$ & $-168^{\circ} 29.3^{\prime}$ & 710.0 & 8.9 & 147 & 0.2 & 84 & 8.8 & 148 & SATL \\
\hline 6 & 11 & 1973 & 526 & $15^{\circ} 54.4^{\prime}$ & $-168^{\circ} 27.3^{\prime}$ & 713.5 & 6.5 & 146 & 0.2 & 84 & 6.4 & 148 & $\mathrm{C} / \mathrm{S}$ \\
\hline 6 & 11 & 1973 & 549 & $15^{\circ} 52.4^{\prime}$ & $-168^{\circ} 25.8^{\prime}$ & 716.0 & 9.0 & 318 & 0.2 & 84 & 9.1 & 317 & $\mathrm{C} / \mathrm{CS}$ \\
\hline 6 & 11 & 1973 & 555 & $15^{\circ} 53.0^{\prime}$ & $-168^{\circ} 26.5^{\prime}$ & 716.9 & 6.9 & 318 & 0.2 & 84 & 7.0 & 317 & $\mathrm{C} / \mathrm{S}$ \\
\hline 6 & 11 & 1973 & 558 & $15^{\circ} 53.3^{\prime}$ & $-168^{\circ} 26.7^{\prime}$ & 717.3 & 6.0 & 319 & 0.2 & 84 & 6.1 & 317 & $\mathrm{C} / \mathrm{S}$ \\
\hline 6 & 11 & 1973 & 60 & $15^{\circ} 53.4^{\prime}$ & $-168^{\circ} 26.8^{\prime}$ & 717.5 & 6.9 & 318 & 0.2 & 84 & 7.0 & 317 & $\mathrm{C} / \mathrm{S}$ \\
\hline 6 & 11 & 1973 & 616 & $15^{\circ} 54.8^{\prime}$ & $-168^{\circ} 28.1^{\prime}$ & 719.3 & 7.0 & 317 & 0.0 & 0 & 7.0 & 317 & S314 \\
\hline 6 & 11 & 1973 & 616 & $15^{\circ} 54.8^{\prime}$ & $-168^{\circ} 28.1^{\prime}$ & 719.3 & 0.0 & 0 & 0.0 & 0 & 0.0 & 500 & STOP \\
\hline 8 & 11 & 1973 & 142 & $15^{\circ} 54.8^{\prime}$ & $-168^{\circ} 28.1^{\prime}$ & 719.3 & 1.1 & 293 & 1.1 & 293 & 0.0 & 500 & DEP \\
\hline 8 & 11 & 1973 & 142 & $15^{\circ} 54.8^{\prime}$ & $-168^{\circ} 28.1^{\prime}$ & 719.3 & 7.9 & 144 & 1.1 & 293 & 8.8 & 140 & $\mathrm{U} / \mathrm{W}$ \\
\hline 8 & 11 & 1973 & 145 & $15^{\circ} 54.5^{\prime}$ & $-168^{\circ} 27.9^{\prime}$ & 719.7 & 8.4 & 139 & 1.1 & 293 & 9.4 & 136 & $\mathrm{C} / \mathrm{CS}$ \\
\hline 8 & 11 & 1973 & 250 & $15^{\circ} 47.6^{\prime}$ & $-168^{\circ} 21.6^{\prime}$ & 728.8 & 8.1 & 139 & 1.1 & 293 & 9.1 & 136 & $\mathrm{C} / \mathrm{S}$ \\
\hline 8 & 11 & 1973 & 255 & $15^{\circ} 47.1^{\prime}$ & $-168^{\circ} 21.1^{\prime}$ & 729.5 & 8.4 & 139 & 1.1 & 293 & 9.4 & 136 & $\mathrm{C} / \mathrm{S}$ \\
\hline 8 & 11 & 1973 & 310 & $15^{\circ} 45.5^{\prime}$ & $-168^{\circ} 19.7^{\prime}$ & 731.6 & 7.8 & 140 & 1.7 & 297 & 9.4 & 136 & SATL \\
\hline 8 & 11 & 1973 & 510 & $15^{\circ} 33.5^{\prime}$ & $-168^{\circ} \quad 9.3^{\prime}$ & 747.3 & 8.4 & 138 & 1.0 & 298 & 9.4 & 136 & SATL \\
\hline 8 & 11 & 1973 & 80 & $15^{\circ} 15.7^{\prime}$ & $-167^{\circ} 52.8^{\prime}$ & 771.1 & 8.1 & 139 & 1.3 & 299 & 9.4 & 136 & SATL \\
\hline 8 & 11 & 1973 & 1126 & $14^{\circ} 54.7^{\prime}$ & $-167^{\circ} 33.8^{\prime}$ & 799.0 & 8.2 & 141 & 1.4 & 283 & 9.4 & 136 & SATL \\
\hline 8 & 11 & 1973 & 1626 & $14^{\circ} 22.5^{\prime}$ & $-167^{\circ} \quad 7.3^{\prime}$ & 840.2 & 8.0 & 141 & 1.6 & 289 & 9.4 & 136 & SATL \\
\hline 8 & 11 & 1973 & 1820 & $14^{\circ} 10.6^{\prime}$ & $-166^{\circ} 57.4^{\prime}$ & 855.5 & 7.6 & 140 & 1.9 & 298 & 9.4 & 136 & SATL \\
\hline 8 & 11 & 1973 & 1914 & $14^{\circ} \quad 5.3^{\prime}$ & $-166^{\circ} 52.9^{\prime}$ & 862.3 & 7.6 & 137 & 1.9 & 298 & 9.4 & 133 & $\mathrm{C} / \mathrm{C}$ \\
\hline 8 & 11 & 1973 & 1922 & $14^{\circ} 4.6^{\prime}$ & $-166^{\circ} 52.2^{\prime}$ & 863.3. & 8.0 & 141 & 1.9 & 275 & 9.4 & 133 & SATL \\
\hline 8 & 11 & 1973 & 2012 & $13^{\circ} 59.4^{\prime}$ & $-166^{\circ} 47.9^{\prime}$ & 870.0 & 7.7 & 137 & 1.9 & 294 & 9.4 & 133 & SATL \\
\hline 8 & 11 & 1973 & 2246 & $13^{\circ} 44.9^{\prime}$ & $-166^{\circ} 34.2^{\prime}$ & 889.6 & 7.9 & 137 & 1.6 & 293 & 9.4 & 133 & SATL \\
\hline
\end{tabular}


TABLE 1 - Continued

\begin{tabular}{|c|c|c|c|c|c|c|c|c|c|c|c|c|c|c|}
\hline & & & & & & & & & Dri & & & & & \\
\hline DA & MO & YR & Time & Latitude & Longitude & Dist. & Speed & Course & Speed & Hed. & Speed & Course & Comment & Qlty. \\
\hline 8 & 11 & 1973 & 1922 & $14^{\circ} 4.6^{\prime}$ & $-166^{\circ} 52.2^{\prime}$ & 863.3 & 8.0 & 141 & 1.9 & 275 & 9.4 & 133 & SATL & 0.50 \\
\hline 8 & 11 & 1973 & 2012 & $13^{\circ} 59.4^{\prime}$ & $-166^{\circ} 47.9^{\prime}$ & 870.0 & 7.7 & 137 & 1.9 & 294 & 9.4 & 133 & SATL & 0.25 \\
\hline 8 & 11 & 1973 & 2246 & $13^{\circ} 44.9^{\prime}$ & $-166^{\circ} 34.2^{\prime}$ & 889.6 & 7.9 & 137 & 1.6 & 293 & 9.4 & 133 & SATL & 0.25 \\
\hline 8 & 11 & 1973 & 2312 & $13^{\circ} 42.4^{\prime}$ & $-166^{\circ} 31.8^{\prime}$ & 893.1 & 7.9 & 137 & 1.7 & 293 & 9.4 & 133 & SATL & 1.00 \\
\hline 9 & 11 & 1973 & 00 & $13^{\circ} 37.8^{\prime}$ & $-166^{\circ} 27.4^{\prime}$ & 899.4 & 7.9 & 137 & 1.7 & 293 & 9.4 & 133 & & \\
\hline 9 & 11 & 1973 & 056 & $13^{\circ} 32.4^{\prime}$ & $-166^{\circ} 22.3^{\prime}$ & 906.7 & 8.0 & 138 & 1.6 & 285 & 9.4 & 133 & SATL & 1.50 \\
\hline 9 & 11 & 1973 & 324 & $13^{\circ} 17.6^{\prime}$ & $-166^{\circ} 8.7^{\prime}$ & 926.5 & 7.9 & 139 & 1.8 & 285 & 9.4 & 133 & SATL & 0.50 \\
\hline 9 & 11 & 1973 & 76 & $12^{\circ} 55.6^{\prime}$ & $-165^{\circ} 49.0^{\prime}$ & 955.7 & 7.9 & 137 & 1.6 & 294 & 9.4 & 133 & SATL & 0.50 \\
\hline 9 & 11 & 1973 & 720 & $12^{\circ} 54.3^{\prime}$ & $-165^{\circ} 47.7^{\prime}$ & 957.6 & 7.3 & 137 & 1.6 & 294 & 8.8 & 133 & $\mathrm{C} / \mathrm{S}$ & \\
\hline 9 & 11 & 1973 & 840 & $12^{\circ} 47.1^{\prime}$ & $-165^{\circ} 40.9^{\prime}$ & 967.3 & 7.8 & 138 & 1.2 & 276 & 8.8 & 133 & SATL & 0.50 \\
\hline 9 & 11 & 1973 & 930 & $12^{\circ} 42.2^{\prime}$ & $-165^{\circ} 36.5^{\prime}$ & 973.8 & 7.9 & 142 & 1.2 & 276 & 8.8 & 136 & $\mathrm{C} / \mathrm{C}$ & \\
\hline 9 & 11 & 1973 & 1038 & $12^{\circ} 35.2^{\prime}$ & $-165^{\circ} 30.8^{\prime}$ & 982.8 & 8.4 & 140 & 0.7 & 260 & 8.8 & 136 & SATL & 0.25 \\
\hline 9 & 11 & 1973 & 134 & $12^{\circ} 19.5^{\prime}$ & $-165^{\circ} 17.2^{\prime}$ & 1003.3 & 8.9 & 136 & 0.2 & 116 & 8.8 & 136 & SATL & 1.00 \\
\hline 9 & 11 & 1973 & 1450 & $12^{\circ} 8.2^{\prime}$ & $-165^{\circ} 5.9^{\prime}$ & 1019.1 & 8.8 & 137 & 0.2 & 215 & 8.8 & 136 & SATL & 1.50 \\
\hline 9 & 11 & 1973 & 1730 & $11^{\circ} 50.9^{\prime}$ & $-164^{\circ} 49.5^{\prime}$ & 1042.7 & 9.1 & 137 & 0.2 & 215 & 9.1 & 136 & $\mathrm{C} / \mathrm{S}$ & \\
\hline 9 & 11 & 1973 & 1732 & $11^{\circ} 50.7^{\prime}$ & $-164^{\circ} 49.3^{\prime}$ & 1043.0 & 9.5 & 138 & 0.5 & 185 & 9.1 & 136 & SATL & 1.00 \\
\hline 9 & 11 & 1973 & 1830 & $11^{\circ} 43.8^{\prime}$ & $-164^{\circ} 43.1^{\prime}$ & 1052.2 & 9.5 & 141 & 0.5 & 185 & 9.1 & 139 & $\mathrm{C} / \mathrm{C}$ & \\
\hline 9 & 11 & 1973 & 1832 & $11^{\circ} 43.6^{\prime}$ & $-164^{\circ} 42.9^{\prime}$ & 1052.5 & 9.2 & 144 & 0.7 & 220 & 9.1 & 139 & SATL & 0.00 \\
\hline 9 & 11 & 1973 & 1918 & $11^{\circ} 37.9^{\prime}$ & $-164^{\circ} 38.6^{\prime}$ & 1059.6 & 9.0 & 142 & 0.4 & 274 & 9.1 & 139 & SATL & 1.00 \\
\hline 9 & 11 & 1973 & 2016 & $11^{\circ} 31.1^{\prime}$ & $-164^{\circ} 33.1^{\prime}$ & 1068.2 & 8.9 & 143 & 0.7 & 248 & 9.1 & 139 & SATL & 1.00 \\
\hline 9 & 11 & 1973 & 2050 & $11^{\circ} 27.1^{\prime}$ & $-164^{\circ} 30.0^{\prime}$ & 1073.3 & 6.9 & 144 & 0.7 & 248 & 7.0 & 139 & $\mathrm{C} / \mathrm{S}$ & \\
\hline 9 & 11 & 1973 & 2055 & $11^{\circ} 26.6^{\prime}$ & $-164^{\circ} 29.7^{\prime}$ & 1073.8 & 5.9 & 145 & 0.7 & 248 & 6.1 & 139 & $\mathrm{C} / \mathrm{S}$ & \\
\hline 9 & 11 & 1973 & 2150 & $11^{\circ} 22.1^{\prime}$ & $-164^{\circ} 26.5^{\prime}$ & 1079.3 & 7.7 & 144 & 0.7 & 248 & 7.9 & 139 & $\mathrm{C} / \mathrm{S}$ & \\
\hline 9 & 11 & 1973 & 2155 & $11^{\circ} 21.6^{\prime}$ & $-164^{\circ} 26.1^{\prime}$ & 1079.9 & 7.5 & 144 & 0.7 & 248 & 7.7 & 139 & $\mathrm{C} / \mathrm{S}$ & \\
\hline 9 & 11 & 1973 & 2222 & $11^{\circ} 18.9^{\prime}$ & $-164^{\circ} 24.1^{\prime}$ & 1083.3 & 7.2 & 148 & 1.3 & 258 & 7.7 & 139 & SATL & 0.50 \\
\hline 9 & 11 & 1973 & 2245 & $11^{\circ} 16.6^{\prime}$ & $-164^{\circ} 22.6^{\prime}$ & 1086.0 & 6.5 & 149 & 1.3 & 258 & 7.0 & 139 & $\mathrm{C} / \mathrm{S}$ & \\
\hline 9 & 11 & 1973 & 2247 & $11^{\circ} 16.4^{\prime}$ & $-164^{\circ} 22.5^{\prime}$ & 1086.3 & 8.8 & 146 & 1.3 & 258 & 9.4 & 139 & $\mathrm{C} / \mathrm{S}$ & \\
\hline 10 & 11 & 1973 & 00 & $11^{\circ} 7.4^{\prime}$ & $-164^{\circ} 16.4^{\prime}$ & 1097.0 & 8.8 & 146 & 1.3 & 258 & 9.4 & 139 & & \\
\hline 10 & 11 & 1973 & 06 & $11^{\circ} 6.7^{\prime}$ & $-164^{\circ} 15.9^{\prime}$ & 1097.9 & 9.1 & 143 & 0.7 & 253 & 9.4 & 139 & SATL & 1.00 \\
\hline 10 & 11 & 1973 & 040 & $11^{\circ} 2.6^{\prime}$ & $-164^{\circ} 12.7^{\prime}$ & 1103.1 & 9.1 & 139 & 0.7 & 253 & 9.4 & 135 & $\mathrm{C} / \mathrm{C}$ & \\
\hline 10 & 11 & 1973 & 129 & $10^{\circ} 57.0^{\prime}$ & $-164^{\circ} \quad 7.8^{\prime}$ & 1110.5 & 7.3 & 140 & 0.7 & 253 & 7.6 & 135 & $\mathrm{C} / \mathrm{S}$ & \\
\hline 10 & 11 & 1973 & 236 & $10^{\circ} 50.7^{\prime}$ & $-164^{\circ} \quad 2.5^{\prime}$ & 1118.6 & 7.8 & 142 & 0.9 & 218 & 7.6 & 135 & SATL & 0.50 \\
\hline 10 & 11 & 1973 & 315 & $10^{\circ} 46.7^{\prime}$ & $-163^{\circ} 59.3^{\prime}$ & 1123.7 & 4.2 & 148 & 0.9 & 218 & 4.0 & 135 & $\mathrm{C} / \mathrm{S}$ & \\
\hline 10 & 11 & 1973 & 335 & $10^{\circ} 45.5^{\prime}$ & $-163^{\circ} 58.6^{\prime}$ & 1125.1 & 4.2 & 145 & 0.9 & 218 & 4.0 & 132 & $\mathrm{C} / \mathrm{C}$ & \\
\hline 10 & 11 & 1973 & 345 & $10^{\circ} 45.0^{\prime}$ & $-163^{\circ} 58.1^{\prime}$ & 1125.8 & 7.7 & 139 & 0.9 & 218 & 7.6 & 132 & $\mathrm{C} / \mathrm{S}$ & \\
\hline 10 & 11 & 1973 & 436 & $10^{\circ} 40.0^{\prime}$ & $-163^{\circ} 53.8^{\prime}$ & 1132.4 & 5.9 & 141 & 0.9 & 218 & 5.8 & 132 & $\mathrm{C} / \mathrm{S}$ & \\
\hline 10 & 11 & 1973 & 449 & $10^{\circ} 39.0^{\prime}$ & $-163^{\circ} 52.9^{\prime}$ & 1133.6 & 7.7 & 139 & 0.9 & 218 & 7.6 & 132 & $\mathrm{C} / \mathrm{S}$ & \\
\hline 10 & 11 & 1973 & 516 & $10^{\circ} 36.4^{\prime}$ & $-163^{\circ} 50.6^{\prime}$ & 1137.1 & 8.2 & 137 & 1.0 & 185 & 7.6 & 132 & SATL & 1.00 \\
\hline 10 & 11 & 1973 & 72 & $10^{\circ} 25.7^{\prime}$ & $-163^{\circ} 40.6^{\prime}$ & 1151.7 & 8.1 & 137 & 0.9 & 189 & 7.6 & 132 & SATL & 1.00 \\
\hline 10 & 11 & 1973 & 930 & $10^{\circ} 10.9^{\prime}$ & $-163^{\circ} 26.8^{\prime}$ & 1171.7 & 8.2 & 138 & 1.1 & 189 & 7.6 & 132 & SATL & 1.50 \\
\hline 10 & 11 & 1973 & 1136 & $9^{\circ} 58.0^{\prime}$ & $-163^{\circ} 15.1^{\prime}$ & 1189.0 & 8.3 & 140 & 1.3 & 193 & 7.6 & 132 & SATL & 0.50 \\
\hline 10 & 11 & 1973 & 126 & $9^{\circ} 54.8^{\prime}$ & $-163^{\circ} 12.4^{\prime}$ & 1193.2 & 8.4 & 146 & 1.3 & 193 & 7.6 & 139 & $\mathrm{C} / \mathrm{C}$ & \\
\hline 10 & 11 & 1973 & 1230 & $9^{\circ} 52.0^{\prime}$ & $-163^{\circ} 10.5^{\prime}$ & 1196.5 & 8.3 & 140 & 1.3 & 193 & 7.6 & 132 & $\mathrm{C} / \mathrm{C}$ & \\
\hline 10 & 11 & 1973 & 1410 & $9^{\circ} 41.5^{\prime}$ & $-163^{\circ} 1.4^{\prime}$ & 1210.4 & 8.4 & 135 & 0.9 & 160 & 7.6 & 132 & SATL & 1.50 \\
\hline 10 & 11 & 1973 & 1830 & $9^{\circ} 15.9^{\prime}$ & $-162^{\circ} 35.3^{\prime}$ & 1246.7 & 8.5 & 126 & 1.2 & 82 & 7.6 & 132 & SATL & 1.00 \\
\hline 10 & 11 & 1973 & 1920 & $9^{\circ} 11.8^{\prime}$ & $-162^{\circ} 29.5^{\prime}$ & 1253.7 & 8.3 & 128 & 0.8 & 92 & 7.6 & 132 & SATL & 0.50 \\
\hline 10 & 11 & 1973 & 2010 & $9^{\circ} 7.5^{\prime}$ & $-162^{\circ} 24.0^{\prime}$ & 1260.6 & 8.2 & 132 & 0.8 & 92 & 7.6 & 136 & $\mathrm{C} / \mathrm{C}$ & \\
\hline 10 & 11 & 1973 & 2250 & $8^{\circ} 52.9^{\prime}$ & $-162^{\circ} 7.5^{\prime}$ & 1282.5 & 8.3 & 130 & 1.1 & 85 & 7.6 & 136 & SATL & 0.25 \\
\hline 10 & 11 & 1973 & 233 & $8^{\circ} 51.7^{\prime}$ & $-162^{\circ} 6.1^{\prime}$ & 1284.3 & 8.3 & 134 & 1.1 & 85 & 7.6 & 140 & $\mathrm{C} / \mathrm{C}$ & \\
\hline 10 & 11 & 1973 & 2318 & $8^{\circ} 50.3^{\prime}$ & $-162^{\circ} \quad 4.6^{\prime}$ & 1286.4 & 8.2 & 134 & 1.0 & 85 & 7.6 & 140 & SATL & 0.25 \\
\hline 11 & 11 & 1973 & 00 & $8^{\circ} 46.3^{\prime}$ & $-162^{\circ} 0.4^{\prime}$ & 1292.1 & 8.2 & 134 & 1.0 & 85 & 7.6 & 140 & & \\
\hline 11 & 11 & 1973 & 18 & $8^{\circ} 39.8^{\prime}$ & $-161^{\circ} 53.7^{\prime}$ & 1301.4 & 8.3 & 132 & 1.3 & 76 & 7.6 & 140 & SATL & 0.50 \\
\hline 11 & 11 & 1973 & 132 & $8^{\circ} 37.6^{\prime}$ & $-161^{\circ} 51.2^{\prime}$ & 1304.7 & 8.0 & 130 & 1.4 & 60 & 7.6 & 140 & SATL & 1.50 \\
\hline 11 & 11 & 1973 & 148 & $8^{\circ} 36.2^{\prime}$ & $-161^{\circ} 49.6^{\prime}$ & 1306.9 & 7.8 & 138 & 1.4 & 60 & 7.6 & 148 & $\mathrm{C} / \mathrm{C}$ & \\
\hline 11 & 11 & 1973 & 428 & $8^{\circ} 20.9^{\prime}$ & $-161^{\circ} 35.4^{\prime}$ & 1327.6 & 8.1 & 138 & 1.4 & 74 & 7.6 & 148 & SATL & 0.25 \\
\hline 11 & 11 & 1973 & 842 & $7^{\circ} 55.3^{\prime}$ & $-161^{\circ} 12.4^{\prime}$ & 1361.9 & 8.3 & 139 & 1.4 & 83 & 7.6 & 148 & SATL & 0.00 \\
\hline 11 & 11 & 1973 & 920 & $7^{\circ} 51.3^{\prime}$ & $-161^{\circ} 8.9^{\prime}$ & 1367.2 & 8.2 & 143 & 1.4 & 83 & 7.6 & 152 & $\mathrm{C} / \mathrm{C}$ & \\
\hline 11 & 11 & 1973 & 1050 & $7^{\circ} 41.5^{\prime}$ & $-161^{\circ} \quad 1.4^{\prime}$ & 1379.5 & 8.2 & 148 & 0.8 & 112 & 7.6 & 152 & SATL & 1.00 \\
\hline 11 & 11 & 1973 & 1236 & $7^{\circ} 29.1^{\prime}$ & $-160^{\circ} 53.7^{\prime}$ & 1394.0 & 7.8 & 142 & 1.3 & 64 & 7.6 & 152 & SATL & 1.00 \\
\hline 11 & 11 & 1973 & 1351 & $7^{\circ} 21.4^{\prime}$ & $-160^{\circ} 47.7^{\prime}$ & 1403.7 & 7.9 & 136 & 1.3 & 64 & 7.6 & 145 & $\mathrm{C} / \mathrm{C}$ & \\
\hline 11 & 11 & 1973 & 1742 & $6^{\circ} 59.6^{\prime}$ & $-160^{\circ} 26.3^{\prime}$ & 1434.2 & 8.1 & 134 & 1.6 & 68 & 7.6 & 145 & SATL & 1.00 \\
\hline 11 & 11 & 1973 & 187 & $6^{\circ} 57.2^{\prime}$ & $-160^{\circ} 23.8^{\prime}$ & 1437.6 & 8.0 & 137 & 1.6 & 68 & 7.6 & 148 & $\mathrm{C} / \mathrm{C}$ & \\
\hline 11 & 11 & 1973 & 1828 & $6^{\circ} 55.2^{\prime}$ & $-160^{\circ} 21.9^{\prime}$ & 1440.4 & 7.0 & 136 & 1.7 & 31 & 7.6 & 148 & SATL & 0.25 \\
\hline 11 & 11 & 1973 & 1848 & $6^{\circ} 53.5^{\prime}$ & $-160^{\circ} 20.1^{\prime}$ & 1442.7 & 5.8 & 133 & 1.7 & 31 & 6.4 & 148 & $\mathrm{C} / \mathrm{S}$ & \\
\hline 11 & 11 & 1973 & 1858 & $6^{\circ} 52.9^{\prime}$ & $-160^{\circ} 19.5^{\prime}$ & 1443.7 & 7.0 & 136 & 1.7 & 31 & 7.6 & 148 & $\mathrm{C} / \mathrm{S}$ & \\
\hline 11 & 11 & 1973 & 1930 & $6^{\circ} 50.2^{\prime}$ & $-160^{\circ} 16.9^{\prime}$ & 1447.5 & 7.5 & 134 & 1.8 & 49 & 7.6 & 148 & SATL & 1.00 \\
\hline 11 & 11 & 1973 & 200 & $6^{\circ} 47.6^{\prime}$ & $-160^{\circ} 14.2^{\prime}$ & 1451.2 & 7.4 & 138 & 1.8 & 49 & 7.6 & 152 & $\mathrm{C} / \mathrm{C}$ & \\
\hline 11 & 11 & 1973 & 2156 & $6^{\circ} 36.9^{\prime}$ & $-160^{\circ} 4.6^{\prime}$ & 1465.5 & 7.2 & 139 & 1.7 & 43 & 7.6 & 152 & SATL & 0.50 \\
\hline 11 & 11 & 1973 & 2230 & $6^{\circ} 33.8^{\prime}$ & $-160^{\circ} \quad 1.9^{\prime}$ & 1469.6 & 7.2 & 138 & 1.8 & 43 & 7.6 & 152 & SATL & 1.50 \\
\hline 12 & 11 & $197 \hat{3}$ & 00 & $6^{\circ} 25.7^{\prime}$ & $-159^{\circ} 54.6^{\prime}$ & 1480.4 & 7.2 & 138 & 1.8 & 43 & 7.6 & 152 & & \\
\hline 12 & 11 & 1973 & 014 & $6^{\circ} 24.5^{\prime}$ & $-159^{\circ} 53.5^{\prime}$ & 1482.1 & 6.7 & 144 & 1.4 & 15 & 7.6 & 152 & SATL & 1.50 \\
\hline 12 & 11 & 1973 & 048 & $6^{\circ} 21.4^{\prime}$ & $-159^{\circ} 51.3^{\prime}$ & 1485.9 & 6.5 & 159 & 1.4 & 15 & 7.6 & 165 & $\mathrm{C} / \mathrm{C}$ & \\
\hline 12 & 11 & 1973 & 214 & $6^{\circ} 12.8^{\prime}$ & $-159^{\circ} 47.9^{\prime}$ & 1495.2 & 6.3 & 165 & 1.3 & 345 & 7.6 & 165 & SATL & 0.50 \\
\hline 12 & 11 & 1973 & 235 & $6^{\circ} 10.7^{\prime}$ & $-159^{\circ} 47.3^{\prime}$ & 1497.4 & 6.4 & 153 & 1.3 & 345 & 7.6 & 155 & $\mathrm{C} / \mathrm{C}$ & \\
\hline 12 & 11 & 1973 & 358 & $6^{\circ} 2.8^{\prime}$ & $-159^{\circ} 43.3^{\prime}$ & 1506.2 & 6.3 & 154 & 1.3 & 339 & 7.6 & 155 & SATL & 1.00 \\
\hline 12 & 11 & 1973 & 522 & $5^{\circ} 54.8^{\prime}$ & $-159^{\circ} 39.4^{\prime}$ & 1515.1 & 6.5 & 157 & 1.2 & 324 & 7.6 & 155 & SATL & 1.50 \\
\hline 12 & 11 & 1973 & 555 & $5^{\circ} 51.5^{\prime}$ & $-159^{\circ} 38.0^{\prime}$ & 1518.6 & 6.4 & 149 & 1.2 & 324 & 7.6 & 148 & $\mathrm{C} / \mathrm{C}$ & \\
\hline 12 & 11 & 1973 & 64 & $5^{\circ} 50.7^{\prime}$ & $-159^{\circ} 37.5^{\prime}$ & 1519.6 & 6.5 & 151 & 1.2 & 313 & 7.6 & 148 & SATL & 0.50 \\
\hline 12 & 11 & 1973 & 712 & $5^{\circ} 44.3^{\prime}$ & $-159^{\circ} 33.9^{\prime}$ & 1526.9 & 6.8 & 151 & 0.9 & 303 & 7.6 & 148 & SATL & 1.00 \\
\hline 12 & 11 & 1973 & 745 & $5^{\circ} 41.0^{\prime}$ & $-159^{\circ} 32.1^{\prime}$ & 1530.7 & 6.8 & 148 & 0.9 & 303 & 7.6 & 145 & $\mathrm{C} / \mathrm{C}$ & \\
\hline 12 & 11 & 1973 & 936 & $5^{\circ} 30.4^{\prime}$ & $-159^{\circ} 25.4^{\prime}$ & 1543.2 & 7.1 & 155 & 1.4 & 263 & 7.6 & 145 & SATL & 0.25 \\
\hline 12 & 11 & 1973 & 106 & $5^{\circ} 27.2^{\prime}$ & $-159^{\circ} 23.9^{\prime}$ & 1546.8 & 7.0 & 149 & 0.8 & 287 & 7.6 & 145 & SATL & 0.00 \\
\hline
\end{tabular}


TABLE 1 - Continued

\begin{tabular}{|c|c|c|c|c|c|c|c|c|c|c|c|c|c|c|}
\hline & & & & & & & & ual & Drit & & & & & \\
\hline DA & MO & YR & Time & Latitude & Longitude & Dist. & Speed & Course & Speed & Hed. & Speed & Course & Comment & Qlty. \\
\hline 12 & 11 & 1973 & 1018 & $5^{\circ} 26.0^{\prime}$ & $-159^{\circ} 23.2^{\prime}$ & 1548.2 & 6.9 & 133 & 0.8 & 287 & 7.6 & 130 & $\mathrm{C} / \mathrm{C}$ & \\
\hline 12 & 11 & 1973 & 1148 & $5^{\circ} 19.0^{\prime}$ & $-159^{\circ} 15.5^{\prime}$ & 1558.5 & 6.6 & 138 & 1.4 & 270 & 7.6 & 130 & SATL & 1.50 \\
\hline 12 & 11 & 1973 & 1217 & $5^{\circ} 16.6^{\prime}$ & $-159^{\circ} 13.3^{\prime}$ & 1561.7 & 6.7 & 143 & 1.4 & 270 & 7.6 & 135 & $\mathrm{C} / \mathrm{C}$ & \\
\hline 12 & 11 & 1973 & 1526 & $4^{\circ} 59.7^{\prime}$ & $\begin{array}{lll}-159^{\circ} & 0.7^{\prime}\end{array}$ & 1582.8 & 6.8 & 146 & 1.6 & 259 & 7.6 & 135 & SATL & 0.50 \\
\hline 12 & 11 & 1973 & 160 & $4^{\circ} 56.5^{\prime}$ & $-158^{\circ} 58.5^{\prime}$ & 1586.7 & 6.6 & 137 & 1.6 & 259 & 7.6 & 127 & $\mathrm{C} / \mathrm{C}$ & \\
\hline 12 & 11 & 1973 & 1654 & $4^{\circ} 52.1^{\prime}$ & $-158^{\circ} 54.5^{\prime}$ & 1592.6 & 6.8 & 137 & 1.5 & 256 & 7.6 & 127 & SATL & 1.00 \\
\hline 12 & 11 & 1973 & 1840 & $4^{\circ} 43.4^{\prime}$ & $-158^{\circ} 46.3^{\prime}$ & 1604.6 & 6.6 & 130 & 1.1 & 287 & 7.6 & 127 & SATL & 1.00 \\
\hline 12 & 11 & 1973 & 1918 & $4^{\circ} 40.7^{\prime}$ & $-158^{\circ} 43.1^{\prime}$ & 1608.7 & 6.6 & 130 & 1.0 & 288 & 7.6 & 127 & SATL & 0.50 \\
\hline 12 & 11 & 1973 & 214 & $4^{\circ} 33.2^{\prime}$ & $-158^{\circ} 34.1^{\prime}$ & 1620.4 & 6.7 & 129 & 0.9 & 289 & 7.6 & 127 & SATL & 0.25 \\
\hline 12 & 11 & 1973 & 2127 & $4^{\circ} 31.6^{\prime}$ & $-158^{\circ} 32.1^{\prime}$ & 1623.0 & 6.7 & 134 & 0.9 & 289 & 7.6 & 131 & $\mathrm{C} / \mathrm{C}$ & \\
\hline 12 & 11 & 1973 & 2142 & $4^{\circ} 30.4^{\prime}$ & $-158^{\circ} 30.9^{\prime}$ & 1624.7 & 6.8 & 135 & 1.0 & 282 & 7.6 & 131 & SATL & 0.25 \\
\hline 12 & 11 & 1973 & 2147 & $4^{\circ} 30.0^{\prime}$ & $-158^{\circ} 30.5^{\prime}$ & 1625.3 & 6.8 & 138 & 1.0 & 282 & 7.6 & 134 & $\mathrm{C} / \mathrm{C}$ & \\
\hline 12 & 11 & 1973 & 2251 & $4^{\circ} 24.6^{\prime}$ & $-158^{\circ} 25.7^{\prime}$ & 1632.5 & 6.8 & 137 & 1.0 & 282 & 7.6 & 133 & $\mathrm{C} / \mathrm{C}$ & \\
\hline 12 & 11 & 1973 & 2328 & $4^{\circ} 21.5^{\prime}$ & $-158^{\circ} 22.8^{\prime}$ & 1636.7 & 6.8 & 144 & 1.6 & 259 & 7.6 & 133 & SATL & 1.00 \\
\hline 13 & 11 & 1973 & 00 & $4^{\circ} 18.6^{\prime}$ & $-158^{\circ} 20.6^{\prime}$ & 1640.3 & 6.8 & 144 & 1.6 & 259 & 7.6 & 133 & & \\
\hline 13 & 11 & 1973 & 028 & $4^{\circ} 16.0^{\prime}$ & $-158^{\circ} 18.8^{\prime}$ & 1643.5 & 9.1 & 247 & 1.6 & 259 & 7.6 & 245 & $\mathrm{C} / \mathrm{C}$ & \\
\hline 13 & 11 & 1973 & 16 & $4^{\circ} 13.8^{\prime}$ & $-158^{\circ} 24.1^{\prime}$ & 1649.3 & 9.5 & 245 & 1.9 & 243 & 7.6 & 245 & SATL & 1.00 \\
\hline 13 & 11 & 1973 & 145 & $4^{\circ} 11.2^{\prime}$ & $-158^{\circ} 29.7^{\prime}$ & 1655.5 & 8.0 & 245 & 1.9 & 243 & 6.1 & 245 & $\mathrm{C} / \mathrm{S}$ & \\
\hline 13 & 11 & 1973 & 20 & $4^{\circ} 10.3^{\prime}$ & $-158^{\circ} 31.5^{\prime}$ & 1657.5 & 6.1 & 245 & 0.0 & 0 & 6.1 & 245 & S315 & 1.50 \\
\hline 13 & 11 & 1973 & 20 & $4^{\circ} 10.3^{\prime}$ & $-158^{\circ} 31.5^{\prime}$ & 1657.5 & 0.0 & 0 & 0.0 & 0 & 0.0 & 500 & STOP & \\
\hline 19 & 11 & 1973 & 152 & $4^{\circ} 10.3^{\prime}$ & $-158^{\circ} 31.5^{\prime}$ & 1657.5 & 2.0 & 283 & 2.0 & 283 & 0.0 & 500 & DEP & 1.50 \\
\hline 19 & 11 & 1973 & 152 & $4^{\circ} 10.3^{\prime}$ & $-158^{\circ} 31.5^{\prime}$ & 1657.5 & 9.7 & 257 & 2.0 & 283 & 7.9 & 250 & $\mathrm{U} / \mathrm{W}$ & \\
\hline 19 & 11 & 1973 & 155 & $4^{\circ} 10.2^{\prime}$ & $-158^{\circ} 32.0^{\prime}$ & 1657.9 & 8.8 & 257 & 2.0 & 283 & 7.0 & 250 & $\mathrm{C} / \mathrm{s}$ & \\
\hline 19 & 11 & 1973 & 1634 & $4^{\circ} 7.3^{\prime}$ & $-158^{\circ} 44.7^{\prime}$ & 1671.0 & 8.6 & 257 & 1.9 & 287 & 7.0 & 250 & SATL & 1.00 \\
\hline 19 & 11 & 1973 & 1840 & $4^{\circ} 3.4^{\prime}$ & $-159^{\circ} \quad 2.3^{\prime}$ & 1688.9 & 9.1 & 261 & 2.6 & 292 & 7.0 & 250 & SATL & 0.50 \\
\hline 19 & 11 & 1973 & 196 & $4^{\circ} \quad 2.8^{\prime}$ & $-159^{\circ} 6.2^{\prime}$ & 1692.9 & 9.2 & 264 & 2.6 & 292 & 7.0 & 254 & $\mathrm{C} / \mathrm{C}$ & \\
\hline 19 & 11 & 1973 & 1944 & $4^{\circ} \quad 2.2^{\prime}$ & $-159^{\circ} 12.0^{\prime}$ & 1698.7 & 9.1 & 261 & 2.6 & 292 & 7.0 & 250 & $\mathrm{C} / \mathrm{C}$ & \\
\hline 19 & 11 & 1973 & 1950 & $4^{\circ} \quad 2.1^{\prime}$ & $-159^{\circ} 12.9^{\prime}$ & 1699.6 & 6.2 & 267 & 2.6 & 292 & 4.0 & 250 & $\mathrm{C} / \mathrm{S}$ & \\
\hline 19 & 11 & 1973 & 1951 & $4^{\circ} 2.1^{\prime}$ & $-159^{\circ} 13.0^{\prime}$ & 1699.7 & 6.1 & 263 & 2.6 & 292 & 4.0 & 245 & $\mathrm{C} / \mathrm{C}$ & \\
\hline 19 & 11 & 1973 & 1954 & $4^{\circ} \quad 2.0^{\prime}$ & $-159^{\circ} 13.3^{\prime}$ & 1700.0 & 9.6 & 257 & 2.6 & 292 & 7.6 & 245 & $\mathrm{C} / \mathrm{s}$ & \\
\hline 19 & 11 & 1973 & 2022 & $4^{\circ} \quad 1.0^{\prime}$ & $-159^{\circ} 17.7^{\prime}$ & 1704.5 & 9.4 & 253 & 2.6 & 292 & 7.6 & 240 & $\mathrm{C} / \mathrm{C}$ & \\
\hline 19 & 11 & 1973 & 2024 & $\begin{array}{ll}4^{\circ} & 0.9^{\prime}\end{array}$ & $-159^{\circ} 18.0^{\prime}$ & 1704.8 & 8.6 & 245 & 1.3 & 280 & 7.6 & 240 & SATL & 1.00 \\
\hline 19 & 11 & 1973 & 2027 & $\begin{array}{ll}4^{\circ} & 0.7^{\prime}\end{array}$ & $-159^{\circ} 18.4^{\prime}$ & 1705.3 & 9.8 & 245 & 1.3 & 280 & 8.8 & 240 & $\mathrm{C} / \mathrm{S}$ & \\
\hline 19 & 11 & 1973 & 2034 & $4^{\circ} \quad 0.2^{\prime}$ & $-159^{\circ} 19.4^{\prime}$ & 1706.4 & 9.8 & 240 & 1.3 & 280 & 8.8 & 235 & $\mathrm{C} / \mathrm{C}$ & \\
\hline 19 & 11 & 1973 & 2048 & $3^{\circ} 59.1^{\prime}$ & $-159^{\circ} 21.4^{\prime}$ & 1708.7 & 8.0 & 242 & 1.3 & 280 & 7.0 & 235 & $\mathrm{C} / \mathrm{S}$ & \\
\hline 19 & 11 & 1973 & 2050 & $3^{\circ} 59.0^{\prime}$ & $-159^{\circ} 21.6^{\prime}$ & 1708.9 & 7.8 & 233 & 1.3 & 280 & 7.0 & 225 & $\mathrm{C} / \mathrm{C}$ & \\
\hline 19 & 11 & 1973 & 213 & $3^{\circ} 58.0^{\prime}$ & $-159^{\circ} 23.0^{\prime}$ & 1710.6 & 6.5 & 225 & 1.3 & 280 & 5.8 & 215 & $\mathrm{C} / \mathrm{CS}$ & \\
\hline 19 & 11 & 1973 & 217 & $3^{\circ} 57.7^{\prime}$ & $-159^{\circ} 23.3^{\prime}$ & 1711.1 & 6.4 & 221 & 1.3 & 280 & 5.8 & 210 & $\mathrm{C} / \mathrm{C}$ & \\
\hline 19 & 11 & 1973 & 2120 & $3^{\circ} 56.6^{\prime}$ & $-159^{\circ} 24.2^{\prime}$ & 1712.4 & 5.9 & 202 & 1.3 & 280 & 5.8 & 190 & $\mathrm{C} / \mathrm{C}$ & \\
\hline 19 & 11 & 1973 & 2145 & $3^{\circ} 54.3^{\prime}$ & $-159^{\circ} 25.2^{\prime}$ & 1714.9 & 1.3 & 280 & 1.3 & 280 & 0.0 & 500 & STOP & \\
\hline 19 & 11 & 1973 & 2347 & $3^{\circ} 54.8^{\prime}$ & $-159^{\circ} 27.7^{\prime}$ & 1717.5 & 5.5 & 183 & 1.3 & 280 & 5.8 & 170 & $\mathrm{U} / \mathrm{W}$ & \\
\hline 19 & 11 & 1973 & 2356 & $3^{\circ} 53.9^{\prime}$ & $-159^{\circ} 27.8^{\prime}$ & 1718.3 & 5.3 & 172 & 1.3 & 280 & 5.8 & 160 & $\mathrm{C} / \mathrm{C}$ & \\
\hline 20 & 11 & 1973 & 00 & $3^{\circ} 53.6^{\prime}$ & $-159^{\circ} 27.7^{\prime}$ & 1718.7 & 5.3 & 172 & 1.3 & 280 & 5.8 & 160 & & \\
\hline 20 & 11 & 1973 & 05 & $3^{\circ} 53.2^{\prime}$ & $-159^{\circ} 27.7^{\prime}$ & 1719.1 & 6.5 & 170 & 1.3 & 280 & 7.0 & 160 & $\mathrm{C} / \mathrm{S}$ & \\
\hline 20 & 11 & 1973 & 011 & $3^{\circ} 52.5^{\prime}$ & $-159^{\circ} 27.5^{\prime}$ & 1719.8 & 7.3 & 210 & 1.3 & 280 & 7.0 & 200 & $\mathrm{C} / \mathrm{C}$ & \\
\hline 20 & 11 & 1973 & 225 & $3^{\circ} 38.3^{\prime}$ & $-159^{\circ} 35.7^{\prime}$ & 1736.2 & 8.2 & 209 & 1.3 & 280 & 7.9 & 200 & $\mathrm{C} / \mathrm{s}$ & \\
\hline 20 & 11 & 1973 & 228 & $3^{\circ} 38.0^{\prime}$ & $-159^{\circ} 35.9^{\prime}$ & 1736.6 & 9.1 & 208 & 1.3 & 280 & 8.8 & 200 & $\mathrm{C} / \mathrm{S}$ & \\
\hline 20 & 11 & 1973 & 31 & $3^{\circ} 33.5^{\prime}$ & $-159^{\circ} 38.3^{\prime}$ & 1741.6 & 9.0 & 202 & 1.3 & 280 & 8.8 & 194 & $\mathrm{C} / \mathrm{C}$ & \\
\hline 20 & 11 & 1973 & 412 & $3^{\circ} 23.7^{\prime}$ & $-159^{\circ} 42.3^{\prime}$ & 1752.2 & 8.9 & 211 & 2.6 & 290 & 8.8 & 194 & SATL & 1.50 \\
\hline 20 & 11 & 1973 & 452 & $3^{\circ} 18.6^{\prime}$ & $-159^{\circ} 45.3^{\prime}$ & 1758.2 & 8.3 & 197 & 2.6 & 290 & 8.8 & 180 & $\mathrm{C} / \mathrm{C}$ & \\
\hline 20 & 11 & 1973 & 548 & $3^{\circ} 11.2^{\prime}$ & $-159^{\circ} 47.6^{\prime}$ & 1765.9 & 7.7 & 195 & 2.5 & 304 & 8.8 & 180 & SATL & 0.50 \\
\hline 20 & 11 & 1973 & 86 & $2^{\circ} 54.1^{\prime}$ & $-159^{\circ} 52.3^{\prime}$ & 1783.6 & 7.3 & 196 & 2.7 & 311 & 8.8 & 180 & SATL & 1.00 \\
\hline 20 & 11 & 1973 & 841 & $2^{\circ} 50.0^{\prime}$ & $-159^{\circ} 53.5^{\prime}$ & 1787.9 & 6.5 & 172 & 2.7 & 311 & 8.8 & 160 & $\mathrm{C} / \mathrm{C}$ & \\
\hline 20 & 11 & 1973 & 1042 & $2^{\circ} 37.0^{\prime}$ & $-159^{\circ} 51.6^{\prime}$ & 1801.0 & 6.7 & 173 & 2.8 & 307 & 8.8 & 160 & SATL & 0.50 \\
\hline 20 & 11 & 1973 & 1056 & $2^{\circ} 35.5^{\prime}$ & $-159^{\circ} 51.4^{\prime}$ & 1802.6 & 6.3 & 100 & 2.8 & 307 & 8.8 & 108 & $\mathrm{C} / \mathrm{C}$ & \\
\hline 20 & 11 & 1973 & 1110 & $2^{\circ} 35.2^{\prime}$ & $-159^{\circ} 50.0^{\prime}$ & 1804.0 & 6.1 & $\begin{array}{l}109 \\
\end{array}$ & 2.8 & 307 & 8.8 & 115 & $\mathrm{C} / \mathrm{C}$ & \\
\hline 20 & 11 & 1973 & 1130 & $2^{\circ} 34.5^{\prime}$ & $-159^{\circ} 48.0^{\prime}$ & 1806.1 & 6.1 & 117 & 2.8 & 307 & 8.8 & 120 & $\mathrm{C} / \mathrm{C}$ & \\
\hline 20 & 11 & 1973 & 1336 & $2^{\circ} 28.8^{\prime}$ & $-159^{\circ} 36.6^{\prime}$ & 1818.9 & 6.7 & 119 & 2.1 & 304 & 8.8 & 120 & SATL & 1.00 \\
\hline 20 & 11 & 1973 & 1520 & $2^{\circ} 23.2^{\prime}$ & $-159^{\circ} 26.4^{\prime}$ & 1830.5 & 6.5 & 123 & 2.3 & 291 & 8.8 & 120 & SATL & 1.00 \\
\hline 20 & 11 & 1973 & 1732 & $2^{\circ} 15.4^{\prime}$ & $-159^{\circ} 14.4^{\prime}$ & 1844.8 & 6.1 & 124 & 2.7 & 290 & 8.8 & 120 & SATL & 1.00 \\
\hline 20 & 11 & 1973 & 192 & $2^{\circ} 10.2^{\prime}$ & $-159^{\circ} \quad 6.8^{\prime}$ & 1854. & 6.4 & 127 & 2.5 & 282 & 8.8 & 120 & SATL & 1.50 \\
\hline 20 & 11 & 1973 & 206 & $2^{\circ} 6.1^{\prime}$ & $-159^{\circ} 1.3^{\prime}$ & 1860.8 & 6.3 & 106 & 2.5 & 282 & 8.8 & 105 & $\mathrm{C} / \mathrm{C}$ & \\
\hline 20 & 11 & 1973 & 2122 & $2^{\circ} 3.9^{\prime}$ & $-158^{\circ} 53.7^{\prime}$ & 1868.8 & 7.0 & 103 & 1.8 & 292 & 8.8 & 105 & SATL & 1.00 \\
\hline 20 & 11 & 1973 & 2218 & $2^{\circ} \quad 2.4^{\prime}$ & $-158^{\circ} 47.3^{\prime}$ & 1875.3 & 6.8 & 108 & 2.0 & 274 & 8.8 & 105 & SATL & 0.50 \\
\hline 20 & 11 & 1973 & 2323 & $2^{\circ} 0.1^{\prime}$ & $-158^{\circ} 40.3^{\prime}$ & 1882.7 & 7.3 & 143 & 2.0 & 274 & 8.8 & 133 & $\mathrm{C} / \mathrm{C}$ & \\
\hline 21 & 11 & 1973 & 00 & $1^{\circ} 56.5^{\prime}$ & $-158^{\circ} 37.5^{\prime}$ & 1887 & 7.3 & 143 & 2.0 & 274 & 8.8 & 133 & & \\
\hline 21 & 11 & 1973 & 06 & $1^{\circ} 55.9^{\prime}$ & $-158^{\circ} 37.1^{\prime}$ & 1888.0 & 5.9 & 151 & 3.7 & 284 & 8.8 & 133 & SATL & 0.25 \\
\hline 21 & 11 & 1973 & 138 & $1^{\circ} 48.0^{\prime}$ & $-158^{\circ} 32.7^{\prime}$ & 1897. & 5.7 & 146 & 3.7 & 284 & 8.8 & 130 & $\mathrm{C} / \mathrm{C}$ & \\
\hline 21 & 11 & 1973 & 246 & $1^{\circ} 42.6^{\prime}$ & $-158^{\circ} 29.1^{\prime}$ & 1903.5 & 5.0 & 153 & 4.7 & 285 & 8.8 & 130 & SATL & 1.00 \\
\hline 21 & 11 & 1973 & 315 & $1^{\circ} 40.5^{\prime}$ & $-158^{\circ} 28.0^{\prime}$ & 1905. & 4.7 & 145 & 4.7 & 285 & 8.8 & 125 & $\mathrm{C} / \mathrm{C}$ & \\
\hline 21 & 11 & 1973 & & $1^{\circ} 33$ & $-158^{\circ} 22$ & 1914. & 5.1 & 141 & 4.1 & 28 & 8.8 & 125 & SATL & 0.50 \\
\hline 21 & 11 & 1973 & 550 & $1^{\circ} 30.5^{\prime}$ & $-158^{\circ} 20.6^{\prime}$ & 1918. & 4.9 & 132 & 4.1 & 286 & 8.8 & 120 & $\mathrm{C} / \mathrm{C}$ & \\
\hline 21 & 11 & 1973 & 824 & $1^{\circ} 22.1^{\prime}$ & $-158^{\circ} 11.3^{\prime}$ & 1930. & 5.1 & 145 & 4.7 & 273 & 8.8 & 120 & SATL & 1.00 \\
\hline 21 & 11 & 1973 & 954 & $1^{\circ} 15.9^{\prime}$ & $-158^{\circ} \quad 6.9^{\prime}$ & 1938.5 & 5.1 & 145 & 4.7 & 272 & 8.8 & 120 & SATL & 1.50 \\
\hline 21 & 11 & 1973 & 1025 & $1^{\circ} 13.7^{\prime}$ & $-158^{\circ} \quad 5.4^{\prime}$ & 1941.1 & 5.0 & 142 & 4.7 & 272 & 8.8 & 118 & $\mathrm{C} / \mathrm{C}$ & \\
\hline 21 & 11 & 1973 & 1140 & $1^{\circ} 8.8^{\prime}$ & $-158^{\circ} \quad 1.5^{\prime}$ & 1947.4 & 5.0 & 140 & 4.5 & 274 & 8.8 & 118 & SATL & 0.50 \\
\hline 21 & 11 & 1973 & 1410 & $0^{\circ} 59.2^{\prime}$ & $-157^{\circ} 53.4^{\prime}$ & 1960.0 & 5.2 & 137 & 4.2 & 275 & 8.8 & 118 & SATL & 1.50 \\
\hline 21 & 11 & 1973 & 1644 & $0^{\circ} 49.6^{\prime}$ & $-157^{\circ} 44.3^{\prime}$ & 1973.2 & 5.4 & 136 & 4.0 & 273 & 8.8 & 118 & SATL & 1.50 \\
\hline 21 & 11 & 1973 & 1810 & $0^{\circ} 44.0^{\prime}$ & $-157^{\circ} 38.9^{\prime}$ & 1981.0 & 5.2 & 136 & 4.2 & 275 & 8.8 & 118 & SATL & 0.50 \\
\hline 21 & 11 & 1973 & 1848 & $0^{\circ} 41.6^{\prime}$ & $-157^{\circ} 36.6^{\prime}$ & 1984.3 & 5.4 & 136 & 4.0 & 273 & 8.8 & 118 & SATL & 0.25 \\
\hline 21 & 11 & 1973 & 2034 & $0^{\circ} 34.7^{\prime}$ & $-157^{\circ} 29.9^{\prime}$ & 1993.9 & 5.8 & 139 & 3.9 & 267 & 8.8 & 118 & SATL & 1.00 \\
\hline 21 & 11 & 1973 & 220 & $0^{\circ} 28.5^{\prime}$ & $-157^{\circ} 24.4^{\prime}$ & 2002.2 & 5.9 & 141 & 3.9 & 267 & 8.8 & 120 & $\mathrm{C} / \mathrm{C}$ & \\
\hline
\end{tabular}


TABLE 1 - Continued

\begin{tabular}{|c|c|c|c|c|c|c|c|c|c|c|c|c|c|c|}
\hline & & & & & & & & ual & Dri & & & & & \\
\hline DA & MO & YR & Time & Latitude & Longitude & Dist. & Speed & Course & Speed & Hed. & Speed & Course & Comment & Qlty \\
\hline 21 & 11 & 1973 & 2320 & $0^{\circ} 22.3^{\prime}$ & $-157^{\circ} 19.5^{\prime}$ & 2010.1 & 6.1 & 140 & 3.8 & 266 & 8.8 & 120 & SATL & 0.25 \\
\hline 22 & 11 & 1973 & 00 & $0^{\circ} 19.2^{\prime}$ & $-157^{\circ} 16.9^{\prime}$ & 2014.1 & 6.1 & 140 & 3.8 & 266 & 8.8 & 120 & & \\
\hline 22 & 11 & 1973 & 138 & $0^{\circ} 11.6^{\prime}$ & $-157^{\circ} 10.6^{\prime}$ & 2024.0 & 6.3 & 138 & 3.4 & 264 & 8.8 & 120 & SATL & 0.25 \\
\hline 22 & 11 & 1973 & 420 & $0^{\circ}-1.2^{\prime}$ & $-156^{\circ} 59.2^{\prime}$ & 2041.1 & 6.6 & 136 & 3.0 & 264 & 8.8 & 120 & SATL & 0.25 \\
\hline 22 & 11 & 1973 & 443 & $0^{\circ}-3.0^{\prime}$ & $-156^{\circ} 57.4^{\prime}$ & 2043.7 & 8.2 & 355 & 3.0 & 264 & 8.8 & 15 & $\mathrm{C} / \mathrm{C}$ & \\
\hline 22 & 11 & 1973 & 557 & $0^{\circ}-7.1^{\prime}$ & $-156^{\circ} 58.3^{\prime}$ & 2053.8 & 11.8 & 268 & 3.0 & 264 & 8.8 & 270 & $\mathrm{C} / \mathrm{C}$ & $\cdot$ \\
\hline 22 & 11 & 1973 & 66 & $0^{\circ} \quad 7.0^{\prime}$ & $\begin{array}{ll}-157^{\circ} & 0.1^{\prime}\end{array}$ & 2055.6 & 10.6 & 266 & 1.9 & 248 & 8.8 & 270 & SATL & 0.50 \\
\hline 22 & 11 & 1973 & 658 & $0^{\circ} 6.4^{\prime}$ & $-157^{\circ} \quad 9.3^{\prime}$ & 2064.8 & 6.9 & 123 & 1.9 & 248 & 8.2 & 112 & $\mathrm{C} / \mathrm{CS}$ & \\
\hline 22 & 11 & 1973 & 72 & $0^{\circ} 6.1^{\prime}$ & $-157^{\circ} 8.9^{\prime}$ & 2065.2 & 3.9 & 132 & 1.9 & 248 & 5.0 & 112 & $\mathrm{C} / \mathrm{S}$ & \\
\hline 22 & 11 & 1973 & 78 & $0^{\circ} \quad 5.9^{\prime}$ & $-157^{\circ} 8.6^{\prime}$ & 2065.6 & 3.5 & 117 & 1.9 & 248 & 5.0 & 100 & $\mathrm{C} / \mathrm{C}$ & \\
\hline 22 & 11 & 1973 & 723 & $0^{\circ} 5.5^{\prime}$ & $-157^{\circ} \quad 7.8^{\prime}$ & 2066.5 & 3.9 & 115 & 1.9 & 248 & 5.4 & 100 & $\mathrm{C} / \mathrm{S}$ & \\
\hline 22 & 11 & 1973 & 725 & $0^{\circ} 5.4^{\prime}$ & $-157^{\circ} \quad 7.7^{\prime}$ & 2066.6 & 5.4 & 100 & 0.0 & 0 & 5.4 & 100 & S316 & 1.50 \\
\hline 22 & 11 & 1973 & 725 & $0^{\circ} \quad 5.4^{\prime}$ & $-157^{\circ} 7.7^{\prime}$ & 2066.6 & 0.0 & 0 & 0.0 & 0 & 0.0 & 500 & STOP & \\
\hline 27 & 11 & 1973 & 45 & $0^{\circ} 5.4^{\prime}$ & $-157^{\circ} 7.7^{\prime}$ & 2066.6 & 1.7 & 273 & 1.7 & 273 & 0.0 & 500 & DEP & 1.50 \\
\hline 27 & 11 & 1973 & 45 & $0^{\circ} 5.4^{\prime}$ & $\begin{array}{lll}-157^{\circ} & 7.7^{\prime}\end{array}$ & 2066.6 & 9.3 & 195 & 1.7 & 273 & 9.1 & 185 & $\mathrm{U} / \mathrm{W}$ & \\
\hline 27 & 11 & 1973 & 540 & $0^{\circ}-8.8^{\prime}$ & $-157^{\circ} 11.6^{\prime}$ & 2081.3 & 9.6 & 195 & 1.7 & 263 & 9.1 & 185 & SATL & 0.25 \\
\hline 27 & 11 & 1973 & 60 & $0^{\circ}-11.9^{\prime}$ & $-157^{\circ} 12.4^{\prime}$ & 2084.5 & 9.8 & 203 & 1.7 & 263 & 9.1 & 194 & $\mathrm{C} / \mathrm{C}$ & \\
\hline 27 & 11 & 1973 & 626 & $0^{\circ}-15.8^{\prime}$ & $-157^{\circ} 14.1^{\prime}$ & 2088.8 & 9.1 & 196 & 0.2 & 283 & 9.1 & 194 & SATL & 1.50 \\
\hline 27 & 11 & 1973 & 720 & $0^{\circ}-23.7^{\prime}$ & $-157^{\circ} 16.3^{\prime}$ & 2097.0 & 9.1 & 199 & 0.9 & 288 & 9.1 & 194 & SATL & 1.00 \\
\hline 27 & 11 & 1973 & 753 & $0^{\circ}-28.4^{\prime}$ & $-157^{\circ} 18.0^{\prime}$ & 2102.0 & 9.2 & 209 & 0.9 & 288 & 9.1 & 204 & $\mathrm{C} / \mathrm{C}$ & \\
\hline 27 & 11 & 1973 & 816 & $0^{\circ}-31.5^{\prime}$ & $-157^{\circ} 19.7^{\prime}$ & 2105.5 & 10.2 & 211 & 1.6 & 252 & 9.1 & 204 & SATL & 0.25 \\
\hline 27 & 11 & 1973 & 1024 & $0^{\circ}-50.3^{\prime}$ & $-157^{\circ} 30.8^{\prime}$ & 2127.4 & 9.9 & 209 & 1.2 & 255 & 9.1 & 204 & SATL & 1.50 \\
\hline 27 & 11 & 1973 & 1130 & $0^{\circ}-59.8^{\prime}$ & $-157^{\circ} 36.1^{\prime}$ & 2138.2 & 9.8 & 203 & 1.2 & 255 & 9.1 & 197 & $\mathrm{C} / \mathrm{C}$ & \\
\hline 27 & 11 & 1973 & 1252 & $-1^{\circ} 12.1^{\prime}$ & $-157^{\circ} 41.3^{\prime}$ & 2151.6 & 9.9 & 204 & 1.4 & 256 & 9.1 & 197 & SATL & 0.50 \\
\hline 27 & 11 & 1973 & 1434 & $-1^{\circ} 27.5^{\prime}$ & $-157^{\circ} 48.2^{\prime}$ & 2168.5 & 10.3 & 203 & 1.6 & 241 & 9.1 & 197 & SATL & 0.50 \\
\hline 27 & 11 & 1973 & 1526 & $-1^{\circ} 35.7^{\prime}$ & $-157^{\circ} 51.7^{\prime}$ & 2177.4 & 10.4 & 204 & 1.8 & 244 & 9.1 & 197 & SATL & 0.50 \\
\hline 27 & 11 & 1973 & 1712 & $-1^{\circ} 52.5^{\prime}$ & $-157^{\circ} 59.3^{\prime}$ & 2195.8 & 10.5 & 203 & 1.7 & 238 & 9.1 & 197 & SATL & 1.00 \\
\hline 27 & 11 & 1973 & 180 & $\begin{array}{lll}-2^{\circ} & 0.2^{\prime}\end{array}$ & $\begin{array}{ll}-158^{\circ} \quad 2.6^{\prime} \\
\end{array}$ & 2204.2 & 10.5 & 202 & 1.7 & 234 & 9.1 & 197 & SATL & 0.50 \\
\hline 27 & 11 & 1973 & 1854 & $-2^{\circ} 8.9^{\prime}$ & $-158^{\circ} \quad 6.2^{\prime}$ & 2213.6 & 10.4 & 202 & 1.6 & 233 & 9.1 & 197 & SATL & 1.00 \\
\hline 27 & 11 & 1973 & 2042 & $-2^{\circ} 26.3^{\prime}$ & $-158^{\circ} 13.3^{\prime}$ & 2232.4 & 10.7 & 201 & 1.7 & 221 & 9.1 & 197 & SATL & 1.00 \\
\hline 27 & 11 & 1973 & 2132 & $-2^{\circ} 34.6^{\prime}$ & $-158^{\circ} 16.4^{\prime}$ & 2241.3 & 10.7 & 202 & 1.7 & 221 & 9.1 & 199 & $\mathrm{C} / \mathrm{C}$ & \\
\hline 27 & 11 & 1973 & 2158 & $-2^{\circ} 38.9^{\prime}$ & $-158^{\circ} 18.2^{\prime}$ & 2245.9 & 10.6 & 203 & 1.6 & 224 & 9.1 & 199 & SATL & 0.50 \\
\hline 27 & 11 & 1973 & 2348 & $-2^{\circ} 56.8^{\prime}$ & $-158^{\circ} 25.7^{\prime}$ & 2265.3 & 10.5 & 201 & 1.4 & 214 & 9.1 & 199 & SATL & 0.50 \\
\hline 28 & 11 & 1973 & 00 & $-2^{\circ} 58.8^{\prime}$ & $-158^{\circ} 26.4^{\prime}$ & 2267.4 & 10.5 & 201 & 1.4 & 214 & 9.1 & 199 & & \\
\hline 28 & 11 & 1973 & 010 & $\begin{array}{ll}-3^{\circ} & 0.4^{\prime}\end{array}$ & $-158^{\circ} 27.1^{\prime}$ & 2269.2 & 10.5 & 205 & 1.4 & 214 & 9.1 & 204 & $\mathrm{C} / \mathrm{C}$ & \\
\hline 28 & 11 & 1973 & 24 & $-3^{\circ} 18.4^{\prime}$ & $-158^{\circ} 35.6^{\prime}$ & 2289.1 & 10.3 & 208 & 1.3 & 235 & 9.1 & 204 & SATL & 0.50 \\
\hline 28 & 11 & 1973 & 446 & $-3^{\circ} 42.9^{\prime}$ & $-158^{\circ} 48.6^{\prime}$ & 2316.8 & 9.3 & 203 & 0.3 & 174 & 9.1 & 204 & SATL & 1.00 \\
\hline 28 & 11 & 1973 & 530 & $-3^{\circ} 49.2^{\prime}$ & $-158^{\circ} 51.3^{\prime}$ & 2323.7 & 9.7 & 210 & 1.1 & 263 & 9.1 & 204 & SATL & 1.00 \\
\hline 28 & 11 & 1973 & 530 & $-3^{\circ} 49.2^{\prime}$ & $-158^{\circ} 51.3^{\prime}$ & 2323.7 & 9.7 & 206 & 1.1 & 263 & 9.1 & 200 & $\mathrm{C} / \mathrm{C}$ & \\
\hline 28 & 11 & 1973 & 628 & $-3^{\circ} 57.6^{\prime}$ & $-158^{\circ} 55.4^{\prime}$ & 2333.0 & 9.3 & 202 & 0.4 & 254 & 9.1 & 200 & SATL & 1.50 \\
\hline 28 & 11 & 1973 & 720 & $-4^{\circ} 5.1^{\prime}$ & $-158^{\circ} 58.4^{\prime}$ & 2341.0 & 9.8 & 206 & 1.2 & 259 & 9.1 & 200 & SATL & 1.00 \\
\hline 28 & 11 & 1973 & 818 & $-4^{\circ} 13.6^{\prime}$ & $\begin{array}{ll}-159^{\circ} \quad 2.6^{\prime} \\
\end{array}$ & 2350.6 & 9.3 & 202 & 0.4 & 267 & 9.1 & 200 & SATL & 1.00 \\
\hline 28 & 11 & 1973 & 818 & $-4^{\circ} 13.6^{\prime}$ & 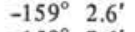 & 2350.6 & 9.3 & 204 & 0.4 & 267 & 9.1 & 202 & $\mathrm{C} / \mathrm{C}$ & \\
\hline 28 & 11 & 1973 & 936 & $-4^{\circ} 24.6^{\prime}$ & $-159^{\circ} 7.6^{\prime}$ & 2362.6 & 9.3 & 206 & 0.7 & 279 & 9.1 & 202 & SATL & 1.50 \\
\hline 28 & 11 & 1973 & 1122 & $-4^{\circ} 39.3^{\prime}$ & $-159^{\circ} 14.9^{\prime}$ & 2379.0 & 9.1 & 203 & 0.1 & 310 & 9.1 & 202 & SATL & 1.00 \\
\hline 28 & 11 & 1973 & 1322 & $-4^{\circ} 56.1^{\prime}$ & $-159^{\circ} 21.9^{\prime}$ & 2397.2 & 9.1 & 201 & 0.1 & 310 & 9.1 & 200 & $\mathrm{C} / \mathrm{C}$ & \\
\hline 28 & 11 & 1973 & 1328 & $-4^{\circ} 56.9^{\prime}$ & $-159^{\circ} 22.2^{\prime}$ & 2398.1 & 9.0 & 203 & 0.5 & 298 & 9.1 & 200 & SATL & 0.50 \\
\hline 28 & 11 & 1973 & 140 & $\begin{array}{ll}-5^{\circ} & 1.3^{\prime}\end{array}$ & $-159^{\circ} 24.1^{\prime}$ & 2402.9 & 9.1 & 205 & 0.5 & 298 & 9.1 & 202 & $\mathrm{C} / \mathrm{C}$ & \\
\hline 28 & 11 & 1973 & 1512 & $-5^{\circ} 11.2^{\prime}$ & $-159^{\circ} 28.7^{\prime}$ & 2413.8 & 9.9 & 205 & 0.9 & 232 & 9.1 & 202 & SATL & 0.00 \\
\hline 28 & 11 & 1973 & 1620 & $-5^{\circ} 21.4^{\prime}$ & $-159^{\circ} 33.4^{\prime}$ & 2425.0 & 8.6 & 203 & 0.6 & 5 & 9.1 & 202 & SATL & 1.00 \\
\hline 28 & 11 & 1973 & 178 & $-5^{\circ} 27.7^{\prime}$ & $-159^{\circ} 36.1^{\prime}$ & 2431.9 & 9.3 & 206 & 0.6 & 276 & 9.1 & 202 & SATL & 0.50 \\
\hline 28 & 11 & 1973 & 180 & $-5^{\circ} 35.0^{\prime}$ & $-159^{\circ} 39.6^{\prime}$ & 2439.9 & 9.3 & 208 & 0.6 & 276 & 9.1 & 204 & $\mathrm{C} / \mathrm{C}$ & \\
\hline 28 & 11 & 1973 & 1812 & $-5^{\circ} 36.6^{\prime}$ & $-159^{\circ} 40.5^{\prime}$ & 2441.8 & 9.1 & 206 & 0.3 & 302 & 9.1 & 204 & SATL & 1.00 \\
\hline 28 & 11 & 1973 & 1854 & $-5^{\circ} 42.3^{\prime}$ & $-159^{\circ} 43.3^{\prime}$ & 2448.1 & 9.0 & 206 & 0.3 & 305 & 9.1 & 204 & SATL & 0.50 \\
\hline 28 & 11 & 1973 & 1950 & $-5^{\circ} 49.9^{\prime}$ & $-159^{\circ} 47.0^{\prime}$ & 2456.6 & 9.1 & 206 & 0.3 & 289 & 9.1 & 204 & SATL & 0.25 \\
\hline 28 & 11 & 1973 & 2114 & $\begin{array}{lll}-6^{\circ} & 1.4^{\prime}\end{array}$ & $-159^{\circ} 52.6^{\prime}$ & 2469.3 & 8.8 & 207 & 0.6 & 322 & 9.1 & 204 & SATL & 0.25 \\
\hline 28 & 11 & 1973 & 230 & $-6^{\circ} 15.3^{\prime}$ & $-159^{\circ} 59.8^{\prime}$ & 2485.0 & 8.9 & 208 & 0.7 & 308 & 9.1 & 204 & SATL & 0.25 \\
\hline 29 & 11 & 1973 & 00 & $-6^{\circ} 23.2^{\prime}$ & $-160^{\circ} 4.1^{\prime}$ & 2493.9 & 8.9 & 208 & 0.7 & 308 & 9.1 & 204 & & \\
\hline 29 & 11 & 1973 & 056 & $-6^{\circ} 30.5^{\prime}$ & $-160^{\circ} 8.1^{\prime}$ & 2502.3 & 9.0 & 209 & 0.8 & 303 & 9.1 & 204 & SATL & 0.50 \\
\hline 29 & 11 & 1973 & 136 & $-6^{\circ} 35.7^{\prime}$ & $-160^{\circ} 11.0^{\prime}$ & 2508.3 & 9.0 & 206 & 0.8 & 303 & 9.1 & 201 & $\mathrm{C} / \mathrm{C}$ & \\
\hline 29 & 11 & 1973 & 242 & $-6^{\circ} 44.6^{\prime}$ & $-160^{\circ} 15.4^{\prime}$ & 2518.1 & 8.8 & 206 & 0.9 & 315 & 9.1 & 201 & SATL & 0.50 \\
\hline 29 & 11 & 1973 & 310 & $-6^{\circ} 48.3^{\prime}$ & $-160^{\circ} 17.2^{\prime}$ & 2522.2 & 8.7 & 202 & 0.9 & 315 & 9.1 & 197 & $\mathrm{C} / \mathrm{C}$ & \\
\hline 29 & 11 & 1973 & 358 & $-6^{\circ} 54.7^{\prime}$ & $-160^{\circ} 19.9^{\prime}$ & 2529.2 & 8.7 & 203 & 1.0 & 316 & 9.1 & 197 & SATL & 1.00 \\
\hline 29 & 11 & 1973 & 622 & $-7^{\circ} 13.9^{\prime}$ & $-160^{\circ} 28.0^{\prime}$ & 2550.0 & 8.9 & 204 & 1.1 & 304 & 9.1 & 197 & SATL & 0.25 \\
\hline 29 & 11 & 1973 & 722 & $-7^{\circ} 22.0^{\prime}$ & $-160^{\circ} 31.6^{\prime}$ & 2558.8 & 8.2 & 200 & 1.0 & 353 & 9.1 & 197 & SATL & 1.50 \\
\hline 29 & 11 & 1973 & 749 & $-7^{\circ} 25.5^{\prime}$ & $-160^{\circ} 32.9^{\prime}$ & 2562.8 & 8.3 & 203 & 1.0 & 353 & 9.1 & 200 & $\mathrm{C} / \mathrm{C}$ & \\
\hline 29 & 11 & 1973 & 812 & $-7^{\circ} 28.4^{\prime}$ & $-160^{\circ} 34.1^{\prime}$ & 2565.7 & 9.4 & 205 & 0.9 & 270 & 9.1 & 200 & SATL & 0.25 \\
\hline 29 & 11 & 1973 & 852 & $-7^{\circ} 34.1^{\prime}$ & $-160^{\circ} 36.8^{\prime}$ & 2572.0 & 8.8 & 204 & 0.7 & 320 & 9.1 & 200 & SATL & 0.25 \\
\hline 29 & 11 & 1973 & 1012 & $-7^{\circ} 44.7^{\prime}$ & $-160^{\circ} 41.6^{\prime}$ & 2583.7 & 8.7 & 203 & 0.7 & 320 & 9.1 & 199 & $\mathrm{C} / \mathrm{C}$ & \\
\hline 29 & 11 & 1973 & 1034 & $-7^{\circ} 47.7^{\prime}$ & $-160^{\circ} 42.9^{\prime}$ & 2586.9 & 9.0 & 202 & 0.6 & 303 & 9.1 & 199 & SATL & 1.50 \\
\hline 29 & 11 & 1973 & 142 & $-8^{\circ} 16.5^{\prime}$ & $-160^{\circ} 54.9^{\prime}$ & 2618.0 & 9.3 & 201 & 0.5 & 261 & 9.1 & 199 & SATL & 1.00 \\
\hline 29 & 11 & 1973 & 150 & $-8^{\circ} 24.9^{\prime}$ & $-160^{\circ} 58.2^{\prime}$ & 2627.1 & 9.4 & 206 & 0.5 & 261 & 9.1 & 204 & $\mathrm{C} / \mathrm{C}$ & \\
\hline 29 & 11 & 1973 & 1538 & $-8^{\circ} 30.2^{\prime}$ & $\begin{array}{ll}-161^{\circ} & 0.9^{\prime}\end{array}$ & 2633.0 & 9.1 & 205 & 0.2 & 283 & 9.1 & 204 & SATL & 0.50 \\
\hline 29 & 11 & 1973 & 1724 & $-8^{\circ} 44.8^{\prime}$ & $-161^{\circ} 7.9^{\prime}$ & 2649.1 & 9.5 & 207 & 0.6 & 252 & 9.1 & 204 & SATL & 1.50 \\
\hline 29 & 11 & 1973 & 190 & $-8^{\circ} 58.4^{\prime}$ & $-161^{\circ} 14.8^{\prime}$ & 2664.4 & 9.2 & 206 & 0.3 & 272 & 9.1 & 204 & SATL & 1.00 \\
\hline 29 & 11 & 1973 & 2052 & $-9^{\circ} 13.9^{\prime}$ & $-161^{\circ} 22.3^{\prime}$ & 2681.5 & 9.4 & 206 & 0.5 & 256 & 9.1 & 204 & SATL & 0.25 \\
\hline 29 & 11 & 1973 & 226 & $-9^{\circ} 24.3^{\prime}$ & $-161^{\circ} 27.5^{\prime}$ & 2693.1 & 9.5 & 205 & 0.4 & 228 & 9.1 & 204 & SATL & 1.50 \\
\hline 29 & 11 & 1973 & 2352 & $-9^{\circ} 39.5^{\prime}$ & $-161^{\circ} 34.7^{\prime}$ & 2709.9 & 9.3 & 205 & 0.3 & 252 & 9.1 & 204 & SATL & 0.50 \\
\hline 30 & 11 & 1973 & 00 & $-9^{\circ} 40.6^{\prime}$ & $-161^{\circ} 35.2^{\prime}$ & 2711.2 & 9.3 & 205 & 0.3 & 252 & 9.1 & 204 & & \\
\hline 30 & 11 & 1973 & 134 & $-9^{\circ} 53.8^{\prime}$ & $-161^{\circ} 41.6^{\prime}$ & 2725.7 & 9.1 & 206 & 0.3 & 292 & 9.1 & 204 & SATL & 1.00 \\
\hline 30 & 11 & 1973 & 310 & $-10^{\circ} \quad 6.9^{\prime}$ & $-161^{\circ} 48.1^{\prime}$ & 2740.3 & 9.6 & 208 & 0.8 & 255 & 9.1 & 204 & SATL & 0.00 \\
\hline 30 & 11 & 1973 & 452 & $-10^{\circ} 21.4^{\prime}$ & $-161^{\circ} 55.9^{\prime}$ & 2756.7 & 8.7 & 195 & 1.5 & 95 & 9.1 & 204 & SATL & 1.50 \\
\hline
\end{tabular}


TABLE 1 - Continued

\begin{tabular}{|c|c|c|c|c|c|c|c|c|c|c|c|c|c|c|c|}
\hline & & & & & & & & & Dri & & & & & & Dri \\
\hline DA & MO & YR & Time & Latitude & Longitude & Dist. & Speed & Course & Speed & Hed. & Speed & Course & Comment & Qlty. & Dist. \\
\hline 30 & 11 & 1973 & 524 & $-10^{\circ} 25.9^{\prime}$ & $-161^{\circ} 57.1^{\prime}$ & 2761.4 & 9.3 & 202 & 0.4 & 144 & 9.1 & 204 & SATL & 1.00 & 0.8 \\
\hline 30 & 11 & 1973 & 546 & $-10^{\circ} 29.1^{\prime}$ & $-161^{\circ} 58.4^{\prime}$ & 2764.8 & 9.1 & 233 & 0.4 & 144 & 9.1 & 235 & $\mathrm{C} / \mathrm{C}$ & & \\
\hline 30 & 11 & 1973 & 630 & $-10^{\circ} 33.1^{\prime}$ & $-162^{\circ} 3.8^{\prime}$ & 2771.5 & 9.1 & 231 & 0.6 & 143 & 9.1 & 235 & SATL & 1.00 & 0.4 \\
\hline 30 & 11 & 1973 & 710 & $-10^{\circ} 36.9^{\prime}$ & $-162^{\circ} 8.6^{\prime}$ & 2777.5 & 9.3 & 232 & 0.5 & 170 & 9.1 & 235 & SATL & 1.50 & 0.5 \\
\hline 30 & 11 & 1973 & 745 & $-10^{\circ} 40.2^{\prime}$ & $-162^{\circ} 13.0^{\prime}$ & 2783.0 & 9.3 & 240 & 0.5 & 170 & 9.1 & 243 & $\mathrm{C} / \mathrm{C}$ & & \\
\hline 30 & 11 & 1973 & 816 & $-10^{\circ} 42.6^{\prime}$ & $-162^{\circ} 17.2^{\prime}$ & 2787.7 & 9.5 & 244 & 0.5 & 268 & 9.1 & 243 & SATL & 1.00 & 0.6 \\
\hline 30 & 11 & 1973 & 914 & $-10^{\circ} 46.6^{\prime}$ & $-162^{\circ} 25.6^{\prime}$ & 2796.9 & 8.8 & 145 & 0.5 & 268 & 9.1 & 143 & $\mathrm{C} / \mathrm{C}$ & & \\
\hline 30 & 11 & 1973 & 946 & $-10^{\circ} 50.5^{\prime}$ & $-162^{\circ} 22.9^{\prime}$ & 2801.7 & 8.7 & 144 & 0.4 & 301 & 9.1 & 143 & SATL & 0.50 & 0.7 \\
\hline 30 & 11 & 1973 & 1134 & $-11^{\circ} 3.2^{\prime}$ & $-162^{\circ} 13.5^{\prime}$ & 2817.4 & 10.7 & 141 & 1.4 & 130 & 9.1 & 143 & SATL & 1.00 & 0.8 . \\
\hline 30 & 11 & 1973 & 1140 & $-11^{\circ} 4.0^{\prime}$ & $-162^{\circ} 12.8^{\prime}$ & 2828.4 & 7.7 & 310 & 1.4 & 130 & 9.1 & 310 & $\mathrm{C} / \mathrm{C}$ & & \\
\hline 30 & 11 & 1973 & 1150 & $-11^{\circ} 3.2^{\prime}$ & $-162^{\circ} 13.8^{\prime}$ & 2819.7 & 5.6 & 310 & 1.4 & 130 & 7.0 & 310 & $\mathrm{C} / \mathrm{S}$ & & \\
\hline 30 & 11 & 1973 & 122 & $-11^{\circ} 2.5^{\prime}$ & $-162^{\circ} 14.7^{\prime}$ & 2820.8 & 5.8 & 341 & 1.4 & 130 & 7.0 & 335 & $\mathrm{C} / \mathrm{C}$ & & \\
\hline 30 & 11 & 1973 & 127 & $-11^{\circ} 2.0^{\prime}$ & $-162^{\circ} 14.9^{\prime}$ & 2821.3 & 5.7 & 335 & 1.4 & 130 & 7.0 & 330 & $\mathrm{C} / \mathrm{C}$ & & \\
\hline 30 & 11 & 1973 & 1229 & $-11^{\circ} 0.1^{\prime}$ & $-162^{\circ} 15.8^{\prime}$ & 2823.4 & 7.0 & 330 & 0.0 & 0 & 7.0 & 330 & S317 & 1.50 & 1.3 \\
\hline 30 & 11 & 1973 & 1229 & $-11^{\circ} 0.1^{\prime}$ & $-162^{\circ} 15.8^{\prime}$ & 2823.4 & 0.0 & 0 & 0.0 & 0 & 0.0 & 500 & STOP & & \\
\hline 8 & 12 & 1973 & 428 & $-11^{\circ} 0.1^{\prime}$ & $-162^{\circ} 15.8^{\prime}$ & 2823.4 & 0.3 & 231 & 0.3 & 231 & 0.0 & 500 & DEP & 1.50 & 0.1 \\
\hline 8 & 12 & 1973 & 428 & $-11^{\circ} 0.1^{\prime}$ & $-162^{\circ} 15.8^{\prime}$ & 2823.4 & 7.7 & 106 & 0.3 & 231 & 7.9 & 104 & $U / W$ & & \\
\hline 8 & 12 & 1973 & 446 & $-11^{\circ} 0.7^{\prime}$ & $-162^{\circ} 13.5^{\prime}$ & 2825.7 & 9.2 & 102 & 0.3 & 231 & 9.4 & 100 & $\mathrm{C} / \mathrm{CS}$ & & \\
\hline 8 & 12 & 1973 & 642 & $-11^{\circ} 4.3^{\prime}$ & $-161^{\circ} 55.8^{\prime}$ & 2843.5 & 9.0 & 102 & 0.5 & 244 & 9.4 & 100 & SATL & 1.50 & 0.8 \\
\hline 8 & 12 & 1973 & 846 & $-11^{\circ} 8.1^{\prime}$ & $-161^{\circ} 37.2^{\prime}$ & 2862.1 & 9.2 & 99 & 0.3 & 331 & 9.4 & 100 & SATL & 0.00 & 1.0 \\
\hline 8 & 12 & 1973 & 936 & $-11^{\circ} 9.3^{\prime}$ & $-161^{\circ} 29.4^{\prime}$ & 2869.8 & 9.2 & 102 & 0.3 & 331 & 9.4 & 103 & $\mathrm{C} / \mathrm{C}$ & & \\
\hline 8 & 12 & 197.3 & 1026 & $-11^{\circ} 10.8^{\prime}$ & $-161^{\circ} 21.8^{\prime}$ & 2877.5 & 8.8 & 105 & 0.7 & 257 & 9.4 & 103 & SATL & 1.50 & 0.5 \\
\hline 8 & 12 & 1973 & 110 & $-11^{\circ} 12.1^{\prime}$ & $-161^{\circ} 16.9^{\prime}$ & 2882.5 & 8.8 & 106 & 0.7 & 257 & 9.4 & 104 & $\mathrm{C} / \mathrm{C}$ & & \\
\hline 8 & 12 & 1973 & 1232 & $-11^{\circ} 15.8^{\prime}$ & $-161^{\circ} 3.6^{\prime}$ & 2896.0 & 8.8 & 105 & 0.6 & 267 & 9.4 & 104 & SATL & 1.50 & 1.4 \\
\hline 8 & 12 & 1973 & 136 & $-11^{\circ} 17.1^{\prime}$ & $-160^{\circ} 58.7^{\prime}$ & 2901.0 & $\begin{array}{l}.00 \\
8.8\end{array}$ & 104 & 0.6 & 267 & 9.4 & 103 & $\mathrm{C} / \mathrm{C}$ & & \\
\hline 8 & 12 & 1973 & 1526 & $-11^{\circ} 22.1^{\prime}$ & $-160^{\circ} 38.4^{\prime}$ & 2921.5 & 9.2 & 105 & 0.3 & 231 & 9.4 & 103 & SATL & 1.50 & 1.9 \\
\hline 8 & 12 & 1973 & 160 & $-11^{\circ} 23.4^{\prime}$ & $-160^{\circ} 33.2^{\prime}$ & 2926.8 & 9.2 & 102 & 0.3 & 231 & 9.4 & 101 & $\mathrm{C} / \mathrm{C}$ & & \\
\hline 8 & 12 & 1973 & 1648 & $-11^{\circ} 25.0^{\prime}$ & $-160^{\circ} 25.9^{\prime}$ & 2934.1 & 8.9 & 105 & 0.8 & 236 & 9.4 & 101 & SATL & 1.50 & 0.5 \\
\hline 8 & 12 & 1973 & 1712 & $-11^{\circ} 25.9^{\prime}$ & $-160^{\circ} 22.4^{\prime}$ & 2937.7 & 9.1 & 104 & 0.5 & 227 & 9.4 & 101 & SATL & 1.50 & 0.4 \\
\hline 8 & 12 & 1973 & 1824 & $-11^{\circ} 28.5^{\prime}$ & $-160^{\circ} 11.6^{\prime}$ & 2948.6 & 9.1 & 103 & 0.4 & 226 & 9.4 & 101 & SATL & 1.00 & 0.7 \\
\hline 8 & 12 & 1973 & 2158 & $-11^{\circ} 36.0^{\prime}$ & $-159^{\circ} 39.2^{\prime}$ & 2981.2 & 8.9 & 104 & 0.7 & 242 & 9.4 & 101 & SATL & 0.25 & 1.7 \\
\hline 9 & 12 & 1973 & 00 & $-11^{\circ} 40.3^{\prime}$ & $-159^{\circ} 21.3^{\prime}$ & 2999.2 & 8.9 & 104 & 0.7 & 242 & 9.4 & 101 & & & \\
\hline 9 & 12 & 1973 & 06 & $-11^{\circ} 40.5^{\prime}$ & $-159^{\circ} 20.4^{\prime}$ & 3000.1 & 7.9 & 105 & 1.6 & 259 & 9.4 & 101 & SATL & 0.50 & 1.5 \\
\hline 9 & 12 & 1973 & 442 & $-11^{\circ} 50.2^{\prime}$ & $-158^{\circ} 44.6^{\prime}$ & 3036.5 & 8.2 & 107 & 1.6 & 247 & 9.4 & 101 & SATL & 0.25 & 7.6 \\
\hline 9 & 12 & 1973 & 530 & $-11^{\circ} 52.1^{\prime}$ & $-158^{\circ} 38.2^{\prime}$ & 3043.0 & 8.1 & 105 & 1.6 & 247 & 9.4 & 99 & $\mathrm{C} / \mathrm{C}$ & & \\
\hline 9 & 12 & 1973 & 552 & $-11^{\circ} 52.9^{\prime}$ & $-158^{\circ} 35.3^{\prime}$ & 3046.0 & 7.8 & 102 & 1.6 & 263 & 9.4 & 99 & SATL & 1.50 & 1.9 \\
\hline 9 & 12 & 1973 & 740 & $-11^{\circ} 55.9^{\prime}$ & $-158^{\circ} 21.2^{\prime}$ & 3060.1 & 8.1 & 103 & 1.5 & 257 & 9.4 & 99 & SATL & 1.00 & 3.0 \\
\hline 9 & 12 & 1973 & 835 & $-11^{\circ} 57.5^{\prime}$ & $-158^{\circ} 13.8^{\prime}$ & 3067.5 & 8.0 & 99 & 1.5 & 257 & 9.4 & 96 & $\mathrm{C} / \mathrm{C}$ & & \\
\hline 9 & 12 & 1973 & 1140 & $-12^{\circ} \quad 1.6^{\prime}$ & $-157^{\circ} 48.9^{\prime}$ & 3092.2 & 8.0 & 99 & 1.5 & 258 & 9.4 & 96 & SATL & 1.50 & 5.9 \\
\hline 9 & 12 & 1973 & 1326 & $-12^{\circ} 3.9^{\prime}$ & $-157^{\circ} 34.7^{\prime}$ & 3106.3 & 8.5 & 98 & 1.0 & 255 & 9.4 & 96 & SATL & 1.50 & 2.7 \\
\hline 9 & 12 & 1973 & 1438 & $-12^{\circ} 5.4^{\prime}$ & $-157^{\circ} 24.4^{\prime}$ & 3116.5 & 8.5 & 97 & 0.9 & 267 & 9.4 & 96 & SATL & 1.00 & 1.3 \\
\hline 9 & 12 & 1973 & 1615 & $-12^{\circ} 7.1^{\prime}$ & $-157^{\circ} 10.4^{\prime}$ & 3130.2 & 8.5 & 101 & 0.9 & 267 & 9.4 & 100 & $\mathrm{C} / \mathrm{C}$ & & \\
\hline 9 & 12 & 1973 & 1734 & $-12^{\circ} 9.3^{\prime}$ & $-156^{\circ} 59.2^{\prime}$ & 3141.4 & 8.2 & 100 & 1.2 & 277 & 9.4 & 100 & SATL & 1.00 & 2.7 \\
\hline 9 & 12 & 1973 & 1920 & $-12^{\circ} 11.9^{\prime}$ & $-156^{\circ} 44.7^{\prime}$ & 3155.9 & 8.1 & 103 & 1.4 & 263 & 9.4 & 100 & SATL & 1.00 & 2.2 \\
\hline 9 & 12 & 1973 & 1956 & $-12^{\circ} 13.0^{\prime}$ & $-156^{\circ} 39.9^{\prime}$ & 3160.7 & 8.1 & 109 & 1.4 & 263 & 9.4 & 105 & $\mathrm{C} / \mathrm{C}$ & & \\
\hline 9 & 12 & 1973 & 2230 & $-12^{\circ} 19.6^{\prime}$ & $-156^{\circ} 19.6^{\prime}$ & 3181.6 & 8.1 & 103 & 1.4 & 263 & 9.4 & 100 & $\mathrm{C} / \mathrm{C}$ & & \\
\hline 10 & 12 & 1973 & 00 & $-12^{\circ} 22.3^{\prime}$ & $-156^{\circ} 7.5^{\prime}$ & 3193.7 & 8.1 & 103 & 1.4 & 263 & 9.4 & 100 & & & \\
\hline 10 & 12 & 1973 & 10 & $-12^{\circ} 24.1^{\prime}$ & $-155^{\circ} 59.4^{\prime}$ & 3201.8 & 8.6 & 101 & 0.8 & 275 & 9.4 & 100 & SATL & 0.50 & 7.9 \\
\hline 10 & 12 & 1973 & 130 & $-12^{\circ} 24.9^{\prime}$ & $-155^{\circ} 55.1^{\prime}$ & 3206.1 & 8.6 & $\begin{array}{r}101 \\
99\end{array}$ & 0.8 & 275 & 9.4 & 99 & $\mathrm{C} / \mathrm{C}$ & & \\
\hline 10 & 12 & 1973 & 352 & $-12^{\circ} 28.2^{\prime}$ & $-155^{\circ} 34.6^{\prime}$ & 3226.4 & 7.4 & 103 & 2.1 & 266 & 9.4 & 99 & SATL & 1.50 & 2.4 \\
\hline 10 & 12 & 1973 & 430 & $-12^{\circ} 29.2^{\prime}$ & $-155^{\circ} 29.9^{\prime}$ & 3231.1 & 7.4 & 110 & 2.1 & 266 & 9.4 & 105 & $\mathrm{C} / \mathrm{C}$ & & \\
\hline 10 & 12 & 1973 & 54 & $-12^{\circ} 30.7^{\prime}$ & $-155^{\circ} 25.9^{\prime}$ & 3235.3 & 8.1 & 108 & 1.3 & 265 & 9.4 & 105 & SATL & 0.50 & 2.6 \\
\hline 10 & 12 & 1973 & 650 & $-12^{\circ} 35.2^{\prime}$ & $-155^{\circ} 11.9^{\prime}$ & 3249.6 & 7.7 & 108 & 1.8 & 273 & 9.4 & 105 & SATL & 1.00 & 2.4 \\
\hline 10 & 12 & 1973 & 80 & $-12^{\circ} 37.9^{\prime}$ & $-155^{\circ} 3.1^{\prime}$ & 3258.6 & 7.7 & 104 & 1.8 & 273 & 9.4 & 102 & $\mathrm{C} / \mathrm{C}$ & & \\
\hline 10 & 12 & 1973 & 850 & $-12^{\circ} 39.5^{\prime}$ & $-154^{\circ} 56.8^{\prime}$ & 3265.0 & 7.7 & 103 & 1.7 & 278 & 9.4 & 102 & SATL & 1.50 & 3.6 \\
\hline 10 & 12 & 1973 & 1038 & $-12^{\circ} 42.6^{\prime}$ & $-154^{\circ} 42.9^{\prime}$ & 3278.9 & 7.6 & 103 & 1.8 & 279 & 9.4 & 102 & SATL & 0.25 & 3.1 \\
\hline 10 & 12 & 1973 & 1218 & $-12^{\circ} 45.4^{\prime}$ & $-154^{\circ} 30.2^{\prime}$ & 3291.6 & 7.5 & 105 & 1.9 & 271 & 9.4 & 102 & SATL & 1.50 & 3.0 \\
\hline 10 & 12 & 1973 & 1534 & $-12^{\circ} 51.7^{\prime}$ & $-154^{\circ} 59^{\prime}$ & 3316.1 & 7.8 & 105 & 1.7 & 266 & 9.4 & 102 & SATL & 1.50 & 6.4 \\
\hline 10 & 12 & 1973 & 1620 & $-12^{\circ} 53.3^{\prime}$ & $-154^{\circ} 0.0^{\prime}$ & 3322.1 & 7.7 & 101 & 1.7 & 266 & 9.4 & 98 & $\mathrm{C} / \mathrm{C}$ & & \\
\hline 10 & 12 & 1973 & 1650 & $-12^{\circ} 54.0^{\prime}$ & $-153^{\circ} 56.1^{\prime}$ & 3326.0 & 7.5 & 102 & 2.0 & 262 & 9.4 & 98 & SATL & 0.50 & 2.2 \\
\hline 10 & 12 & 1973 & 1828 & $-12^{\circ} 56.6^{\prime}$ & $-153^{\circ} 43.9^{\prime}$ & 3338.1 & 7.3 & 101 & 2.1 & 269 & 9.4 & $\begin{array}{l}70 \\
98\end{array}$ & SATL & 1.00 & 3.4 \\
\hline 10 & 12 & 1973 & 2020 & $-12^{\circ} 59.1^{\prime}$ & $-153^{\circ} 30.1^{\prime}$ & 3351.8 & 7.7 & 102 & $\begin{array}{l}2.1 \\
1.8\end{array}$ & 261 & 9.4 & 98 & SATL & 1.00 & 4.0 \\
\hline 10 & 12 & 1973 & 226 & $-13^{\circ} 1.9^{\prime}$ & $-153^{\circ} 164^{\prime}$ & 3365.5 & 7.9 & 102 & 1.6 & 259 & 9.4 & 98 & SATL & 0.50 & 3.2 \\
\hline 10 & 12 & 1973 & 235 & $-13^{\circ} 3.5^{\prime}$ & $-153^{\circ} 8.6^{\prime}$ & 3373.2 & 7.9 & 104 & 1.6 & 259 & 9.4 & 100 & $\mathrm{C} / \mathrm{C}$ & & \\
\hline 10 & 12 & 1973 & 2354 & $-13^{\circ} 5.1^{\prime}$ & $-153^{\circ} 2.2^{\prime}$ & 3379.7 & 7.8 & 104 & 1.7 & 261 & 9.4 & 100 & SATL & 0.50 & 3.0 \\
\hline 11 & 12 & 1973 & 00 & $-13^{\circ} 5.3^{\prime}$ & $-153^{\circ} 1.4^{\prime}$ & 3380.4 & 7.8 & 104 & 1.7 & 261 & 9.4 & 100 & & & \\
\hline 11 & 12 & 1973 & 34 & $-13^{\circ} 11.1^{\prime}$ & $-152^{\circ} 37.7^{\prime}$ & 3404.3 & 7.9 & 105 & 1.7 & 254 & 9.4 & 100 & SATL & 1.00 & 5.6 \\
\hline 11 & 12 & 1973 & 452 & $-13^{\circ} 14.9^{\prime}$ & $-152^{\circ} 23.6^{\prime}$ & 3418.5 & 7.9 & 102 & 1.6 & 268 & 9.4 & 100 & SATL & 0.50 & 3.1 \\
\hline 11 & 12 & 1973 & 550 & $-13^{\circ} 16.5^{\prime}$ & $-152^{\circ} 16.0^{\prime}$ & 3426.1 & 7.8 & 99 & 1.6 & 268 & 9.4 & 97 & C/C & & \\
\hline 11 & 12 & 1973 & 742 & $-13^{\circ} 18.8^{\prime}$ & $-152^{\circ} 1.1^{\prime}$ & 3440.8 & 7.8 & 100 & 1.7 & 263 & 9.4 & 97 & SATL & 1.00 & 4.5 \\
\hline 11 & 12 & 1973 & 84 & $-13^{\circ} 19.3^{\prime}$ & $-151^{\circ} 58.2^{\prime}$ & 3443.8 & 8.0 & 98 & 1.4 & 273 & 9.4 & 97 & SATL & 1.50 & 0.7 \\
\hline 11 & 12 & 1973 & 950 & $-13^{\circ} 21.2^{\prime}$ & $-151^{\circ} 43.8^{\prime}$ & 3457.8 & 8.0 & 97 & 1.4 & 278 & 9.4 & 97 & SATL & 1.50 & 2.5 \\
\hline 11 & 12 & 1973 & 1112 & $-13^{\circ} 22.5^{\prime}$ & $-151^{\circ} 32.7^{\prime}$ & 3468.6 & 7.9 & 99 & 1.6 & 265 & 9.4 & 97 & SATL & 1.00 & 2.0 \\
\hline 11 & 12 & 1973 & 1145 & $-13^{\circ} 23.2^{\prime}$ & $-151^{\circ} 28.3^{\prime}$ & 3473.0 & 7.9 & 102 & 1.6 & 265 & 9.4 & 99 & $\mathrm{C} / \mathrm{C}$ & & \\
\hline 11 & 12 & 1973 & 1152 & $-13^{\circ} 23.4^{\prime}$ & $-151^{\circ} 27.4^{\prime}$ & 3473.9 & 7.8 & 101 & 1.6 & 268 & 9.4 & 99 & SATL & 1.00 & 1.1 \\
\hline 11 & 12 & 1973 & 1446 & $-13^{\circ} 27.8^{\prime}$ & $-151^{\circ} 4.6^{\prime}$ & 3496.5 & 7.7 & 100 & 1.7 & 275 & 9.4 & 99 & SATL & 1.50 & 4.8 \\
\hline 11 & 12 & 1973 & 1634 & $-13^{\circ} 30.2^{\prime}$ & $-150^{\circ} 50.5^{\prime}$ & 3510.4 & 72 & 103 & 2.3 & 266 & 94 & 99 & SATL & 100 & 3.1 \\
\hline 11 & 12 & 1973 & 1736 & $-13^{\circ} 31.9^{\prime}$ & $-150^{\circ} 43.1^{\prime}$ & 3517.8 & 7.9 & 104 & 1.7 & 253 & 9.4 & 99 & SATL & 1.00 & 2.5 \\
\hline 11 & 12 & 1973 & 1926 & $-13^{\circ} 35.5^{\prime}$ & $-150^{\circ} 28.6^{\prime}$ & 3532.3 & 7.8 & 106 & 1.9 & 250 & 9.4 & 99 & SATL & 1.00 & 3.1 \\
\hline 11 & 12 & 1973 & 204 & $-13^{\circ} 36.8^{\prime}$ & $-150^{\circ} 23.7^{\prime}$ & 3537.3 & 7.7 & 102 & 1.9 & 250 & 9.4 & 96 & $\mathrm{C} / \mathrm{C}$ & & \\
\hline 11 & 12 & 1973 & 2116 & $-13^{\circ} 38.8^{\prime}$ & $-150^{\circ} 14.4^{\prime}$ & 3546.5 & 7.8 & 103 & 1.9 & 245 & 9.4 & 96 & SATL & 1.50 & 3.6 \\
\hline
\end{tabular}


TABLE 1 - Continued

\begin{tabular}{|c|c|c|c|c|c|c|c|c|c|c|c|c|c|c|c|c|c|}
\hline \multirow[b]{2}{*}{ DA } & \multirow[b]{2}{*}{ MO } & & & & & & & & Dri & & & & & & & & \\
\hline & & YR & Time & Latitude & Longitude & Dist. & Speed & Course & Speed & Hed. & Speed & Course & Comment & Qlty. & Dist. & Time & No. \\
\hline 11 & 12 & 1973 & 2210 & $-13^{\circ} 40.4^{\prime}$ & $\begin{array}{lll}-150^{\circ} & 7.3^{\prime}\end{array}$ & 3553.6 & 7.8 & 101 & 1.9 & 249 & 9.4 & 94 & $\mathrm{C} / \mathrm{C}$ & & & & 643 \\
\hline 11 & 12 & 1973 & 2250 & $-13^{\circ} 41.4^{\prime}$ & $-150^{\circ} \quad 2.1^{\prime}$ & 3558.8 & 7.8 & 100 & 1.8 & 249 & 9.4 & 94 & SATL & 1.00 & 3.0 & 1.6 & 645 \\
\hline 11 & 12 & 1973 & 2322 & $-13^{\circ} 42.1^{\prime}$ & $-149^{\circ} 57.9^{\prime}$ & 3562.9 & 7.8 & 99 & 1.8 & 249 & 9.4 & 94 & SATL & 1.00 & 1.0 & 0.5 & 647 \\
\hline 12 & 12 & 1973 & 00 & $-13^{\circ} 42.9^{\prime}$ & $-149^{\circ} 52.8^{\prime}$ & 3567.9 & 7.8 & 99 & 1.8 & 249 & 9.4 & 94 & & & & & 648 \\
\hline 12 & 12 & 1973 & 032 & $-13^{\circ} 43.6^{\prime}$ & $-149^{\circ} 48.6^{\prime}$ & 3572.1 & 7.7 & 97 & 1.8 & 259 & 9.4 & 94 & SATL & 0.50 & 2.1 & 1.2 & 650 \\
\hline 12 & 12 & 1973 & 214 & $-13^{\circ} 45.3^{\prime}$ & $-149^{\circ} 35.3^{\prime}$ & 3585.1 & 7.6 & 101 & 2.0 & 247 & 9.4 & 94 & SATL & 1.50 & 3.1 & 1.7 & 652 \\
\hline 12 & 12 & 1973 & 42 & $-13^{\circ} 47.9^{\prime}$ & $-149^{\circ} 21.4^{\prime}$ & 3598.8 & 7.9 & 104 & 2.1 & 235 & 9.4 & 94 & SATL & 1.00 & 3.7 & 1.8 & 654 \\
\hline 12 & 12 & 1973 & 650 & $-13^{\circ} 53.1^{\prime}$ & $-148^{\circ} 59.4^{\prime}$ & 3620.8 & 8.0 & 101 & 1.8 & 241 & 9.4 & 94 & SATL & 0.50 & 6.0 & 2.8 & 656 \\
\hline 12 & 12 & 1973 & 723 & $-13^{\circ} 53.9^{\prime}$ & $-148^{\circ} 55.0^{\prime}$ & 3625.2 & 7.9 & 96 & 1.8 & 241 & 9.4 & 90 & $\mathrm{C} / \mathrm{C}$ & & & & 657 \\
\hline 12 & 12 & 1973 & 90 & $-13^{\circ} 55.3^{\prime}$ & $-148^{\circ} 41.9^{\prime}$ & 3638.0 & 7.3 & 95 & 2.2 & 252 & 9.4 & 90 & SATL & 1.50 & 3.9 & 2.2 & 659 \\
\hline 12 & 12 & 1973 & 1150 & $-13^{\circ} 57.2^{\prime}$ & $-148^{\circ} 20.6^{\prime}$ & 3658.7 & 7.6 & 91 & 1.8 & 264 & 9.4 & 90 & SATL & 1.50 & 6.3 & 2.8 & 661 \\
\hline 12 & 12 & 1973 & 137 & $-13^{\circ} 57.4^{\prime}$ & $-148^{\circ} 10.5^{\prime}$ & 3668.5 & 7.6 & 95 & 1.8 & 264 & 9.4 & 93 & $\mathrm{C} / \mathrm{C}$ & & & & 662 \\
\hline 12 & 12 & 1973 & 1544 & $-13^{\circ} 59.2^{\prime}$ & $-147^{\circ} 50.0^{\prime}$ & 3688.5 & 8.1 & 93 & 1.3 & 276 & 9.4 & 93 & SATL & 0.50 & 7.0 & 3.9 & 664 \\
\hline 12 & 12 & 1973 & 1625 & $-13^{\circ} 59.4^{\prime}$ & $-147^{\circ} 44.3^{\prime}$ & 3694.0 & 8.1 & 103 & 1.3 & 276 & 9.4 & 102 & $\mathrm{C} / \mathrm{C}$ & & & & 665 \\
\hline 12 & 12 & 1973 & 1640 & $-13^{\circ} 59.9^{\prime}$ & $-147^{\circ} 42.3^{\prime}$ & 3696.0 & 7.5 & 104 & 1.9 & 273 & 9.4 & 102 & SATL & 1.50 & 1.3 & 0.9 & 667 \\
\hline 12 & 12 & 1973 & 1830 & $-14^{\circ} \quad 3.3^{\prime}$ & $-147^{\circ} 28.5^{\prime}$ & 3709.8 & 7.6 & 103 & 1.8 & 279 & 9.4 & 102 & SATL & 0.25 & 3.5 & 1.8 & 669 \\
\hline 12 & 12 & 1973 & 2028 & $-14^{\circ} \quad 6.6^{\prime}$ & $-147^{\circ} 13.4^{\prime}$ & 3724.9 & 7.6 & 103 & 1.8 & 278 & 9.4 & 102 & SATL & 1.00 & 3.5 & 2.0 & 671 \\
\hline 12 & 12 & 1973 & 2155 & $-14^{\circ} 9.1^{\prime}$ & $-147^{\circ} 2.3^{\prime}$ & 3735.9 & 7.6 & 99 & 1.8 & 278 & 9.4 & 99 & $\mathrm{C} / \mathrm{C}$ & & & & 672 \\
\hline 12 & 12 & 1973 & 2232 & $-14^{\circ} \quad 9.8^{\prime}$ & $-146^{\circ} 57.5^{\prime}$ & 3740.6 & 7.9 & 99 & 1.5 & 279 & 9.4 & 99 & SATL & 1.00 & 3.7 & 2.1 & 674 \\
\hline 12 & 12 & 1973 & 2326 & $-14^{\circ} 10.9^{\prime}$ & $-146^{\circ} 50.3^{\prime}$ & 3747.7 & 7.8 & 101 & 1.7 & 269 & 9.4 & 99 & SATL & 0.50 & 1.4 & 0.9 & 676 \\
\hline 12 & 12 & 1973 & 2356 & $-14^{\circ} 11.7^{\prime}$ & $-146^{\circ} 46.4^{\prime}$ & 3751.6 & 7.8 & 108 & 1.7 & 269 & 9.4 & 105 & $\mathrm{C} / \mathrm{C}$ & & & & 677 \\
\hline 13 & 12 & 1973 & 00 & $-14^{\circ} 11.8^{\prime}$ & $-146^{\circ} 45.8^{\prime}$ & 3752.1 & 7.8 & 108 & 1.7 & 269 & 9.4 & 105 & & & & & 678 \\
\hline 13 & 12 & 1973 & 20 & $-14^{\circ} 16.8^{\prime}$ & $-146^{\circ} 30.5^{\prime}$ & 3767.7 & 10.5 & 222 & 1.7 & 269 & 9.4 & 215 & $\mathrm{C} / \mathrm{C}$ & & & & 679 \\
\hline 13 & 12 & 1973 & 222 & $-14^{\circ} 19.6^{\prime}$ & $-146^{\circ} 33.2^{\prime}$ & 3771.6 & 10.4 & 220 & 1.7 & 269 & 9.4 & 212 & $\mathrm{C} / \mathrm{C}$ & & & & 680 \\
\hline 13 & 12 & 1973 & 46 & $-14^{\circ} 33.5^{\prime}$ & $-146^{\circ} 45.1^{\prime}$ & 3789.6 & 9.4 & 215 & 0.4 & 303 & 9.4 & 212 & SATL & 1.00 & 7.8 & 4.7 & 682 \\
\hline 13 & 12 & 1973 & 426 & $-14^{\circ} 36.1^{\prime}$ & $-146^{\circ} 46.9^{\prime}$ & 3792.7 & 9.3 & 195 & 0.4 & 303 & 9.4 & 193 & $\mathrm{C} / \mathrm{C}$ & & & & 683 \\
\hline 13 & 12 & 1973 & 440 & $-14^{\circ} 38.2^{\prime}$ & $-146^{\circ} 47.5^{\prime}$ & 3794.9 & 9.3 & 198 & 0.4 & 303 & 9.4 & 196 & $\mathrm{C} / \mathrm{C}$ & & & & 684 \\
\hline 13 & 12 & 1973 & 516 & $-14^{\circ} 43.4^{\prime}$ & $-146^{\circ} 49.3^{\prime}$ & 3800.5 & 8.4 & 199 & 0.4 & 303 & 8.5 & 196 & $\mathrm{C} / \mathrm{S}$ & & & & 685 \\
\hline 13 & 12 & 1973 & 558 & $-14^{\circ} 49.0^{\prime}$ & $-146^{\circ} 51.3^{\prime}$ & 3806.3 & 8.8 & 196 & 0.3 & 204 & 8.5 & 196 & SATL & 1.50 & 0.8 & 1.9 & 687 \\
\hline 13 & 12 & 1973 & 657 & $-14^{\circ} 57.3^{\prime}$ & $-146^{\circ} 53.8^{\prime}$ & 3815.0 & 8.2 & 5 & 0.3 & 204 & 8.5 & 6 & $\mathrm{C} / \mathrm{C}$ & & & & 688 \\
\hline 13 & 12 & 1973 & 722 & $-14^{\circ} 54.0^{\prime}$ & $-146^{\circ} 53.5^{\prime}$ & 3838.4 & 8.2 & 24 & 0.3 & 204 & 8.5 & 24 & $\mathrm{C} / \mathrm{C}$ & & & & 689 \\
\hline 13 & 12 & 1973 & 757 & $-14^{\circ} 49.6^{\prime}$ & $-146^{\circ} 51.5^{\prime}$ & 3823.2 & 8.5 & 24 & 0.0 & 0 & 8.5 & 24 & S318 & 1.50 & 0.7 & 2.0 & 691 \\
\hline 13 & 12 & 1973 & 757 & $-14^{\circ} 49.6^{\prime}$ & $-146^{\circ} 51.5^{\prime}$ & 3823.2 & 0.0 & 0 & 0.0 & 0 & 0.0 & 500 & STOP & & & & 692 \\
\hline 16 & 12 & 1973 & 1345 & $-14^{\circ} 49.6^{\prime}$ & $-146^{\circ} 51.5^{\prime}$ & 3823.2 & 0.7 & 224 & 0.7 & 224 & 0.0 & 500 & DEP & 1.50 & 0.1 & 77.8 & 694 \\
\hline 16 & 12 & 1973 & 1345 & $-14^{\circ} 49.6^{\prime}$ & $-146^{\circ} 51.5^{\prime}$ & 3823.2 & 8.3 & 218 & 0.7 & 224 & 7.6 & 217 & $\mathrm{U} / \mathrm{W}$ & & & & 695 \\
\hline 16 & 12 & 1973 & 146 & $-14^{\circ} 51.9^{\prime}$ & $-146^{\circ} 53.3^{\prime}$ & 3826.1 & 10.1 & 206 & 0.7 & 224 & 9.4 & 205 & $\mathrm{C} / \mathrm{CS}$ & & & & 696 \\
\hline 16 & 12 & 1973 & 1416 & $-14^{\circ} 53.4^{\prime}$ & $-146^{\circ} 54.1^{\prime}$ & 3827.8 & 9.2 & 203 & 0.3 & 79 & 9.4 & 205 & SATL & 1.50 & 0.4 & 0.5 & 698 \\
\hline 16 & 12 & 1973 & 166 & $-15^{\circ} 8.9^{\prime}$ & $\begin{array}{lll}-147^{\circ} & 1.0^{\prime}\end{array}$ & 3844.7 & 9.0 & 207 & 0.5 & 351 & 9.4 & 205 & SATL & 1.00 & 0.7 & 1.8 & 700 \\
\hline 16 & 12 & 1973 & 1721 & $-15^{\circ} 18.9^{\prime}$ & $-147^{\circ} \quad 6.3^{\prime}$ & 3855.9 & 9.0 & 219 & 0.5 & 351 & 9.4 & 217 & $\mathrm{C} / \mathrm{C}$ & & & & 701 \\
\hline 16 & 12 & 1973 & 1824 & $-15^{\circ} 26.2^{\prime}$ & $-147^{\circ} 12.5^{\prime}$ & 3865.3 & 9.4 & 216 & 0.2 & 144 & 9.4 & 217 & SATL & 0.00 & 1.3 & 2.3 & 703 \\
\hline 16 & 12 & 1973 & 1828 & $-15^{\circ} 26.7^{\prime}$ & $-147^{\circ} 12.9^{\prime}$ & 3866.0 & 9.4 & 243 & 0.2 & 144 & 9.4 & 244 & $\mathrm{C} / \mathrm{C}$ & & & & 704 \\
\hline 16 & 12 & 1973 & 2152 & $-15^{\circ} 41.2^{\prime}$ & $-147^{\circ} 42.4^{\prime}$ & 3897.8 & 9.4 & 251 & 0.2 & 144 & 9.4 & 252 & $\mathrm{C} / \mathrm{C}$ & & & & 705 \\
\hline 16 & 12 & 1973 & 2232 & $-15^{\circ} 43.2^{\prime}$ & $-147^{\circ} 48.5^{\prime}$ & 3904.1 & 9.4 & 251 & 0.2 & 157 & 9.4 & 252 & SATL & 1.00 & 0.7 & 4.0 & 707 \\
\hline 16 & 12 & 1973 & 2322 & $-15^{\circ} 45.8^{\prime}$ & $-147^{\circ} 56.2^{\prime}$ & 3911.9 & 9.3 & 263 & 0.2 & 157 & 9.4 & 264 & $\mathrm{C} / \mathrm{C}$ & & & & 708 \\
\hline 16 & 12 & 1973 & 1252 & $-15^{\circ} 46.3^{\prime}$ & $-148^{\circ} \quad 1.0^{\prime}$ & 3916.6 & 9.4 & 258 & 0.2 & 157 & 9.4 & 259 & $\mathrm{C} / \mathrm{C}$ & & & & 709 \\
\hline 17 & 12 & 1973 & 00 & $-15^{\circ} 46.6^{\prime}$ & $-148^{\circ} \quad 2.3^{\prime}$ & 3917.8 & 9.4 & 258 & 0.2 & 157 & 9.4 & 259 & & & & & 710 \\
\hline 17 & 12 & 1973 & 018 & $-15^{\circ} 47.2^{\prime}$ & $-148^{\circ} \quad 5.1^{\prime}$ & 3920.6 & 9.1 & 259 & 0.3 & 74 & 9.4 & 259 & SATL & 0.50 & 0.4 & 1.8 & 712 \\
\hline 17 & 12 & 1973 & 020 & $-15^{\circ} 47.3^{\prime}$ & $-148^{\circ} \quad 5.4^{\prime}$ & 3920.9 & 9.1 & 252 & 0.3 & 74 & 9.4 & 252 & $\mathrm{C} / \mathrm{C}$ & & & & 713 \\
\hline 17 & 12 & 1973 & 10 & $-15^{\circ} 49.1^{\prime}$ & $-148^{\circ} 11.4^{\prime}$ & 3927.0 & 9.2 & 291 & 0.3 & 74 & 9.4 & 290 & $\mathrm{C} / \mathrm{C}$ & & & & 714 \\
\hline 17 & 12 & 1973 & 111 & $-15^{\circ} 48.5^{\prime}$ & $-148^{\circ} 13.0^{\prime}$ & 3928.7 & 9.3 & 311 & 0.3 & 74 & 9.4 & 310 & $\mathrm{C} / \mathrm{C}$ & & & & 715 \\
\hline 17 & 12 & 1973 & 120 & $-15^{\circ} 47.6^{\prime}$ & $-148^{\circ} 14.1^{\prime}$ & 3930.1 & 9.2 & 301 & 0.3 & 74 & 9.4 & 300 & $\mathrm{C} / \mathrm{C}$ & & & & 716 \\
\hline 17 & 12 & 1973 & 123 & $-15^{\circ} 47.4^{\prime}$ & $-148^{\circ} 14.5^{\prime}$ & 3930.5 & 9.2 & 291 & 0.3 & 74 & 9.4 & 290 & $\mathrm{C} / \mathrm{C}$ & & & & 717 \\
\hline 17 & 12 & 1973 & 130 & $-15^{\circ} 47.0^{\prime}$ & $-148^{\circ} 15.6^{\prime}$ & 3931.6 & 9.1 & 270 & 0.3 & 74 & 9.4 & 270 & $\mathrm{C} / \mathrm{C}$ & & & & 718 \\
\hline 17 & 12 & 1973 & 135 & $-15^{\circ} 47.0^{\prime}$ & $-148^{\circ} 16.4^{\prime}$ & 3932.4 & 9.1 & 250 & 0.3 & 74 & 9.4 & 250 & $\mathrm{C} / \mathrm{C}$ & & & & 719 \\
\hline 17 & 12 & 1973 & 139 & $-15^{\circ} 47.2^{\prime}$ & $-148^{\circ} 17.0^{\prime}$ & 3933.0 & 9.2 & 229 & 0.3 & 74 & 9.4 & 230 & $\mathrm{C} / \mathrm{C}$ & & & & 720 \\
\hline 17 & 12 & 1973 & 146 & $-15^{\circ} 47.9^{\prime}$ & $-148^{\circ} 17.8^{\prime}$ & 3934.0 & 9.7 & 227 & 0.5 & 167 & 9.4 & 230 & SATL & 0.00 & 0.5 & 1.5 & 722 \\
\hline 17 & 12 & 1973 & 148 & $-15^{\circ} 48.1^{\prime}$ & $-148^{\circ} 18.0^{\prime}$ & 3934.3 & 9.8 & 215 & 0.5 & 167 & 9.4 & 217 & $\mathrm{C} / \mathrm{C}$ & & & & 723 \\
\hline 17 & 12 & 1973 & 336 & $-16^{\circ} \quad 2.6^{\prime}$ & $-148^{\circ} 28.4^{\prime}$ & 3951.9 & 8.8 & 215 & 0.7 & 57 & 9.4 & 217 & SATL & 0.00 & 1.1 & 1.8 & 725 \\
\hline 17 & 12 & 1973 & 42 & $-16^{\circ} \quad 5.7^{\prime}$ & $-148^{\circ} 30.7^{\prime}$ & 3055.7 & 9.2 & 215 & 0.4 & 97 & 9.4 & 217 & SATL & 0.50 & 0.3 & 0.4 & 727 \\
\hline 17 & 12 & 1973 & 420 & $-16^{\circ} \quad 8.0^{\prime}$ & $-148^{\circ} 32.3^{\prime}$ & 3958.5 & 8.6 & 215 & 0.4 & 97 & 8.8 & 217 & $\mathrm{C} / \mathrm{S}$ & & & & 728 \\
\hline 17 & 12 & 1973 & 546 & $-16^{\circ} 18.1^{\prime}$ & $-148^{\circ} 39.7^{\prime}$ & 3970.8 & 9.0 & 216 & 0.2 & 191 & 8.8 & 217 & SATL & 0.50 & 0.7 & 1.7 & 730 \\
\hline 17 & 12 & 1973 & 90 & $-16^{\circ} 41.6^{\prime}$ & $-148^{\circ} 57.7^{\prime}$ & 4000.0 & 9.0 & 222 & 0.2 & 191 & 8.8 & 223 & $\mathrm{C} / \mathrm{C}$ & & & & 731 \\
\hline 17 & 12 & 1973 & 1032 & $-16^{\circ} 51.8^{\prime}$ & $-149^{\circ} \quad 7.4^{\prime}$ & 4013.8 & 9.1 & 221 & 0.5 & 176 & 8.8 & 223 & SATL & 1.50 & 1.2 & 4.8 & 733 \\
\hline 17 & 12 & 1973 & 1118 & $-16^{\circ} 57.1^{\prime}$ & $-149^{\circ} 12.2^{\prime}$ & 4020.8 & 9.2 & 213 & 0.5 & 176 & 8.8 & 215 & $\mathrm{C} / \mathrm{C}$ & & & & 734 \\
\hline 17 & 12 & 1973 & 1132 & $-16^{\circ} 58.9^{\prime}$ & $-149^{\circ} 13.4^{\prime}$ & 4022.9 & 9.0 & 213 & 0.3 & 157 & 8.8 & 215 & SATL & 1.50 & 0.5 & 1.0 & 736 \\
\hline 17 & 12 & 1973 & 120 & $\begin{array}{ll}-17^{\circ} & 2.4^{\prime}\end{array}$ & $-149^{\circ} 15.8^{\prime}$ & 4027.1 & 9.0 & 209 & 0.3 & 157 & 8.8 & 211 & $\mathrm{C} / \mathrm{C}$ & & & & 737 \\
\hline 17 & 12 & 1973 & 1215 & $-17^{\circ} \quad 4.4^{\prime}$ & $-149^{\circ} 17.0^{\prime}$ & 4029.4 & 9.3 & 209 & 0.3 & 157 & 9.1 & 211 & $\mathrm{C} / \mathrm{S}$ & & & & 738 \\
\hline 17 & 12 & 1973 & 1222 & $-17^{\circ} \quad 5.3^{\prime}$ & $-149^{\circ} 17.5^{\prime}$ & 4030.4 & 9.0 & 211 & 0.1 & 22 & 9.1 & 211 & SATL & 0.00 & 0.3 & 0.8 & 740 \\
\hline 17 & 12 & 1973 & 136 & $-17^{\circ} 11.0^{\prime}$ & $-149^{\circ} 21.1^{\prime}$ & 4037.1 & 9.0 & 214 & 0.1 & 22 & 9.1 & 214 & $\mathrm{C} / \mathrm{C}$ & & & & 741 \\
\hline 17 & 12 & 1973 & 140 & $-17^{\circ} 17.7^{\prime}$ & $-149^{\circ} 25.8^{\prime}$ & 4045.2 & 5.7 & 214 & 0.1 & 22 & 5.8 & 214 & $\mathrm{C} / \mathrm{S}$ & & & & 742 \\
\hline 17 & 12 & 1973 & 1417 & $-17^{\circ} 19.0^{\prime}$ & $-149^{\circ} 26.8^{\prime}$ & 4046.8 & 8.7 & 214 & 0.1 & 22 & 8.8 & 214 & $\mathrm{C} / \mathrm{S}$ & & & & 743 \\
\hline 17 & 12 & 1973 & 1420 & $-17^{\circ} 19.4^{\prime}$ & $-149^{\circ} 27.0^{\prime}$ & 4047.2 & 8.7 & 221 & 0.1 & 22 & 8.8 & 221 & $\mathrm{C} / \mathrm{C}$ & & & & 744 \\
\hline 17 & 12 & 1973 & 1435 & $-17^{\circ} 21.0^{\prime}$ & $-149^{\circ} 28.5^{\prime}$ & 4049.4 & 8.7 & 227 & 0.1 & 22 & 8.8 & 227 & $\mathrm{C} / \mathrm{C}$ & & & & 745 \\
\hline 17 & 12 & 1973 & 1440 & $-17^{\circ} 21.5^{\prime}$ & $-149^{\circ} 29.1^{\prime}$ & 4050.1 & 8.7 & 227 & 0.0 & 0 & 8.8 & 227 & DR & 0.00 & 0.3 & 2.3 & 746 \\
\hline
\end{tabular}

Previous accum miles $\quad 0.000$ present accum dist. $\quad 4050.115$ cruise DSDP33GC record no. 746

//End of all jobs $20 \mathrm{Feb} .7401 .953 \mathrm{hrs}$.

//Job $02162 \quad 20$ Feb. 7401.977 hrs McConnell

//XEQ DTNAV FX 20 Feb. 7401.977 hrs 


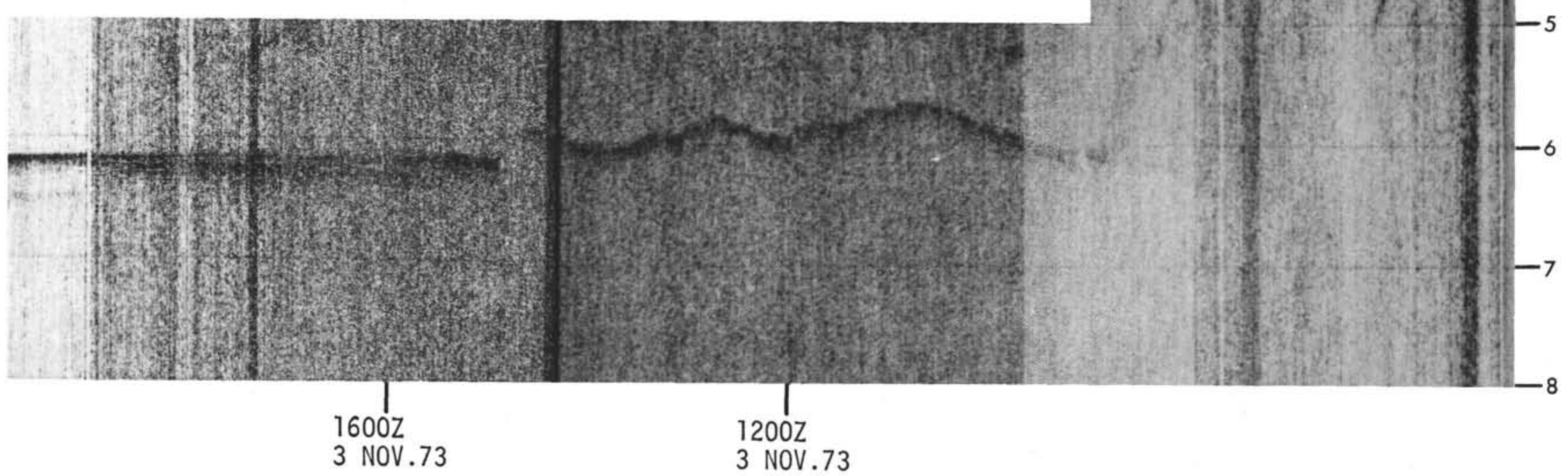

Figure 2. Seismic profiles from Leg 33. These are the photographs of the originals from the No. 1 EDO which was kept set for a 10-sec sweep. 

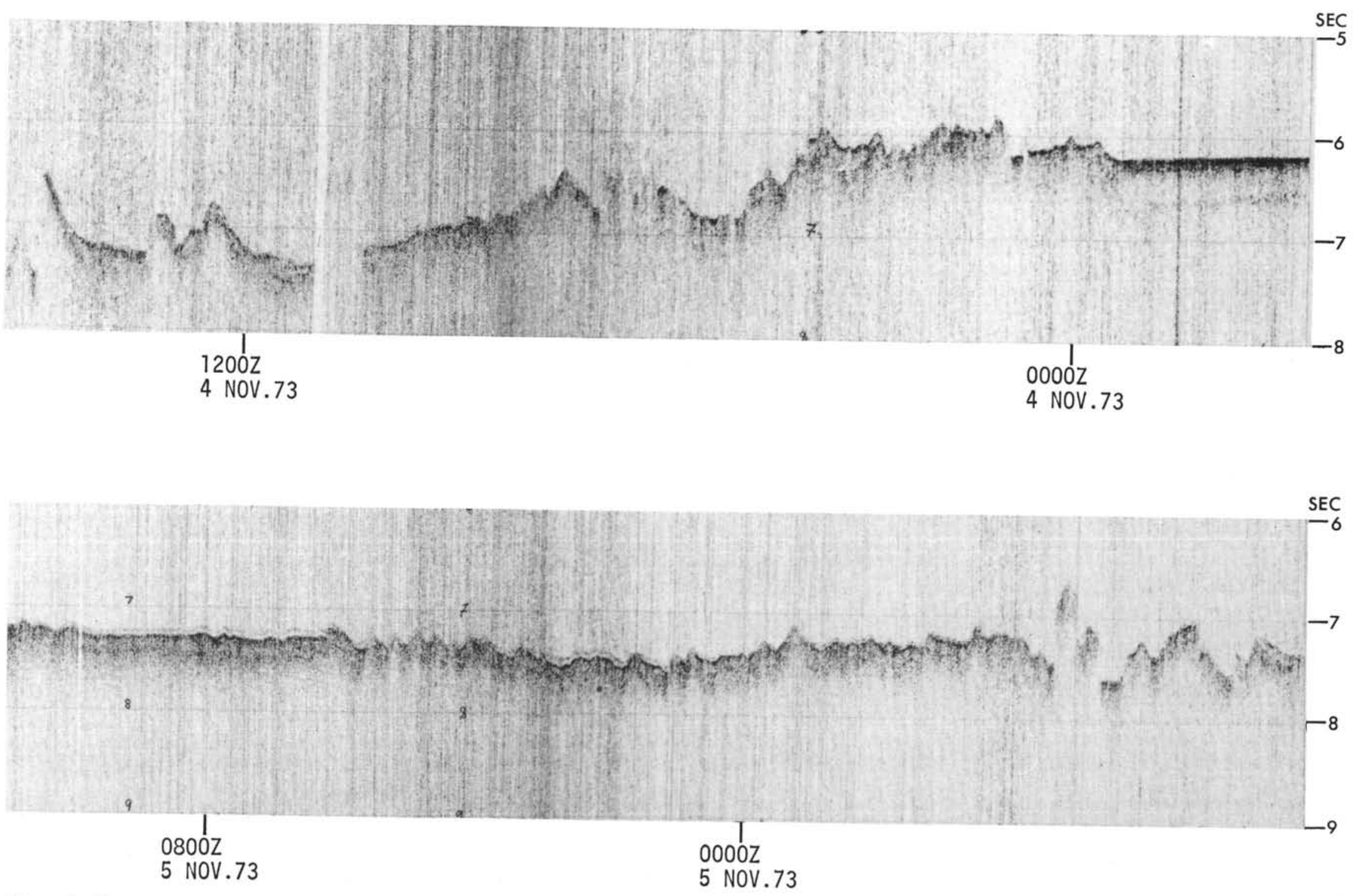

Figure 2. (Continued). 


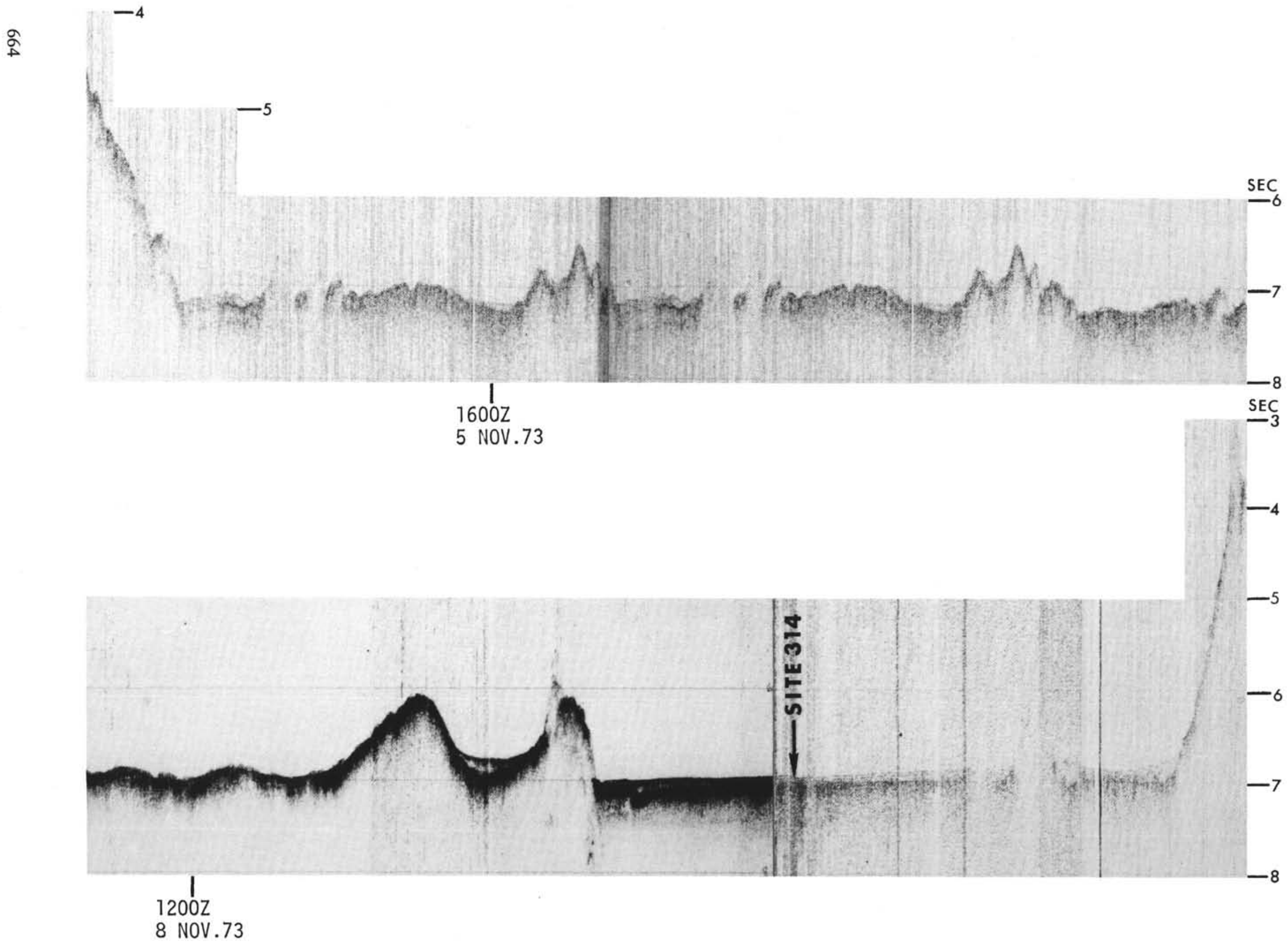

Figure 2. (Continued). 
SEC

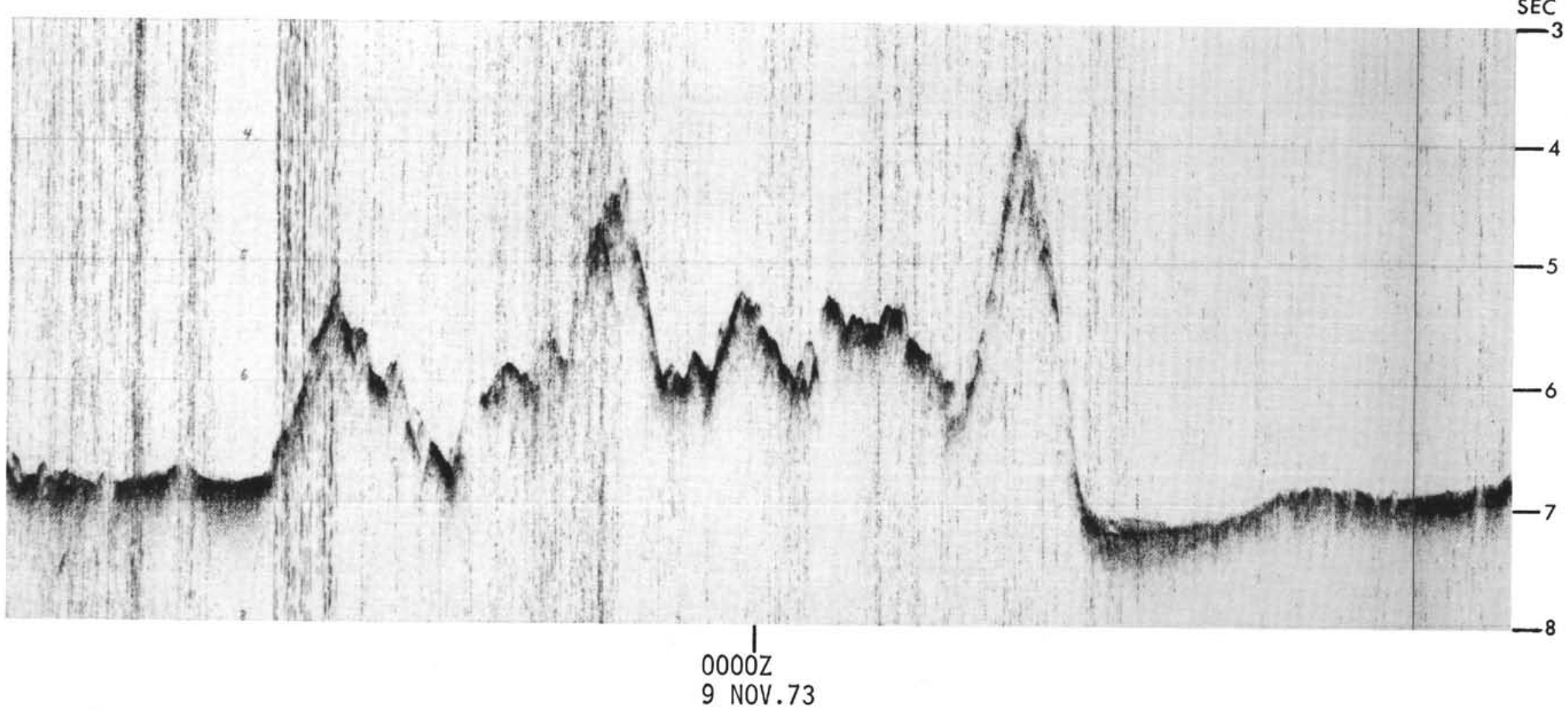

Figure 2. (Continued). 
ลे

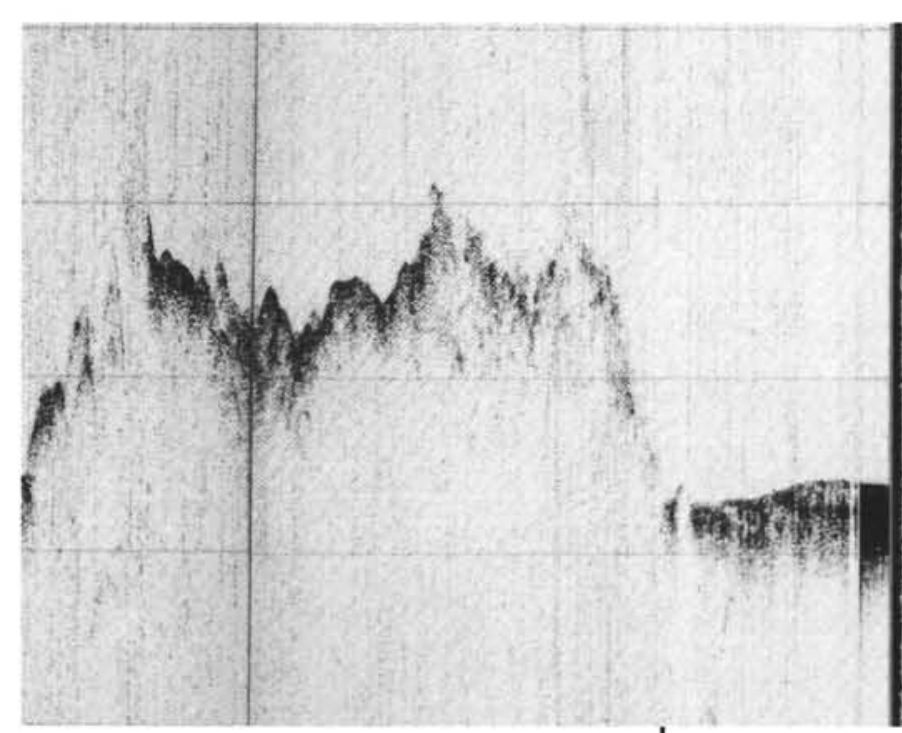

$0000 \mathrm{z}$

10 NOV.73

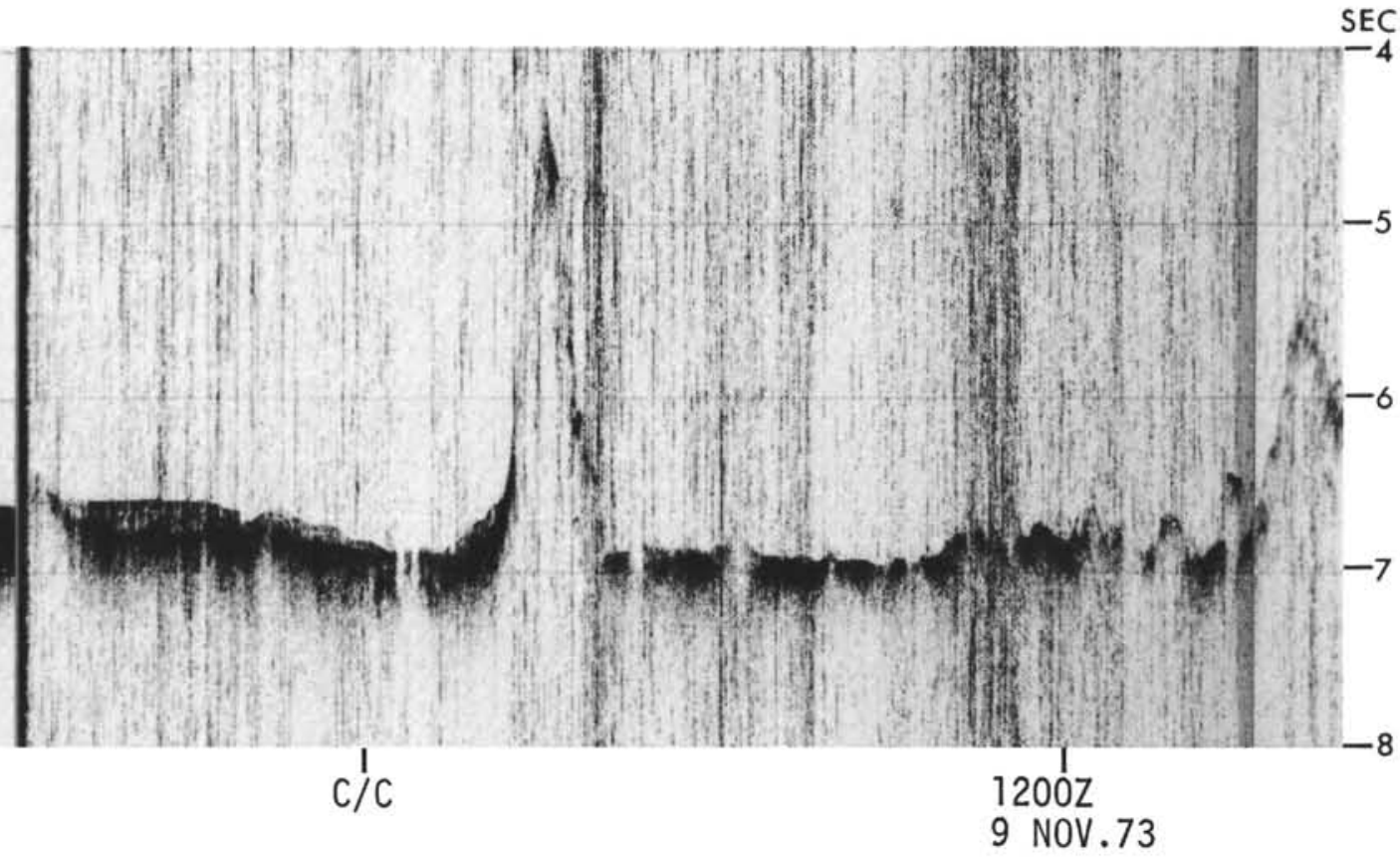

SEC

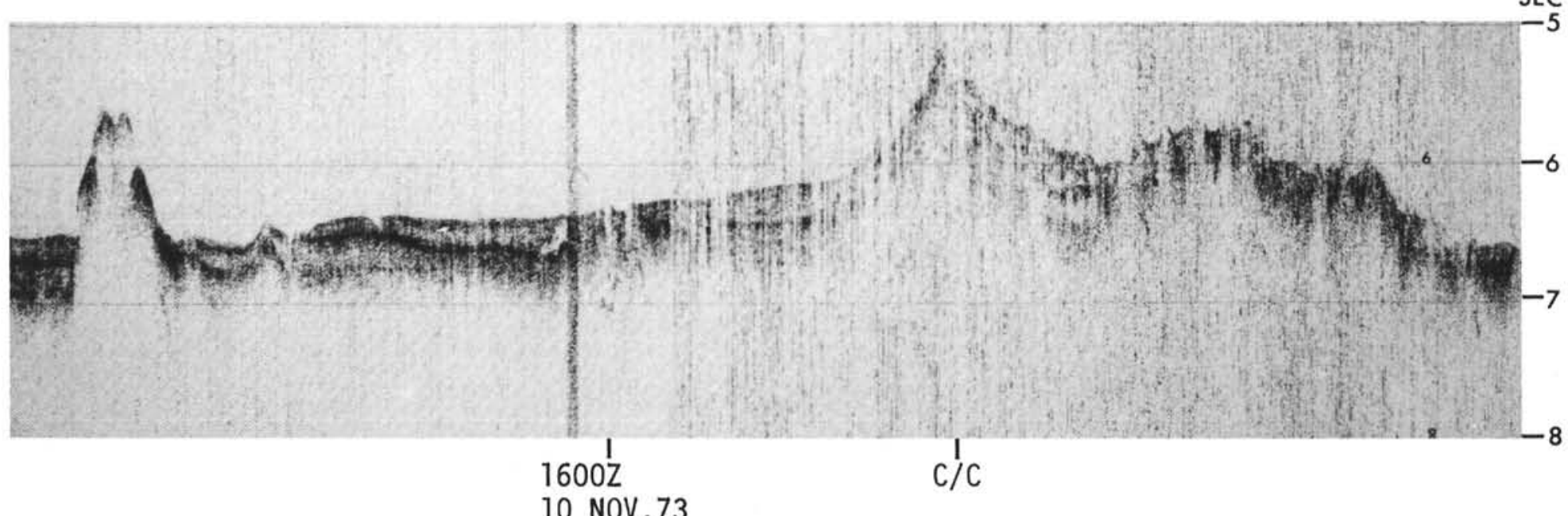

Figure 2. (Continued). 


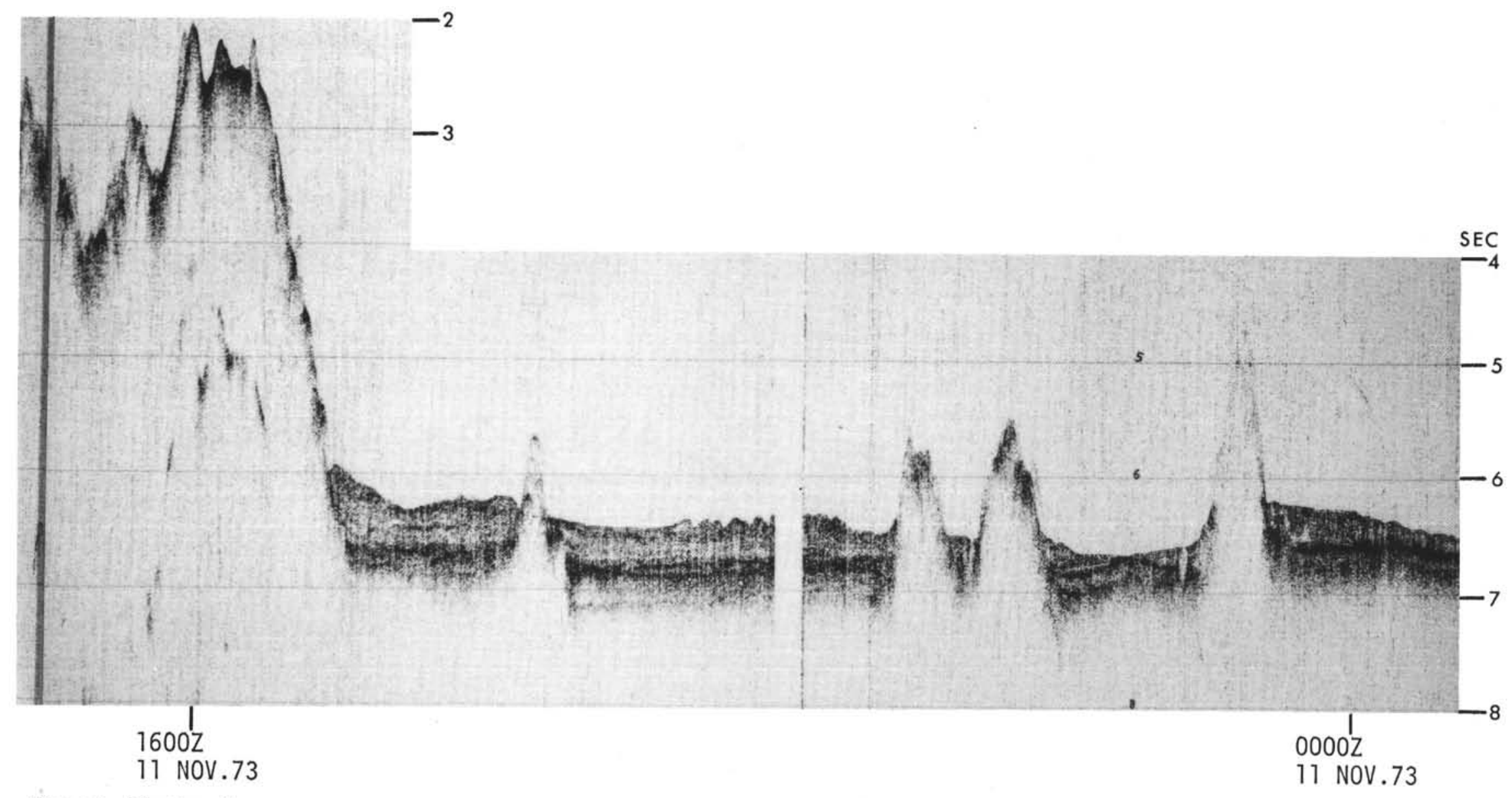

Figure 2. (Continued). 


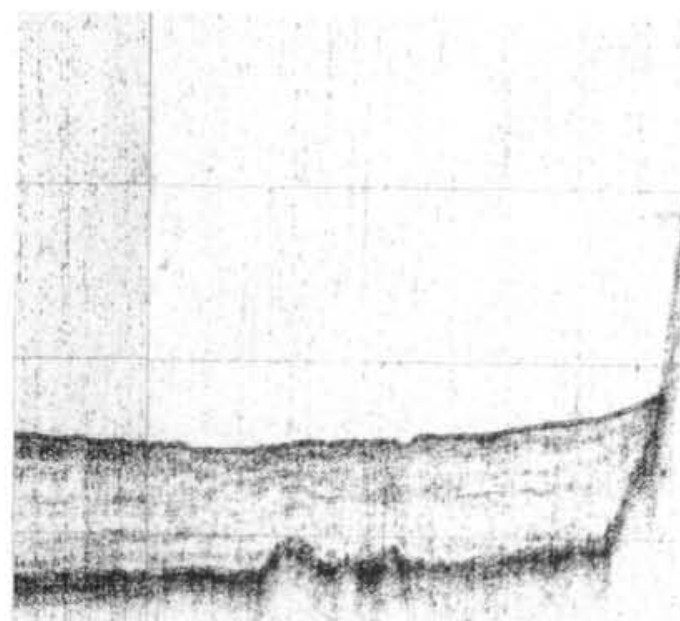

12002

12 NOV.73

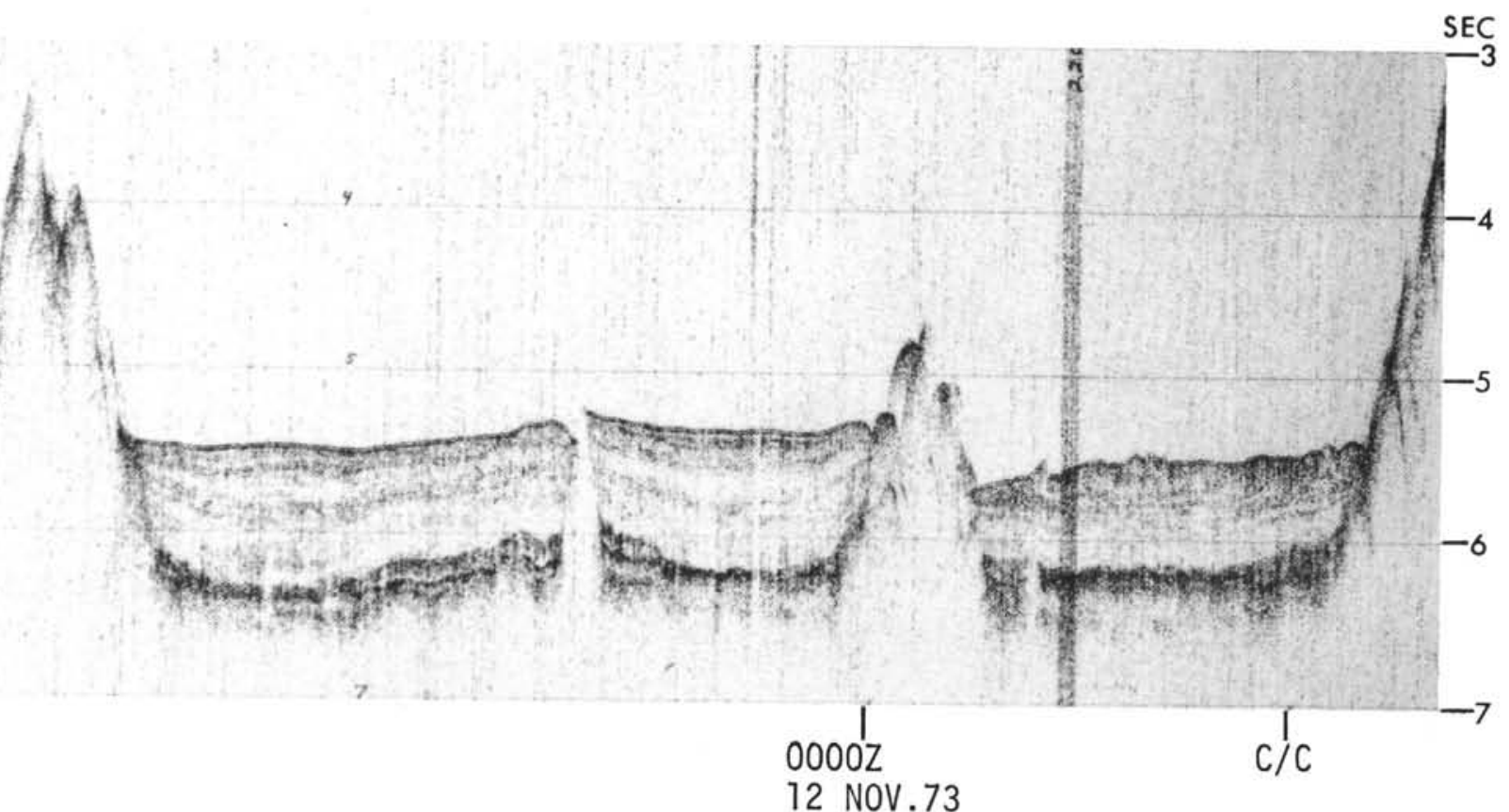

SEC

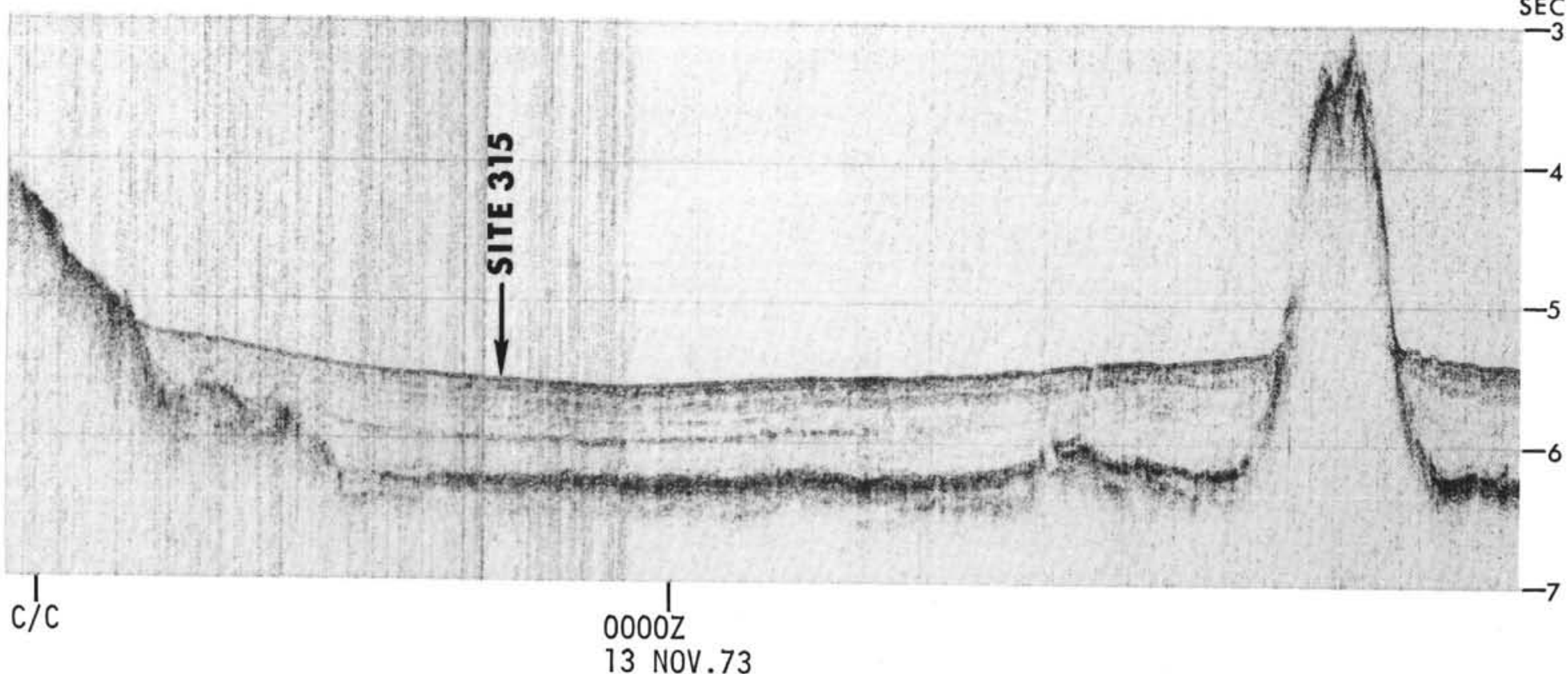




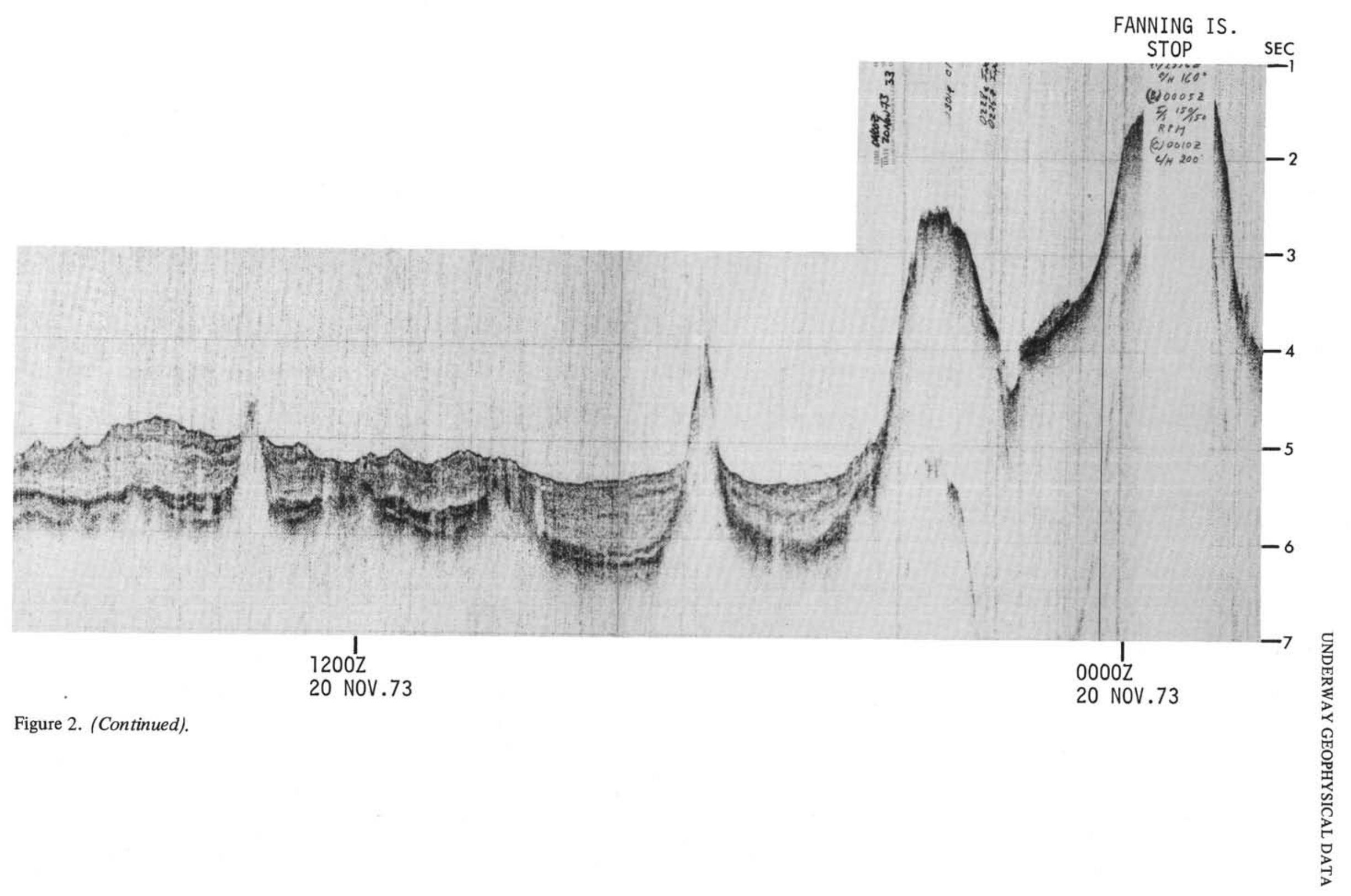




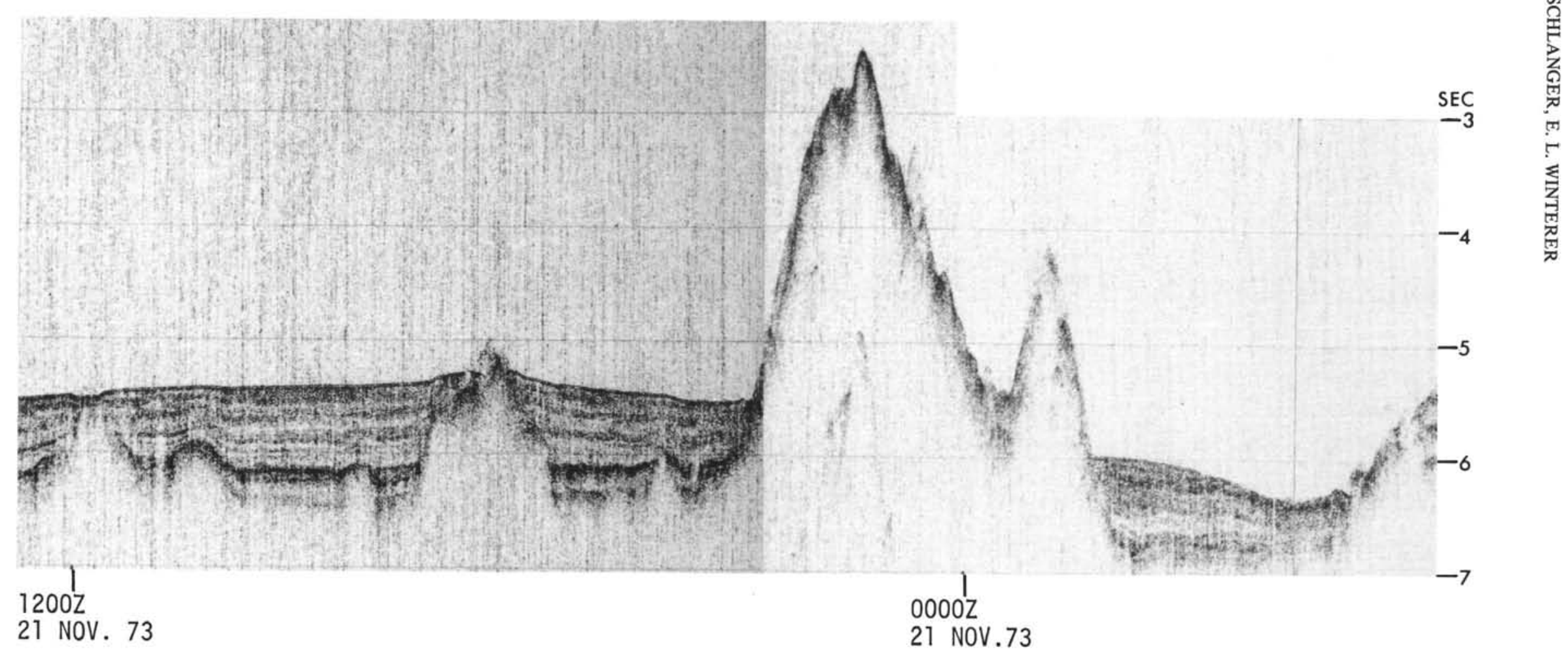

SEC

SITE 316

1.

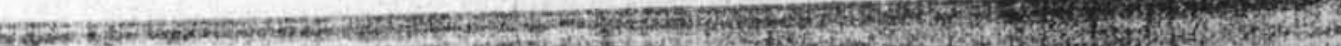

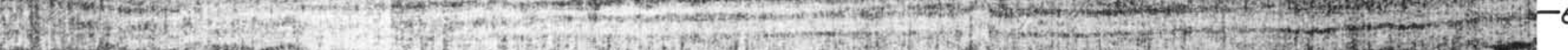

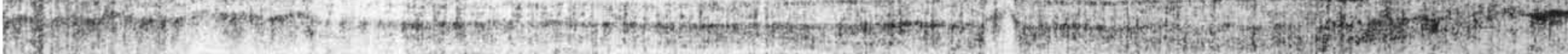
(3)

\section{0}

22 NOV.73

Figure 2. (Continued) 


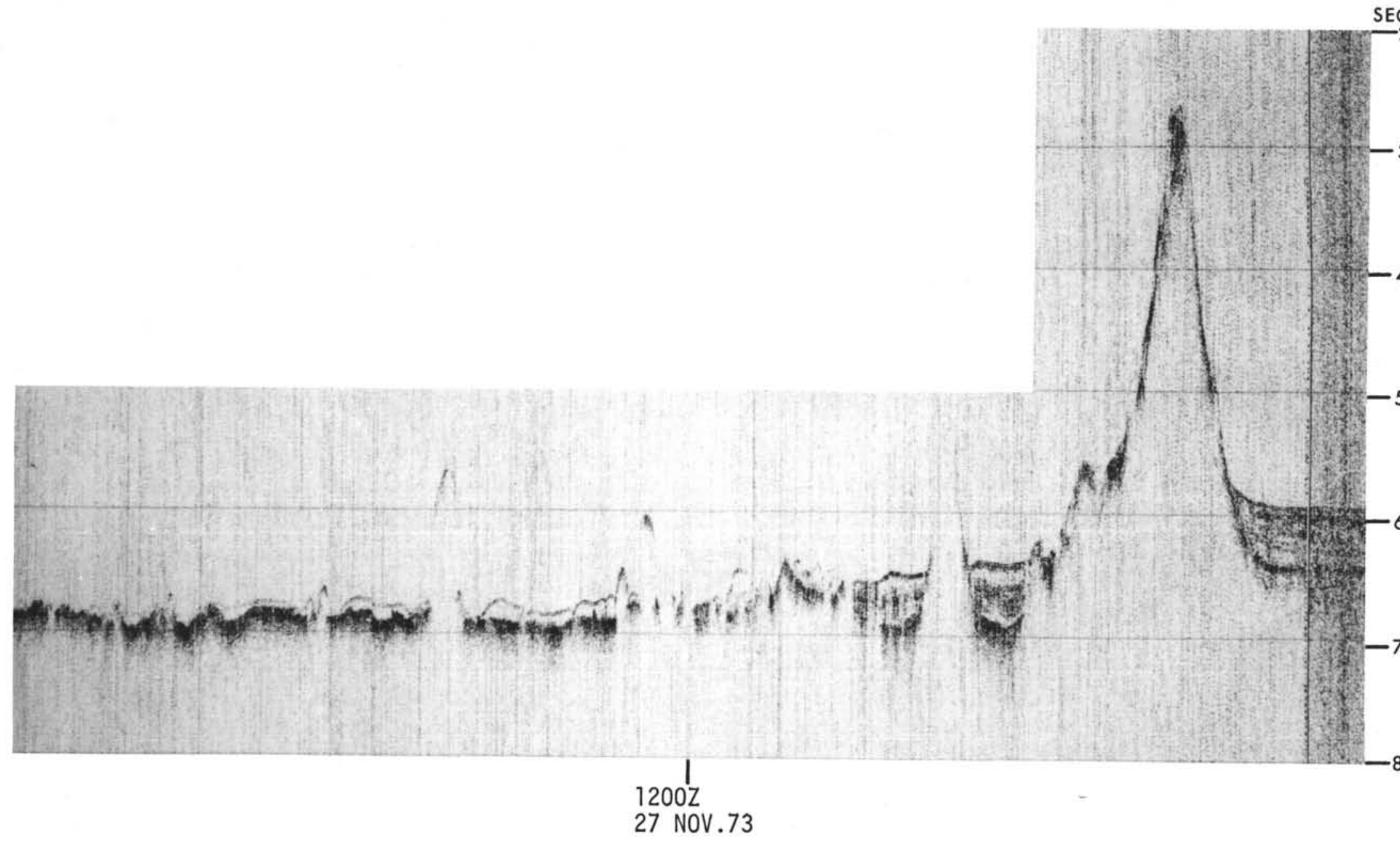

Figure 2. (Continued). 


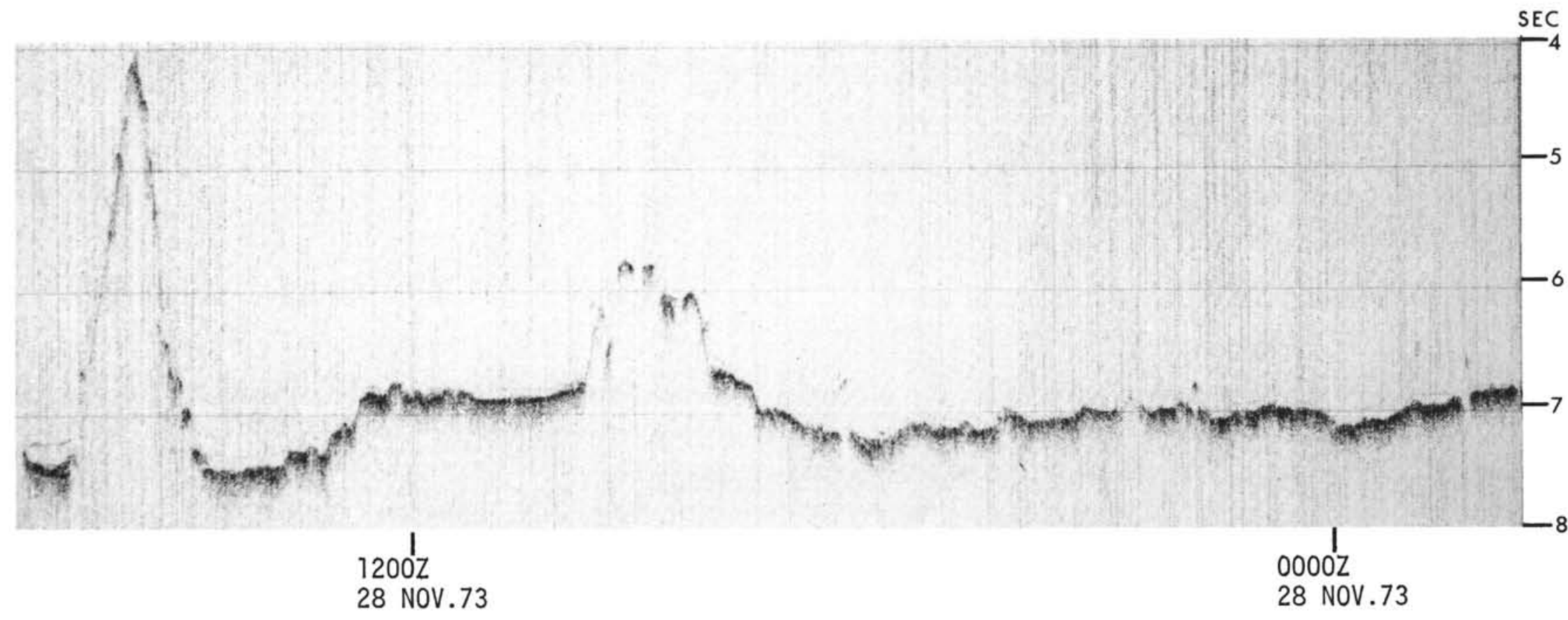

Figure 2. (Continued). 


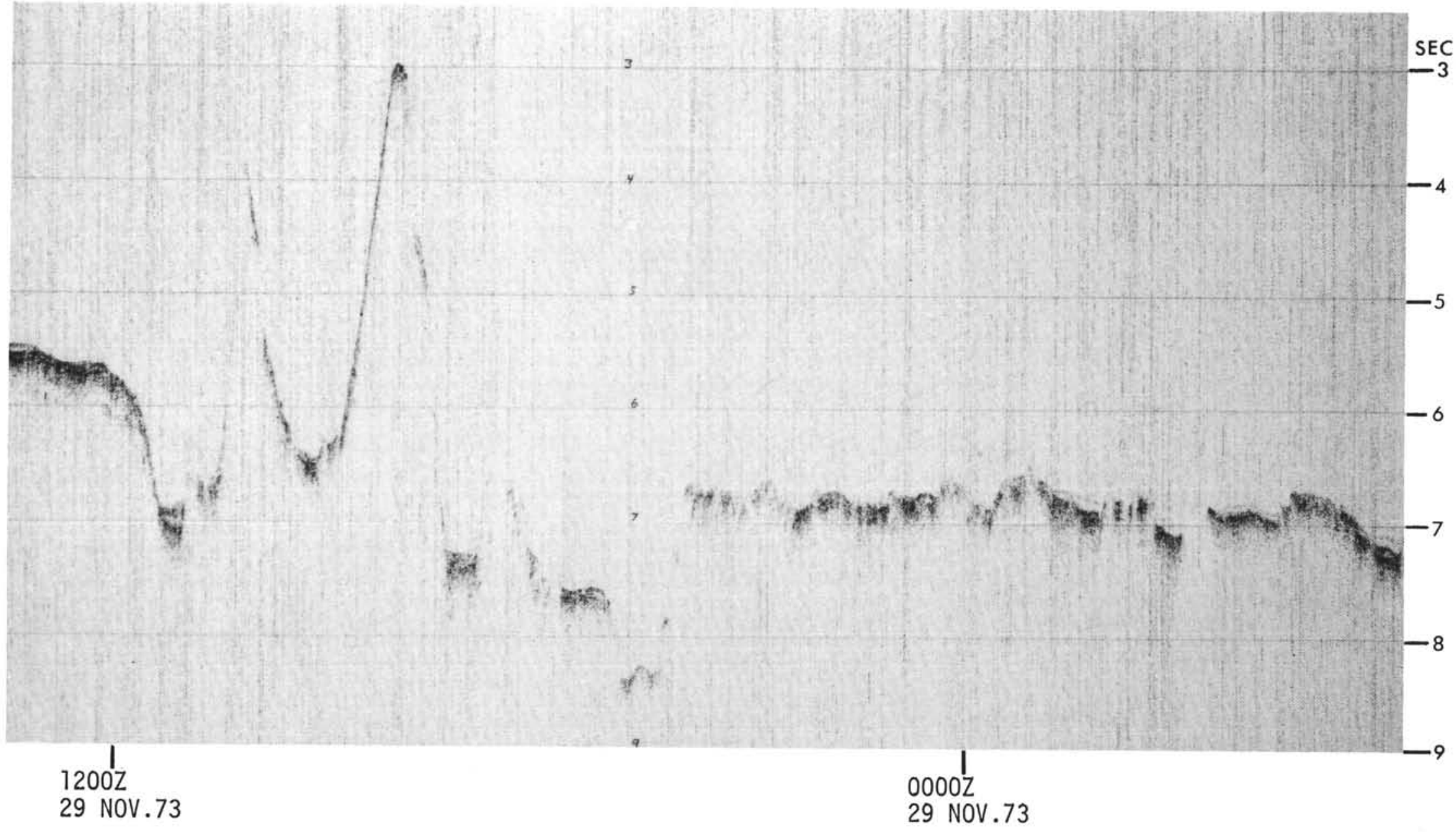

Figure 2. (Continued) 


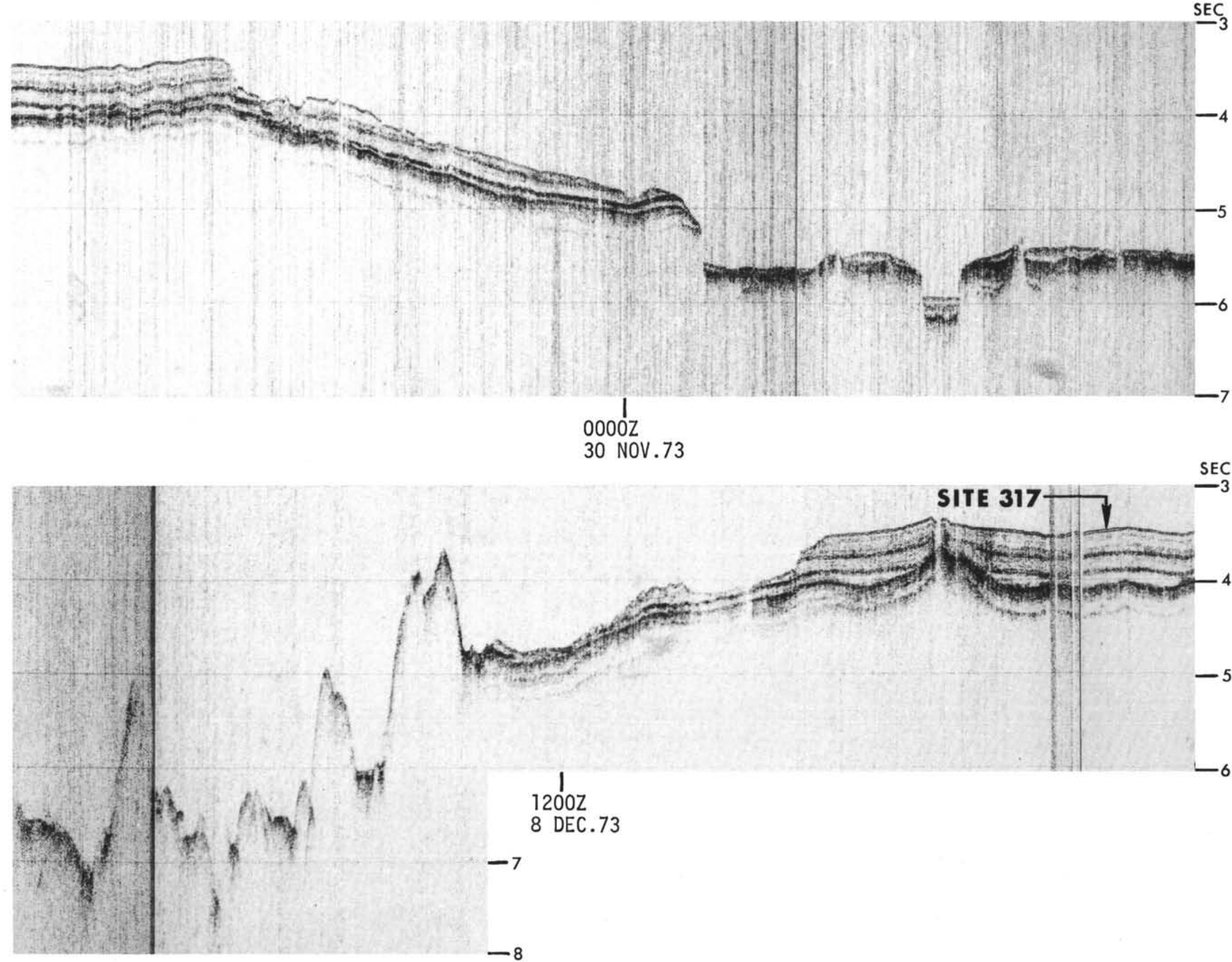

Figure 2. (Continued) 

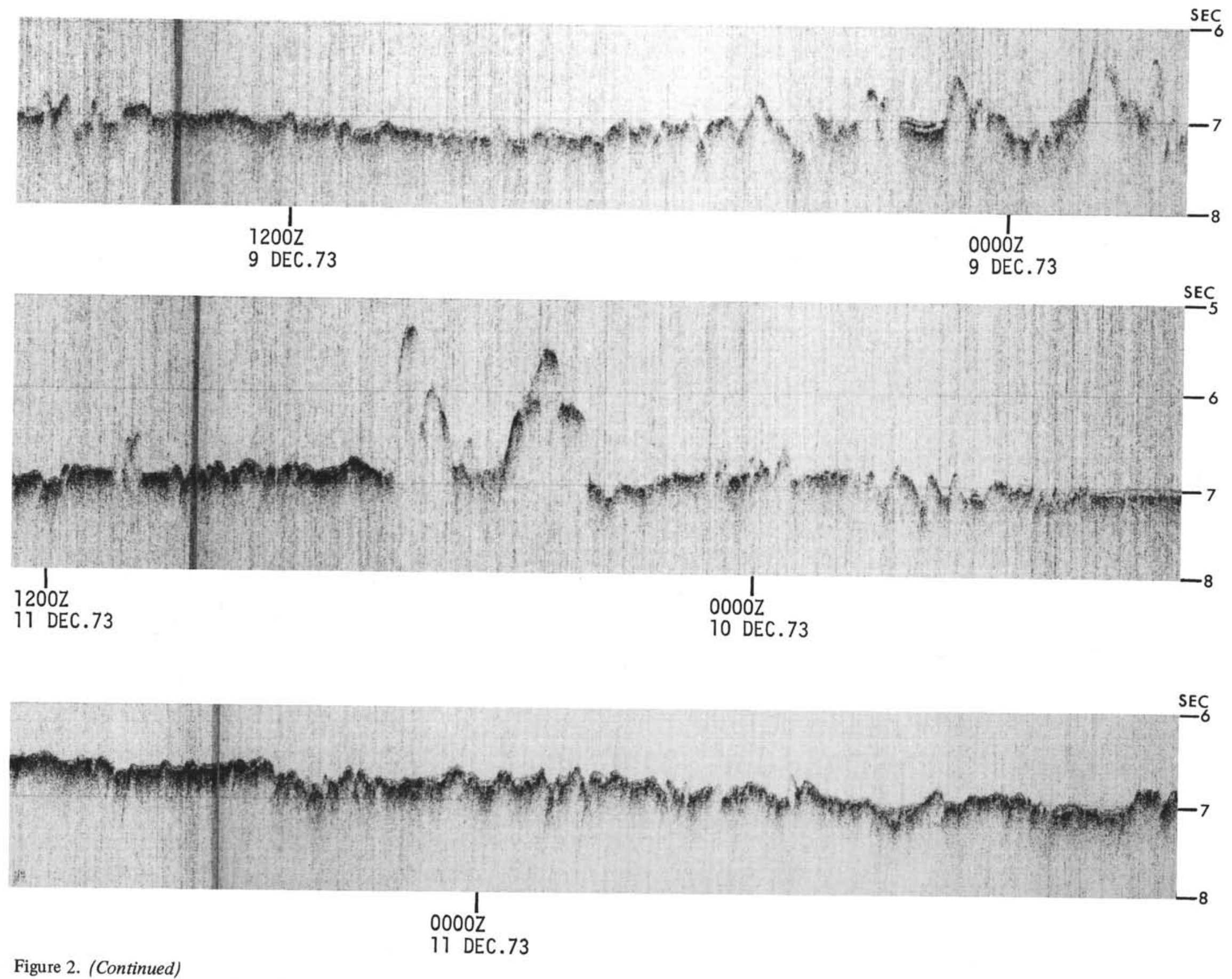

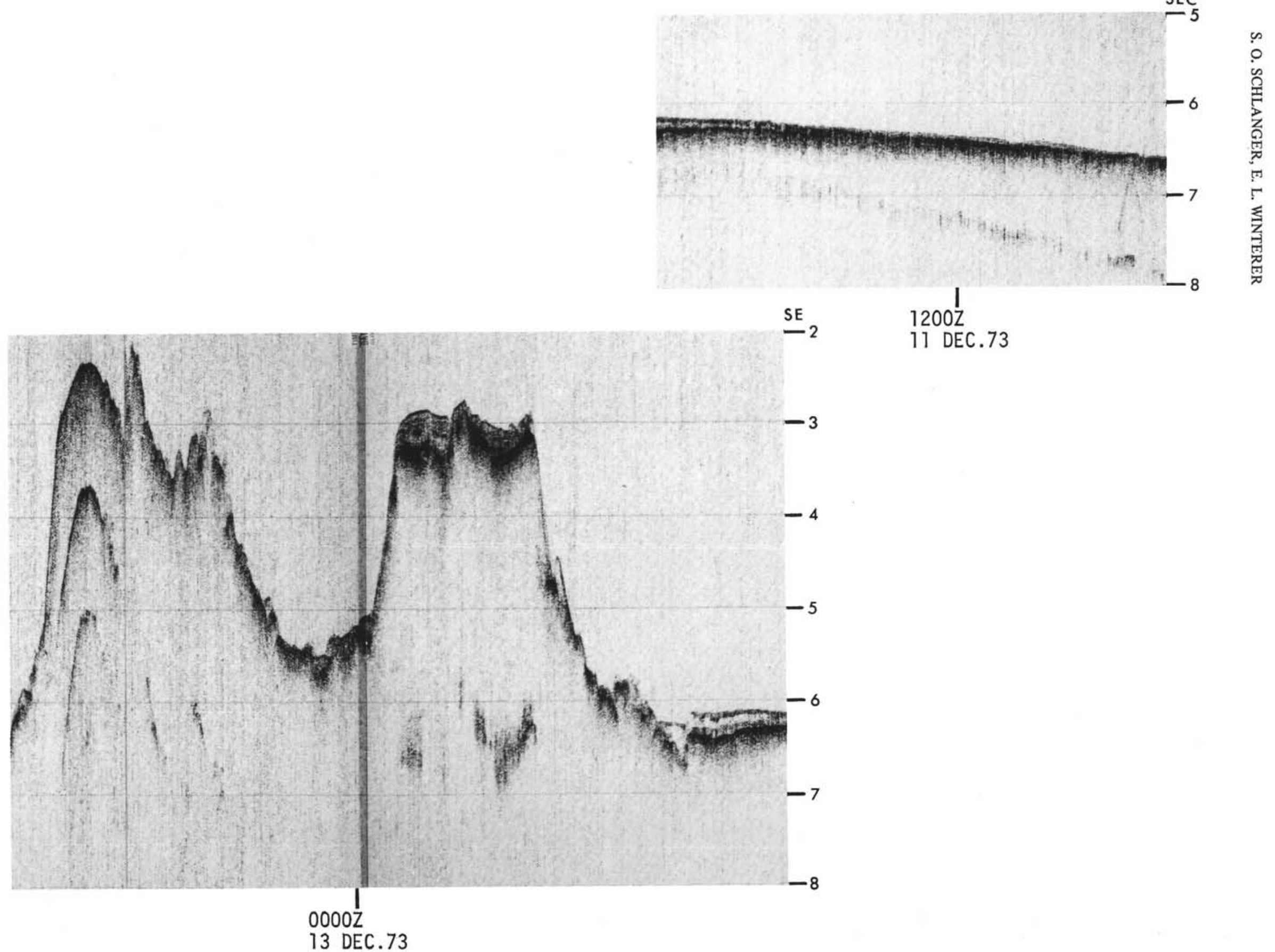

Figure 2. (Continued) 


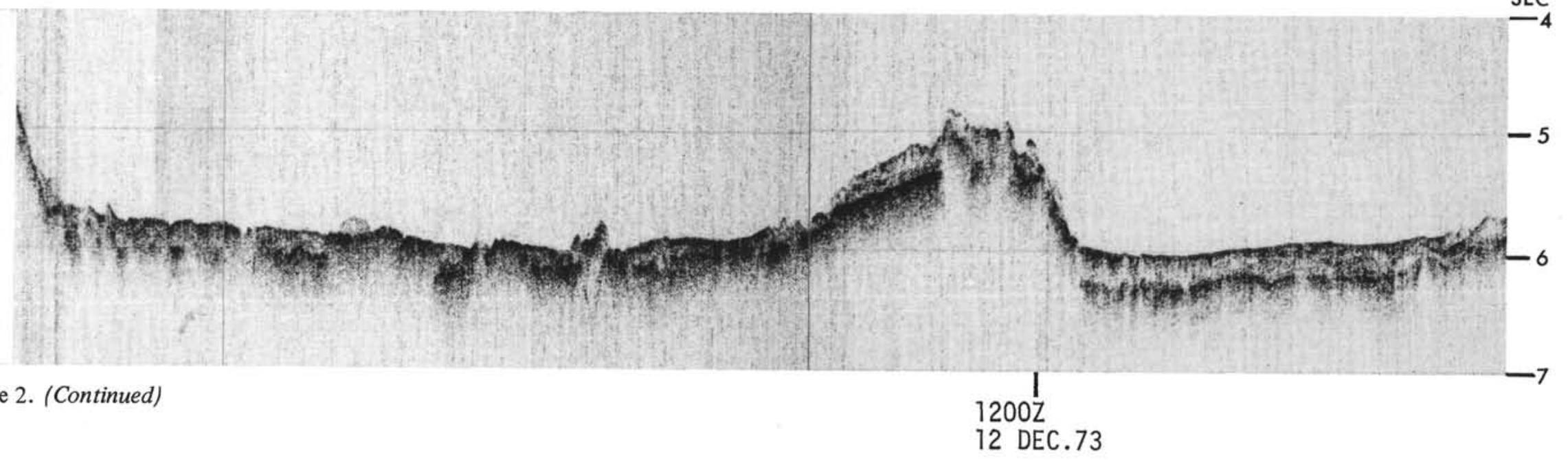




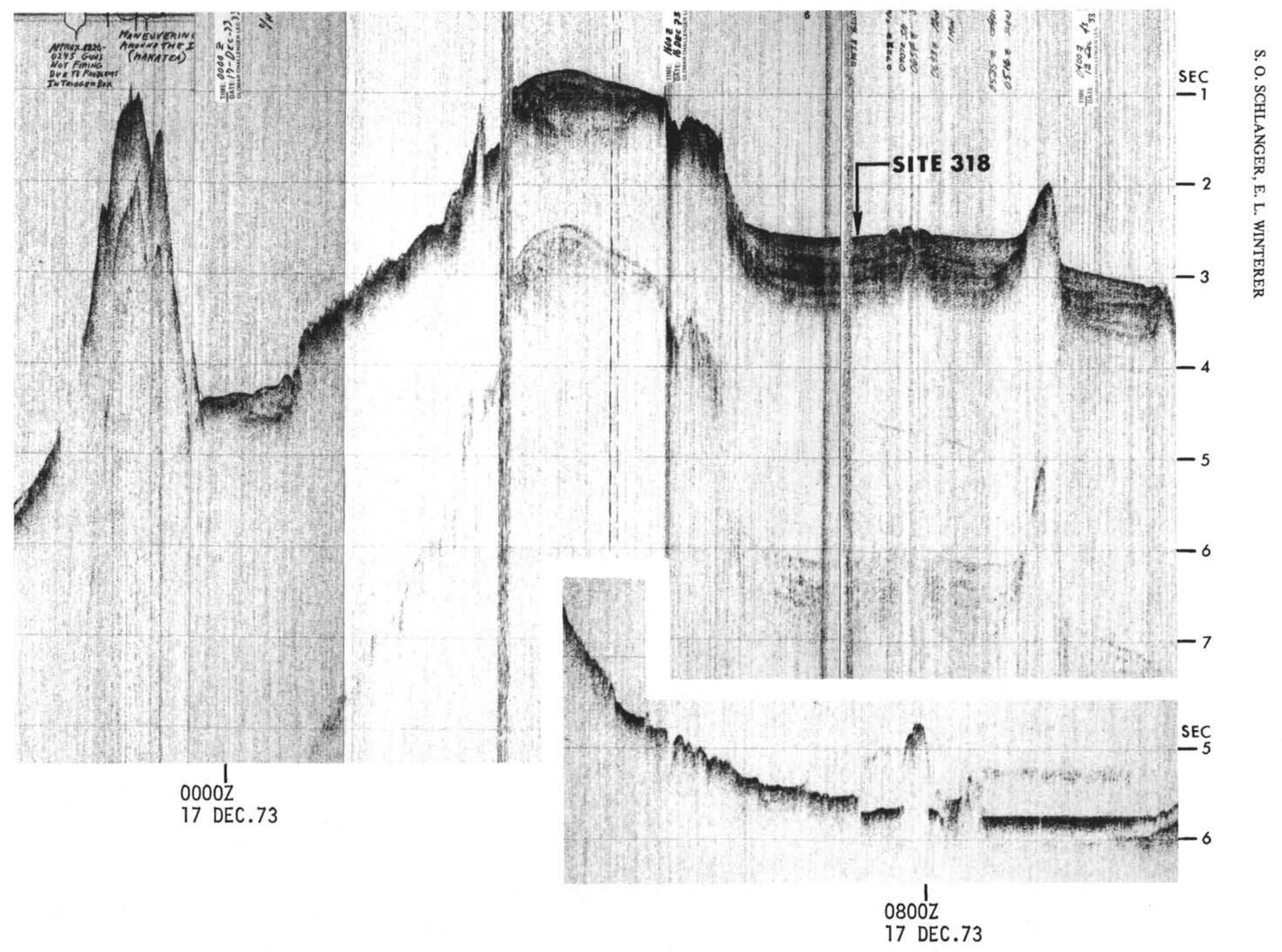

Figure 2. (Continued). 

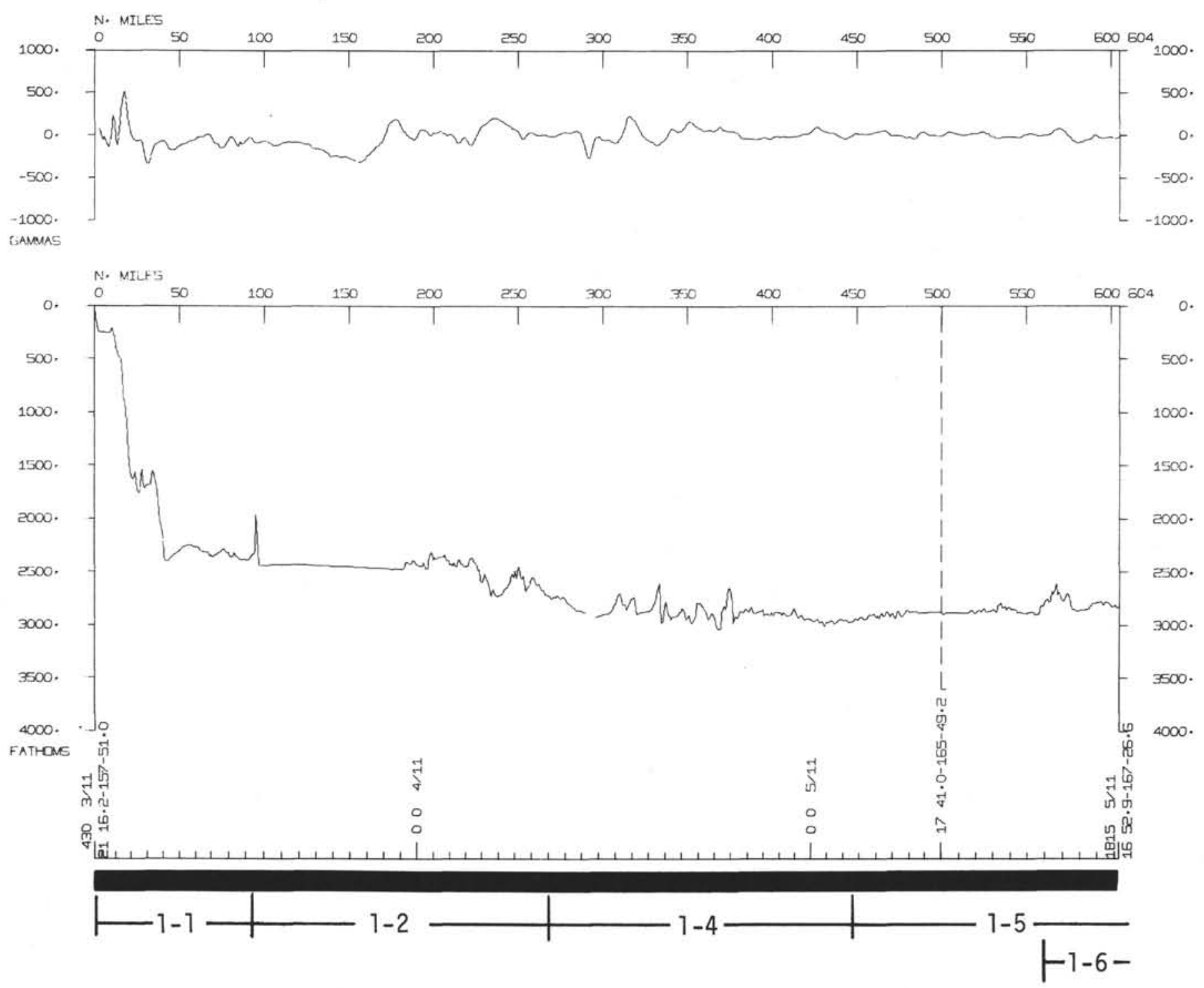

Figure 3. Magnetic and bathymetric profiles from Leg 33 plotted against nautical miles from Honolulu (at top) and time (at bottom); seismic profiles are keyed to the time scale by roll and number.

drilled on Leg 8 (Tracey, Sutton, et al., 1971). At that site the bottom assembly twisted off at 15 meters subbottom depth in mid-Eocene indurated clay and chert which was overlain by mixed Quaternary, Miocene, and Eocene radiolarian clay. Between this point and the ridge that forms the eastern boundary of the Line Islands trough (see Site 314 report) the bottom is characterized by a relatively flat, young, acoustically transparent layer.

The magnetic profile over the Hawaiian Arch has considerable relief, and beyond the arch the topography and magnetic profiles are both smooth. The age of the segment of the Pacific plate between Honolulu and Site 314 is between 90 and 110 m.y. (Larson and Chase, 1972), a time of magnetic stability (Baldwin et al., 1974). At Site 164, located between the Molokai and Clarion fracture zones (Figure 1), drilled on Leg 17 (Winterer, Ewing, et al., 1973), the basement age was found to be 100-115 m.y.

\section{Site 314 to Site 315 (Figure $4 b$ )}

On leaving Site 314 Glomar Challenger steamed southeast parallel to the trend of the Line Islands chain. Sediment cover over acoustic basement, between Site 314 and the intersection of the ship's track with the extension of the Clarion Fracture Zone, is very thin. The effects of deep submarine erosion by bottom waters moving through the Line Islands chain are especially marked between the Clarion Fracture Zone and Site 315. The erosion is ascribed to late Cenozoic Antarctic bottom water activity; erosion at the present time may be negligible (Winterer, in preparation). Normark and Spiess (in press) note that episodic eastward flow of bottom water may be occurring at present with velocities occasionally sufficient to form ripples within the sandy sediments of the moat floor. At 17309 November 1973 $\sim 0.2 \mathrm{sec}$ of sections has been removed. At 070010 November 1973 a deep passage is seen where the entire 


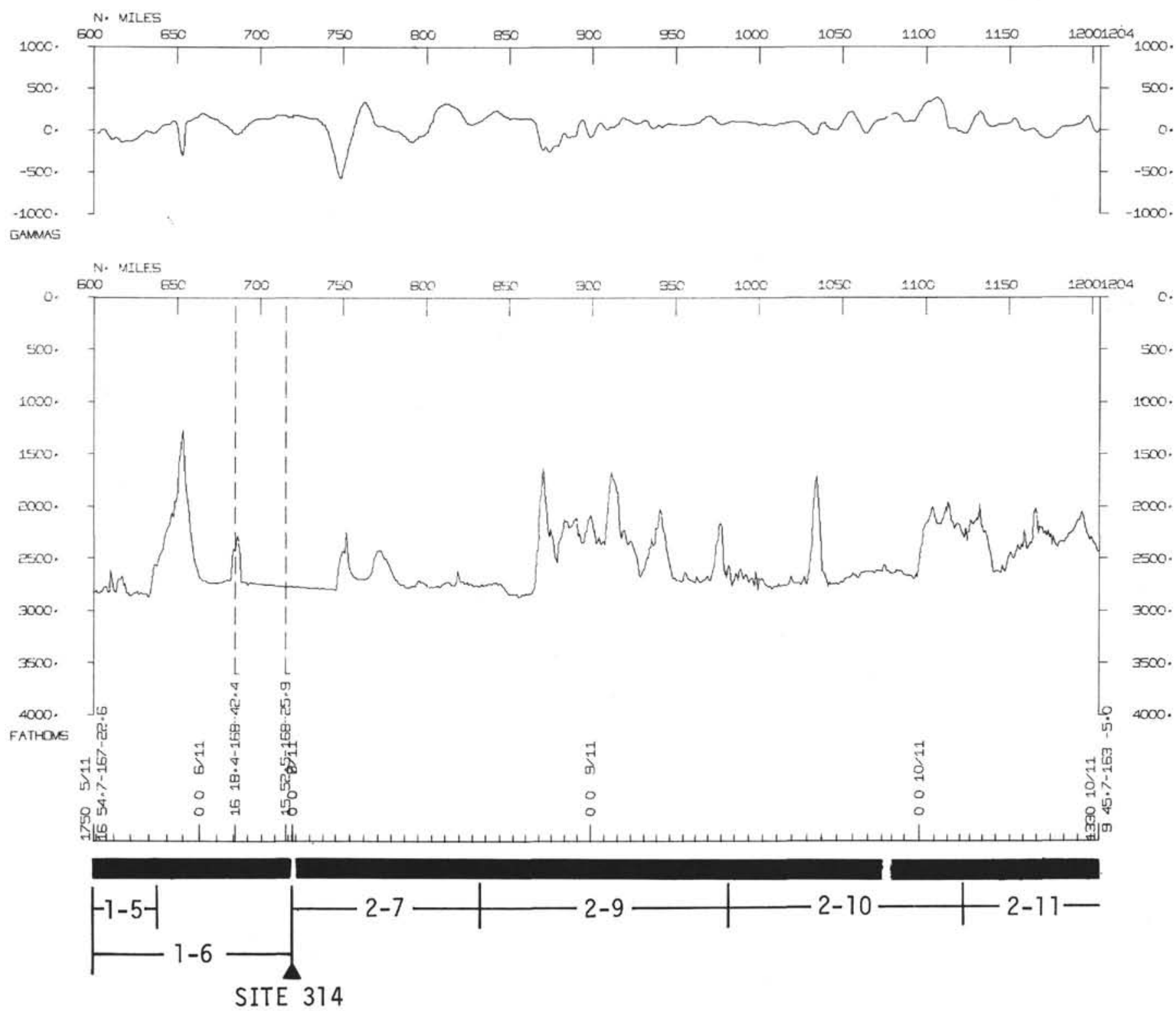

Figure 3. (Continued)

sediment cover over acoustic basement has been removed. Previous surveys across the Line Islands have also revealed such erosional features (Raff, 1973; seismic profile 3 ).

At the latitude of the Kingman Reef-Palmyra Island segment of the Line Islands Ridge (see Figure 1) the thickness of sediments above the 0.70 -sec reflector increases markedly. This increase is probably related to an increase in biological productivity at this latitude. Another factor contributing to the thickness increase may be the numerous topographic highs that reach to within less than 1000 fathoms. Sediments swept from these would form turbidite contributions to the strata. At Site 165, drilled on the west flank of the Line Islands Ridge (Winterer, Ewing, et al., 1973) turbidites make up a significant fraction of the sediment column. The morphology of the sediments in the troughs from 0000 12 November 1973 to 160012 November 1973 indicates concentric draping of the sediments suggestive of a dominantly pelagic mode of deposition. The internal reflectors also drape over "basement" highs and maintain a rough parallelism. This "drape" morphology contrasts with the flat-lying character of dominantly turbidite sections.

From 000012 November 1973 to Site 315 the reflector stratigraphy is quite coherent. The 0.70 -sec reflector that correlates with the Paleocene-Oligocene cherty chalk - Miocene chalk contact is a dominant feature; below this, the sequence is vague and the basalt "basement" may not be clearly recorded. Perhaps the high velocity limestones near the base of the sedimentary section form an acoustic mask over the basalt. Above the 0.70 -sec reflector somewhat discontinuous reflectors such as the $0.40 \mathrm{sec}$ may be related to ooze-chalk boundaries.

\section{Site 315 to Site 316 (Figure 4c)}

On leaving Site 315 we steamed to Fanning Island to drop off personnel and pick up spare parts. Buttressing 

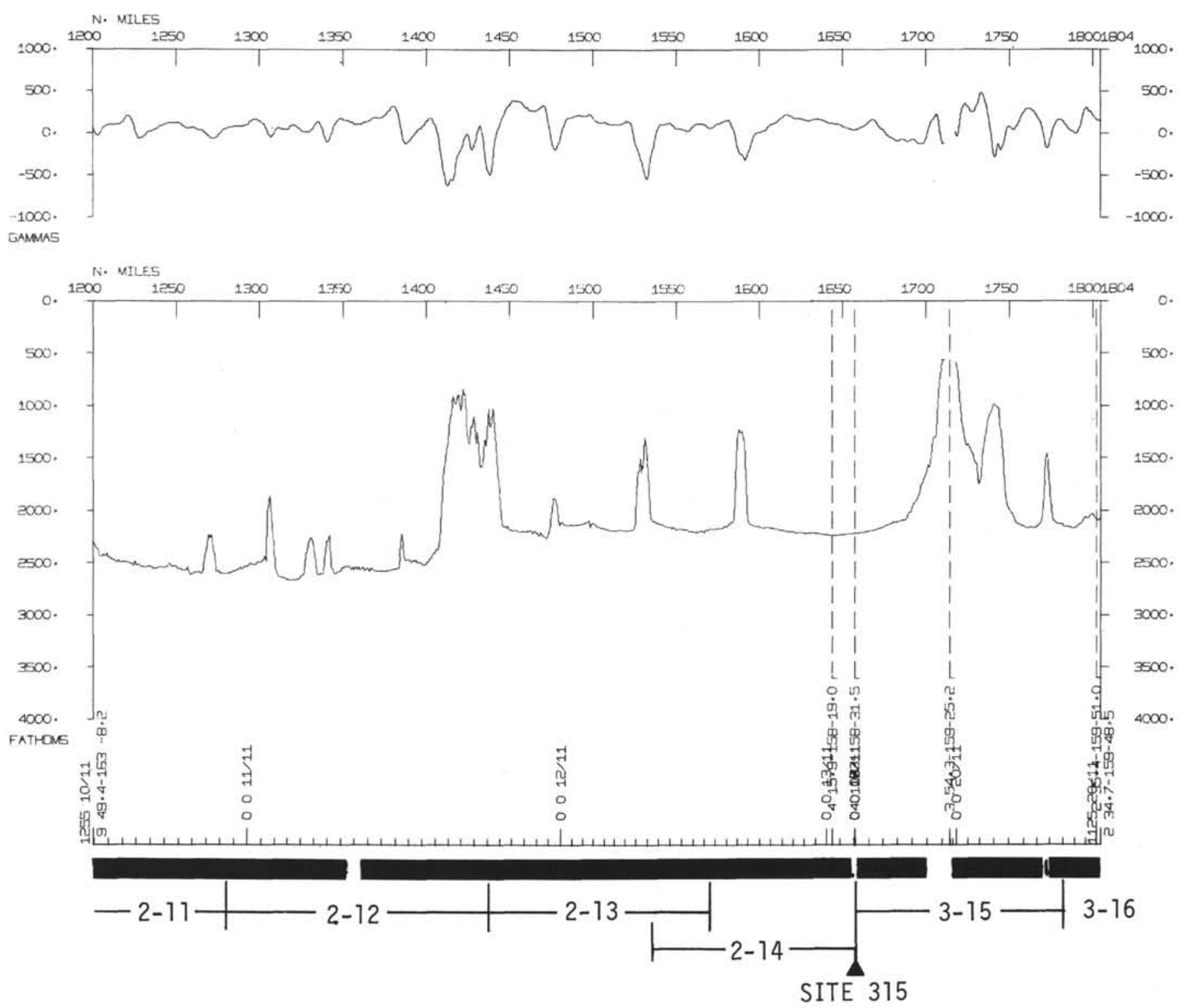

Figure 3. (Continued)

of deep reflectors against the Line Islands Ridge is apparent (1500 to 200019 November 1973). On leaving Fanning Island we crossed over a deep submarine canyon that drains to the west $(020020$ November 1973) from shelf depths around the southwest side of the island. In this area such drainages cut as deeply as $2 \mathrm{~km}$ into the archipelagic apron (Winterer, this volume). Between 1730 and 232320 November 1973 the ship's track crossed over the extension of the Nova-Canton trough. There, the section has been cut down to possibly the 0.475 -sec reflector at Site 316 , which is placed at a hiatus between Oligocene cherty chalk, dolomite and limestone, and Miocene chalk; the 0.70 -sec reflector at Site 315 may correlate with the Site 3160.475 reflector. A striking feature of the Christmas Island Trough is the systematic thinning of the post 0.475 -sec reflector section. Individual reflectors can be traced virtually without interruption for $24 \mathrm{hr}$ of steaming time. Basalt base- ment was not reached at this site, and there does not appear to be a definite reflector below the 0.71-sec reflector within the Cretaceous limestone section.

\section{Site 316 to Site 317 (Figure 4d)}

On leaving Site 316 we steamed south-southwest in order to make the shortest distance to intersect the Cato3 seismic profile along which our planned Site 317 lay. The well-stratified section of Tertiary carbonates so prominent around the Line Islands thinned rapidly as the water deepened; internal reflectors also disappeared. By 053028 November even the very thin, "transparent" layer had virtually disappeared as we crossed the northern part of the Central Basin. The thinning and disappearance of the internal reflectors is probably due to the systematic lessening with increasing depth of $\mathrm{CaCO}_{3}$ content of the Tertiary section. 

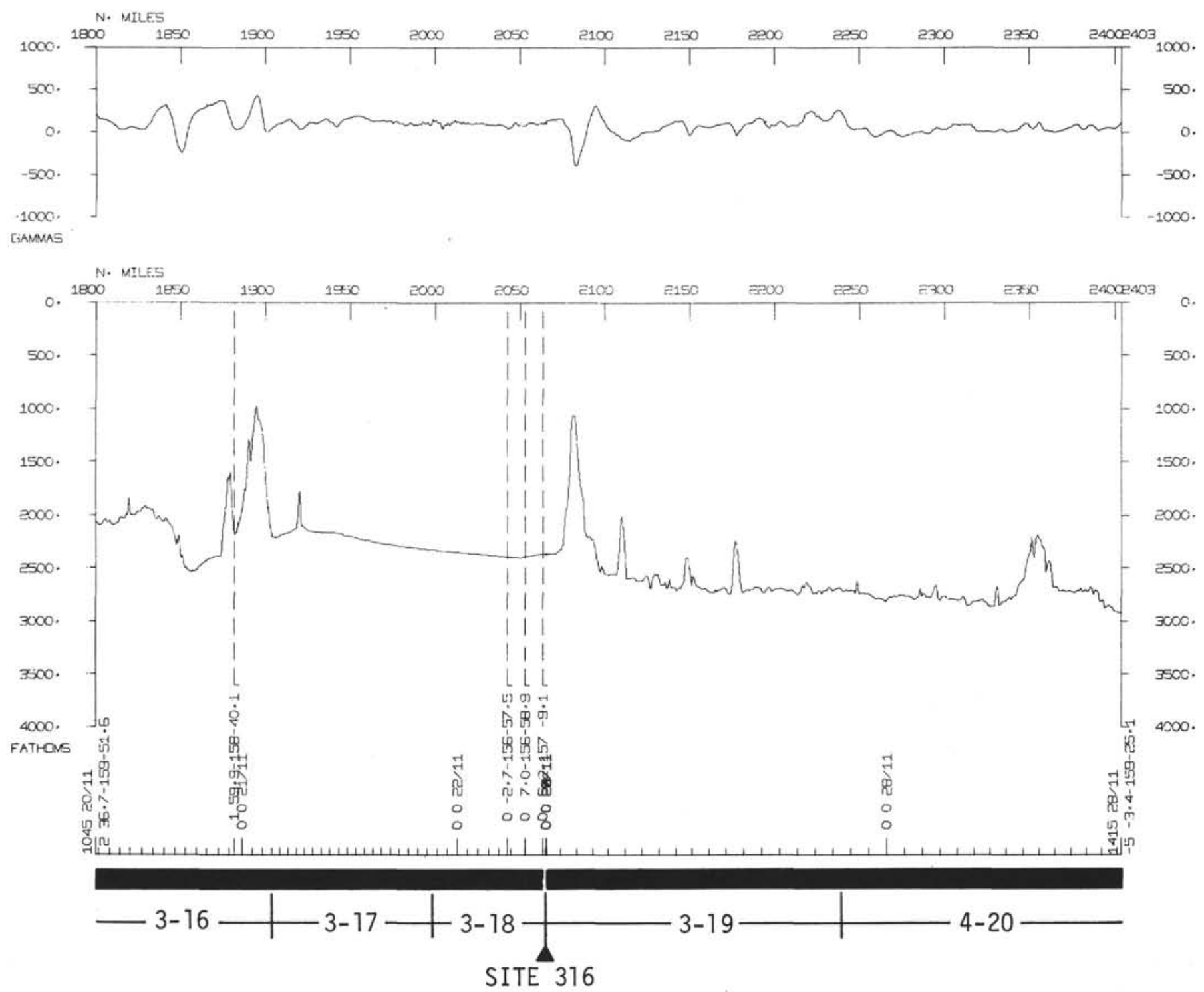

Figure 3. (Continued)

From 133028 November to $\sim 220029$ November the magnetic anomaly pattern is quite regular (Figure 3e); the pattern is very flat over the Manihiki Plateau.

At 103029 November the northwest boundary faults of the Manihiki Plateau were crossed. The water depth decreases in stepwise fashion as the plateau is approached and the sedimentary section thickens markedly. The section is locally influenced by deep erosional scars and probably local slumping. The topography and structural geology of the plateau is discussed in detail by Winterer et al. (1974). Their fig. 5 shows the Cato-3 profile used by us in planning the approach to Site 317; a detailed discussion will not be repeated here since our profile duplicates the Cato-3 line.

\section{Site 317 to Site 318 (Figure 4e)}

Leaving Site 317 Glomar Challenger steamed eastsoutheast over the eastern boundary faults of the Manihiki Plateau. Our course was approximately $25^{\circ}$ more easterly than the Cato-3 track discussed by
Winterer et al. (1974) but similar features were seen. A buried ridge over which the sediments have draped and compacted and upon which moating has taken place is prominent. Very deep erosion, probably down to the mid-Eocene cherty chalk (noted by "C" on Figure 4c) can be clearly seen. The basement rim is well marked as are the numerous faults.

The thickness of the pre-Eocene strata appears to be constant although the water depth increases markedly from 04558 December to 12008 December suggesting that the relief along this side of the plateau developed in post-Cretaceous time.

The Penrhyn Basin has a vanishingly thin sedimentary cover similar to the situation in the Central Basin between Sites 316 and 317. The sediments thicken systematically as the Tuamotu Ridge system is approached.

Approaching the Tuamotu Ridge our course was eastsoutheast (approximately $100^{\circ}$ ). Just northeast of our proposed Site 318 the Marquesas Fracture Zone trends 

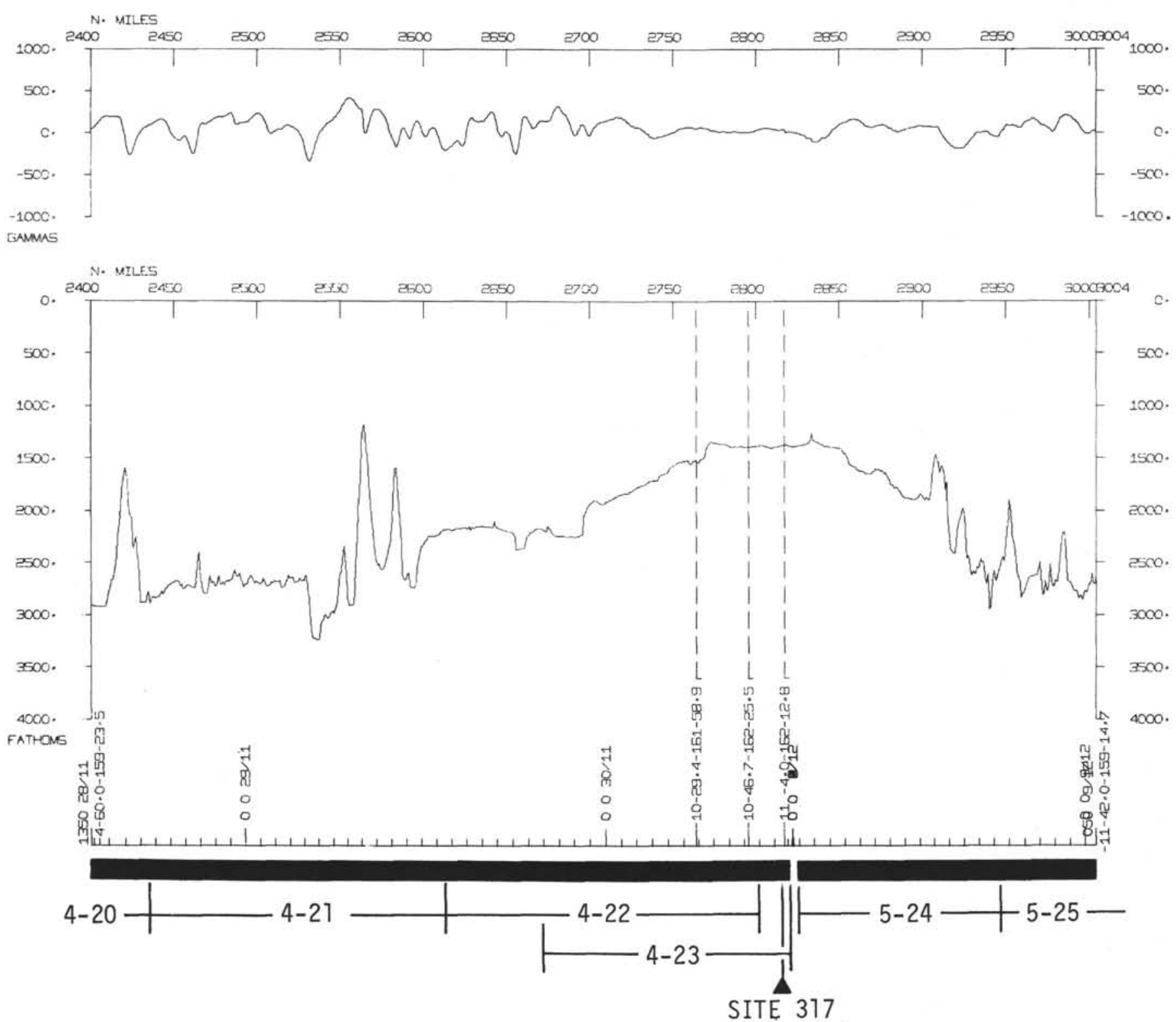

Figure 3. (Continued)

slightly south or west. We were therefore steaming at a very low angle to the fracture zone. The ridge crossed between 0900 and 183012 December probably is an unmapped extension of the Marquesas Fracture Zone. At 020013 December we crossed the scarp that borders the Tuamotu Ridge on its north side and crossed the perched, sediment-filled basin in which Site 318 is located. The internal reflector stratigraphy in this perched basin is irregular and vague, possibly due to the high percentage of the section that is turbiditic in nature. No attempt was made at this site to correlate the seismic profile with the drilling results.

\section{Site 318 to Papeete (Figure 4f)}

On leaving Site 318 Glomar Challenger steamed through the pass between the large atolls of Rangiroa and Arutua marked by $0.3+\mathrm{sec}$ of well-stratified sediment. Because we had a few hours of available steaming time we passed by Makatea Island thus crossing a possi- ble fault zone between the subsiding line of the Tuamotu atolls and the emergent limestone island of Makatea, which has the morphology of an uplifted atoll (Chevalier, 1973).

The seismic gear was pulled aboard as we approached Tahiti and Leg 33 ended.

\section{REFERENCES}

Baldwin, B., Coney, P.J., and Dickenson, W.R., 1974. Dilemma of a Cretaceous time scale and rates of sea-floor spreading: Geology, v. 2, p. 267-272.

Chevalier, J.P., 1973. Geomorphology and geology of Coral Reefs in French Polynesia. In Jones, O.A. and Endean, R. (Eds.), Biology and geology, Coral Reefs: v. 1, New York (Academic Press).

Larson, R.L. and Chase, C.G., 1972. Late Mesozoic evolution of the western Pacific Ocean: Geol. Soc. Am. Bull., v. 83, p. 3627-3644. 

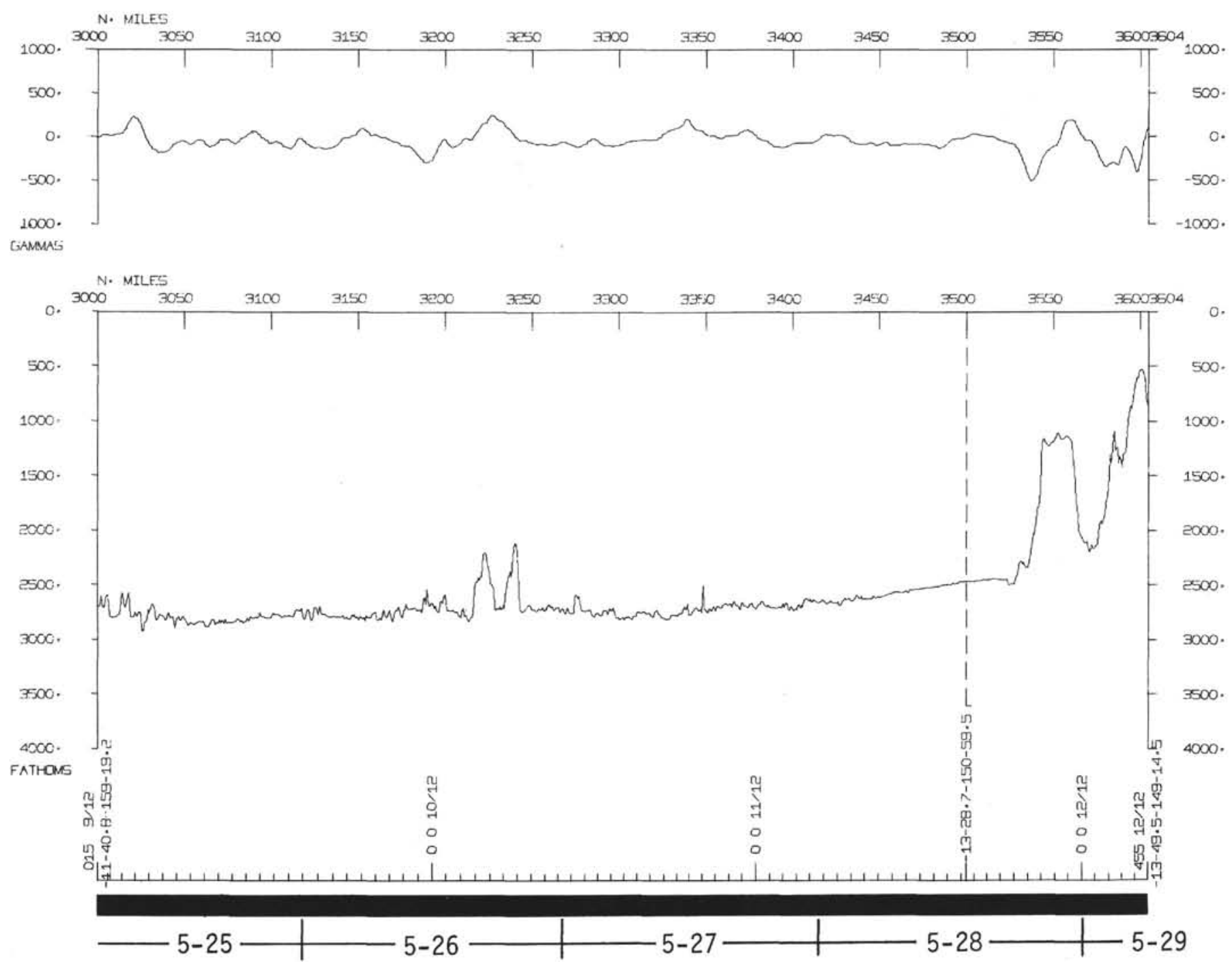

Figure 3. (Continued)

Normark, W.R. and Spiess, F.N., in press. Erosion in the Line Islands archipelago apron: effect of small-scale topographic relief: Geol. Soc. Am. Bull.

Raff, A.D., 1973. Underway Data. In Winterer, E.L., Ewing, J., et al., Initial Reports of the Deep Sea Drilling Project, Volume 17: Washington (U.S. Government Printing Office), p. 337-364.

Stearns, H.T., 1939. Geologic map and guide of Oahu, Hawaii: Terr. of Hawaii, Hydrographic Division, Bull., No. 2 .
Tracey, J.I., Jr., Sutton, G.H., et al., 1971. Initial Reports of the Deep Sea Drilling Project, Volume 7: Washington (U.S. Government Printing Office).

Winterer, E.L., Lonsdale, P.F., Matthews, J.L., and Rosendahl, B.R., 1974. Structure and acoustic stratigraphy of the Manihiki Plateau: Deep-Sea Res., v. 21, p. 798-814.

Winterer, E.L., Ewing, J., et al., 1973. Initial Reports of the Deep Sea Drilling Project, Volume 17: Washington (U.S. Government Printing Office). 

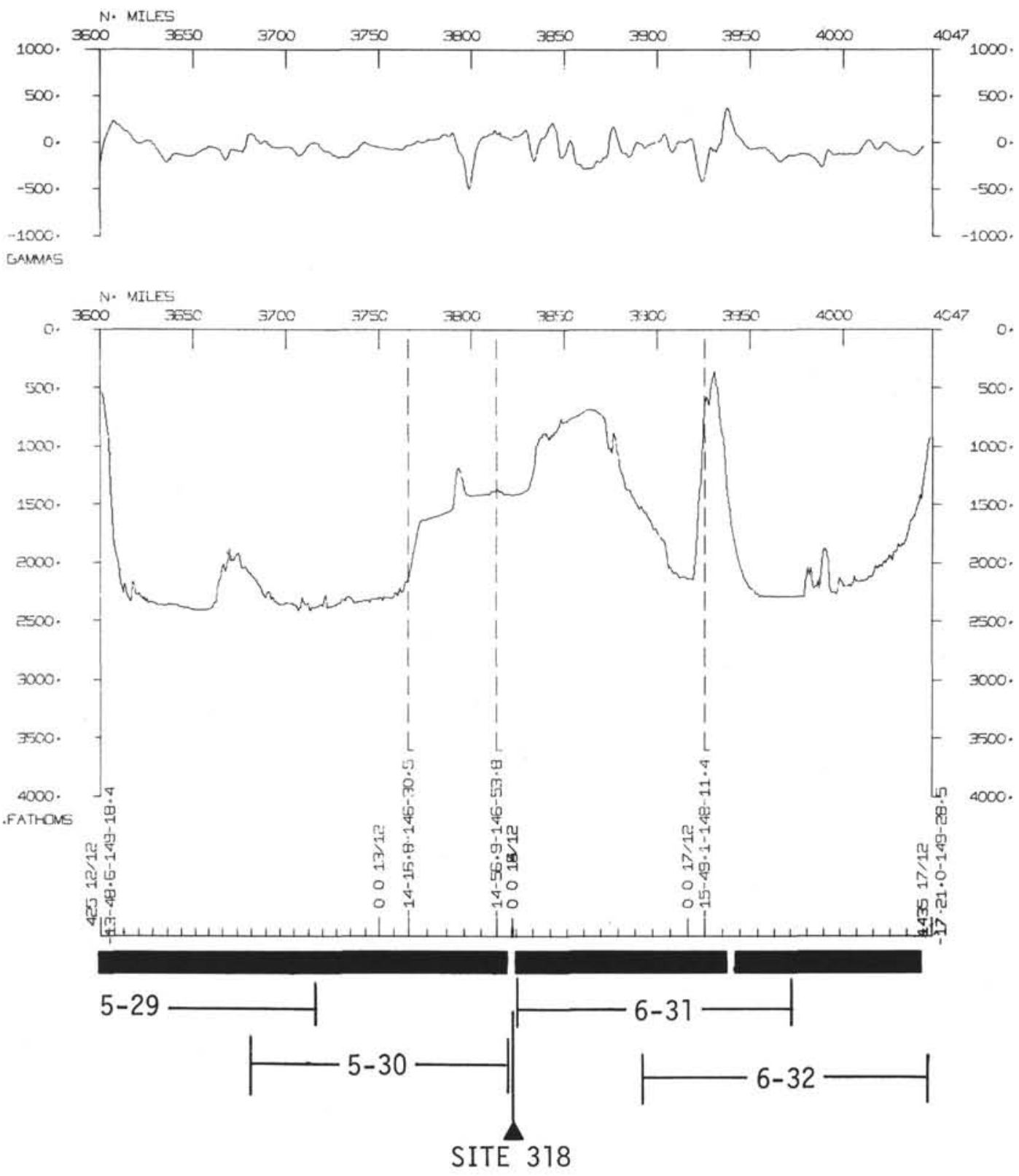

Figure 3. (Continued). 


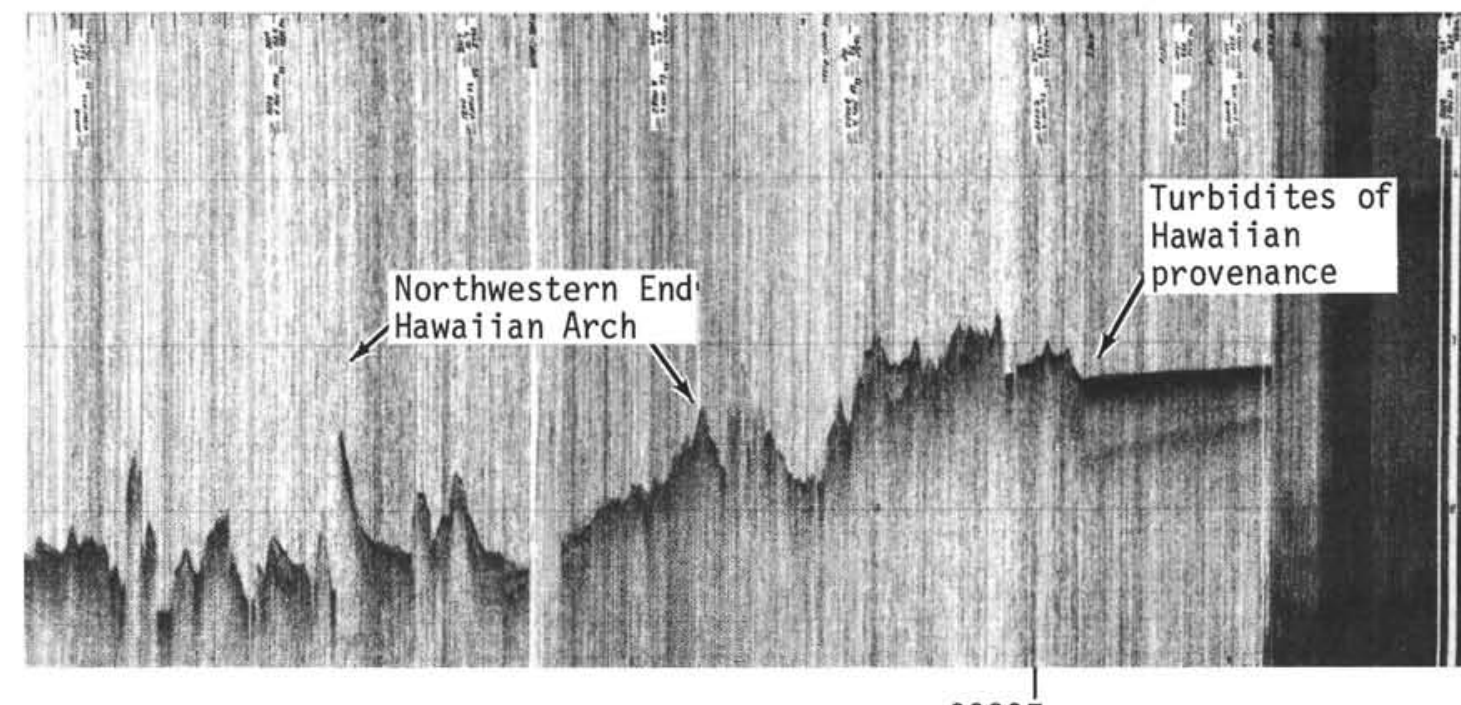

$0000 \mathrm{Z}$

4 Nov. ,73

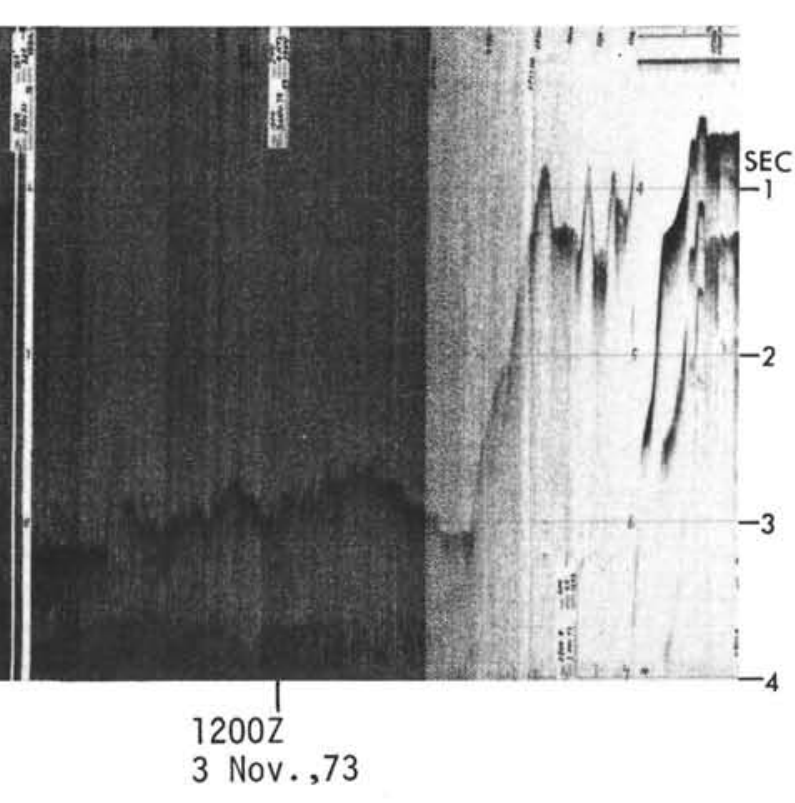

SEC

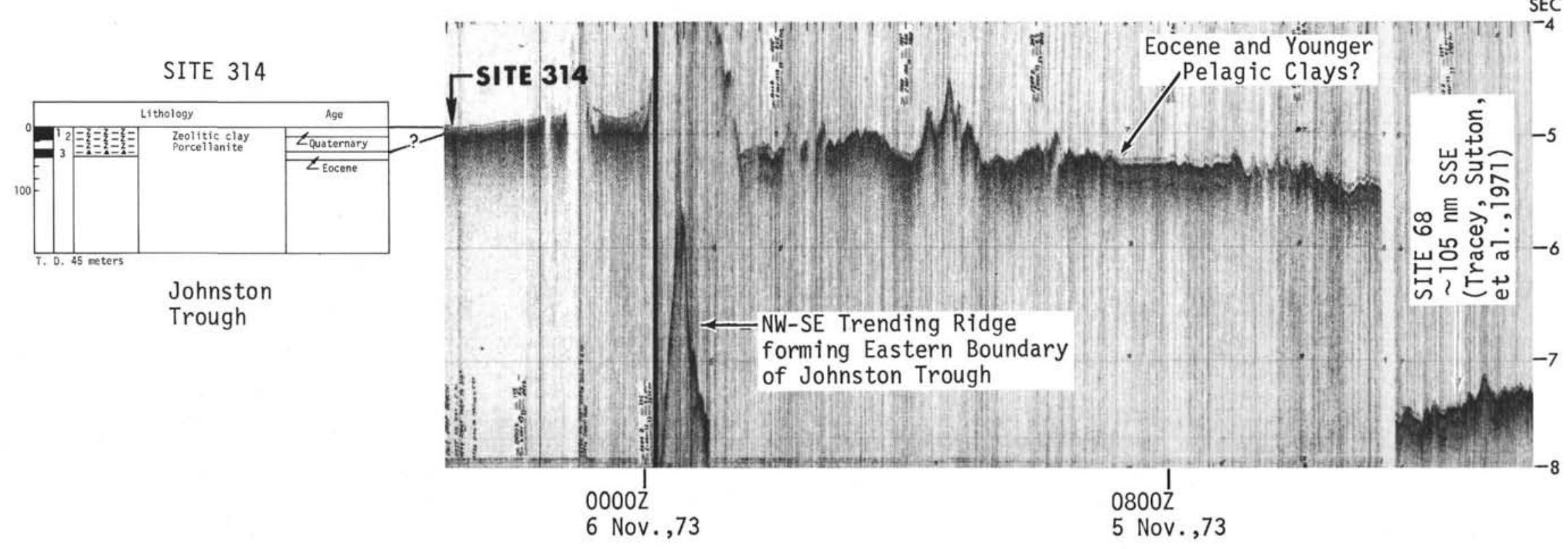

Figure 4. Seismic profiles taken on Leg 33 on the No. 2 EDO kept set for a 4-sec sweep. Annotated in terms of drill-site stratigraphy, acoustistratigraphy, physiography, and structure. 

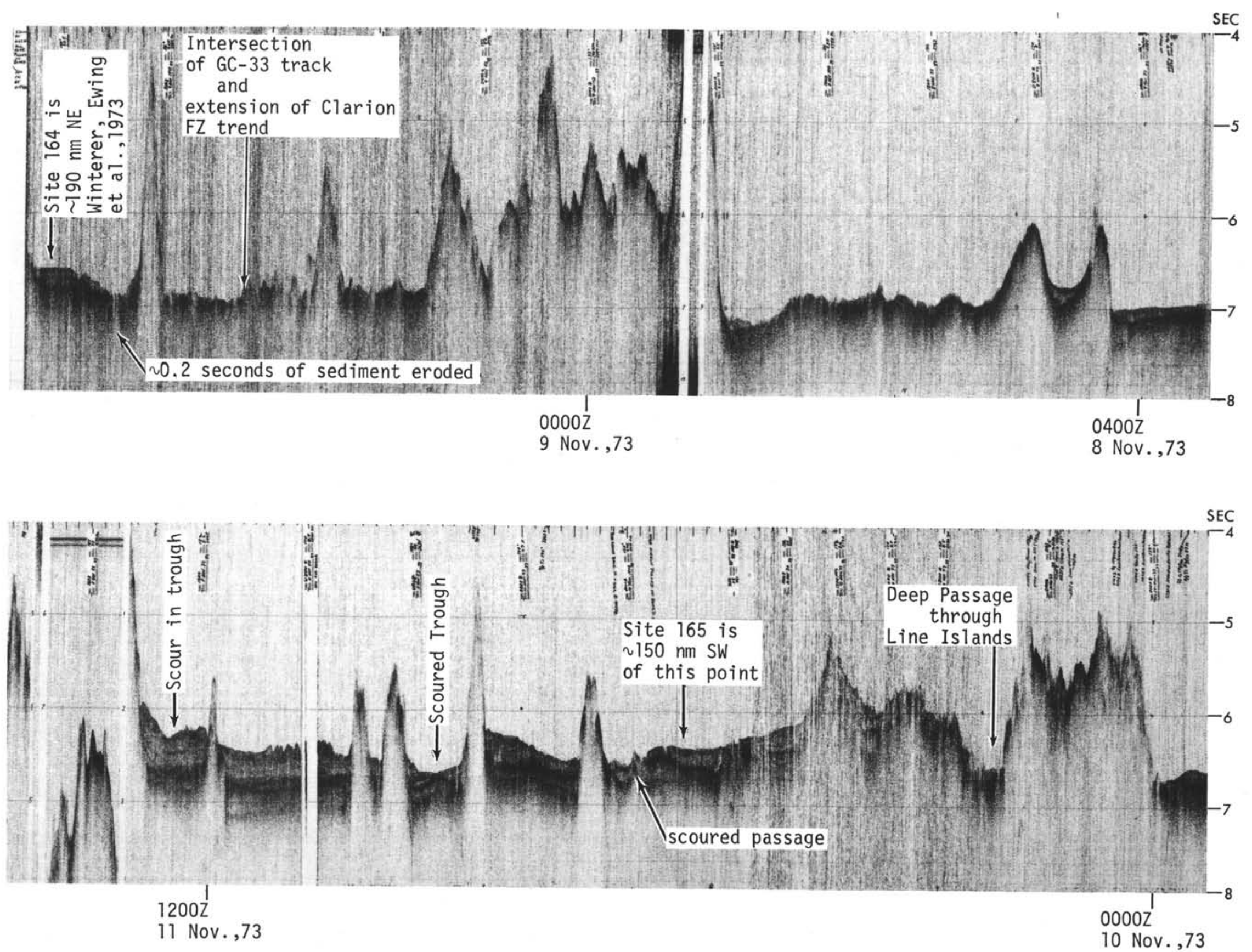

Figure 4. (Continued) 


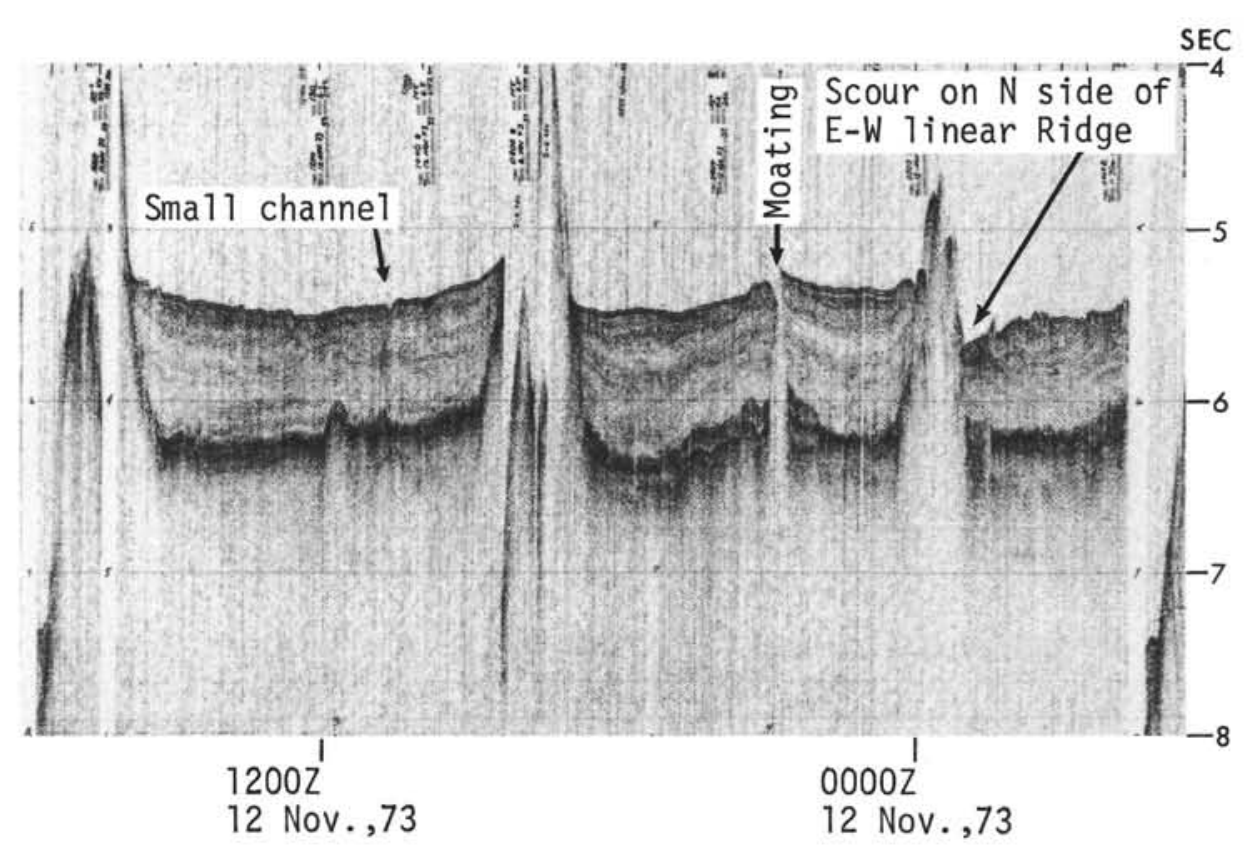

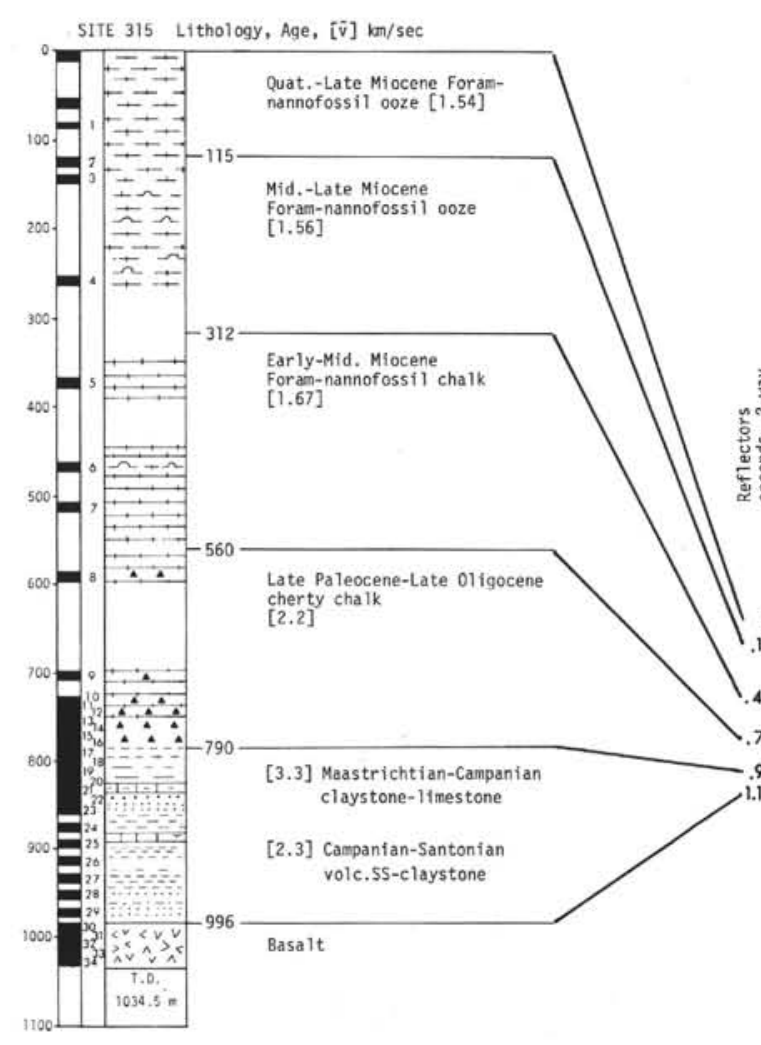

Figure 4. (Continued)

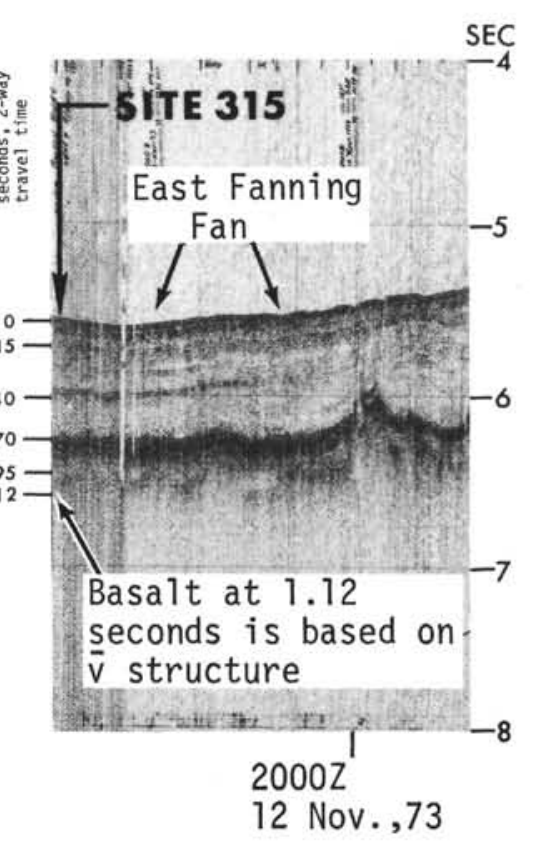

12 Nov. ,73 

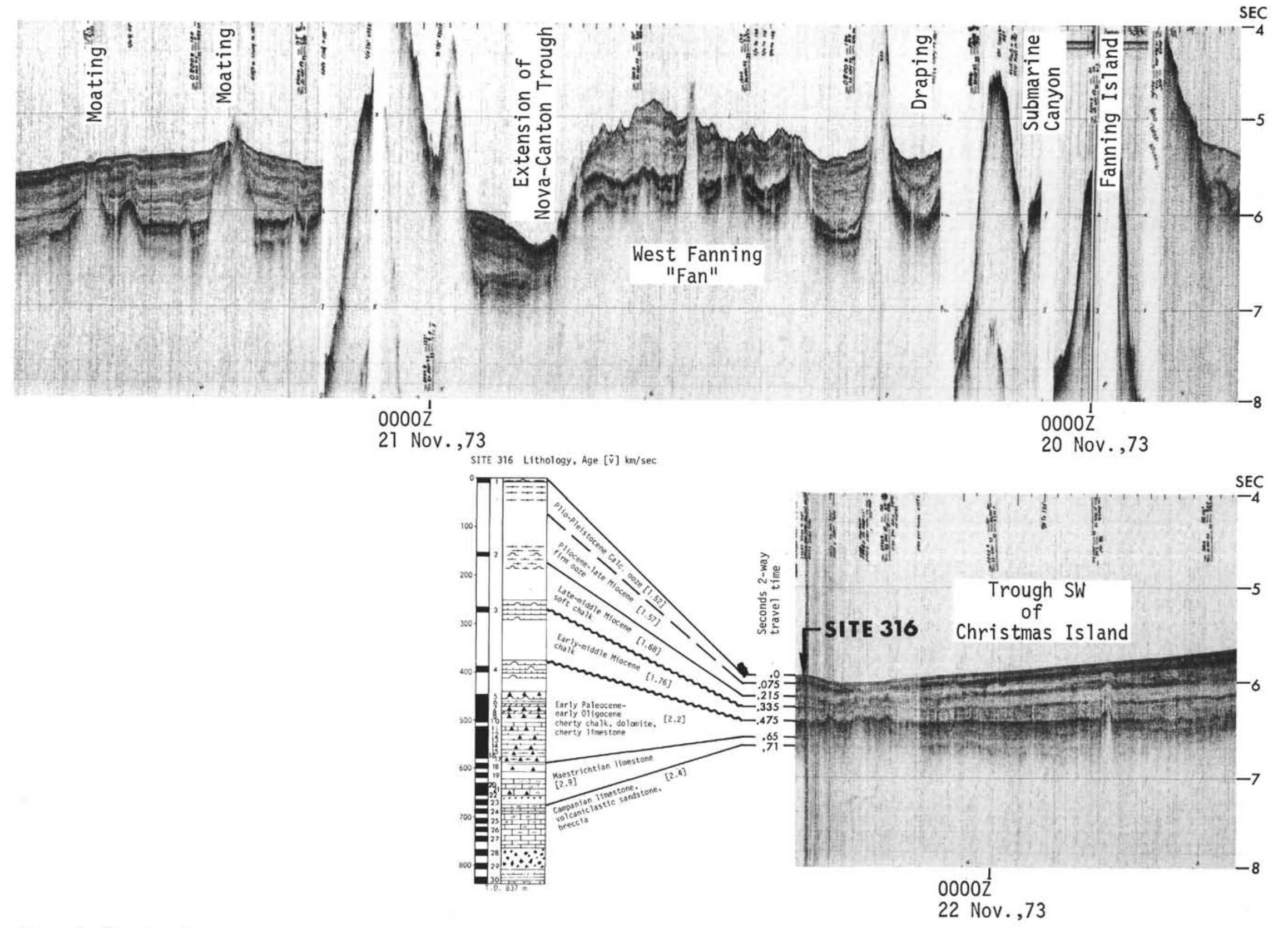

Figure 4. (Continued) 

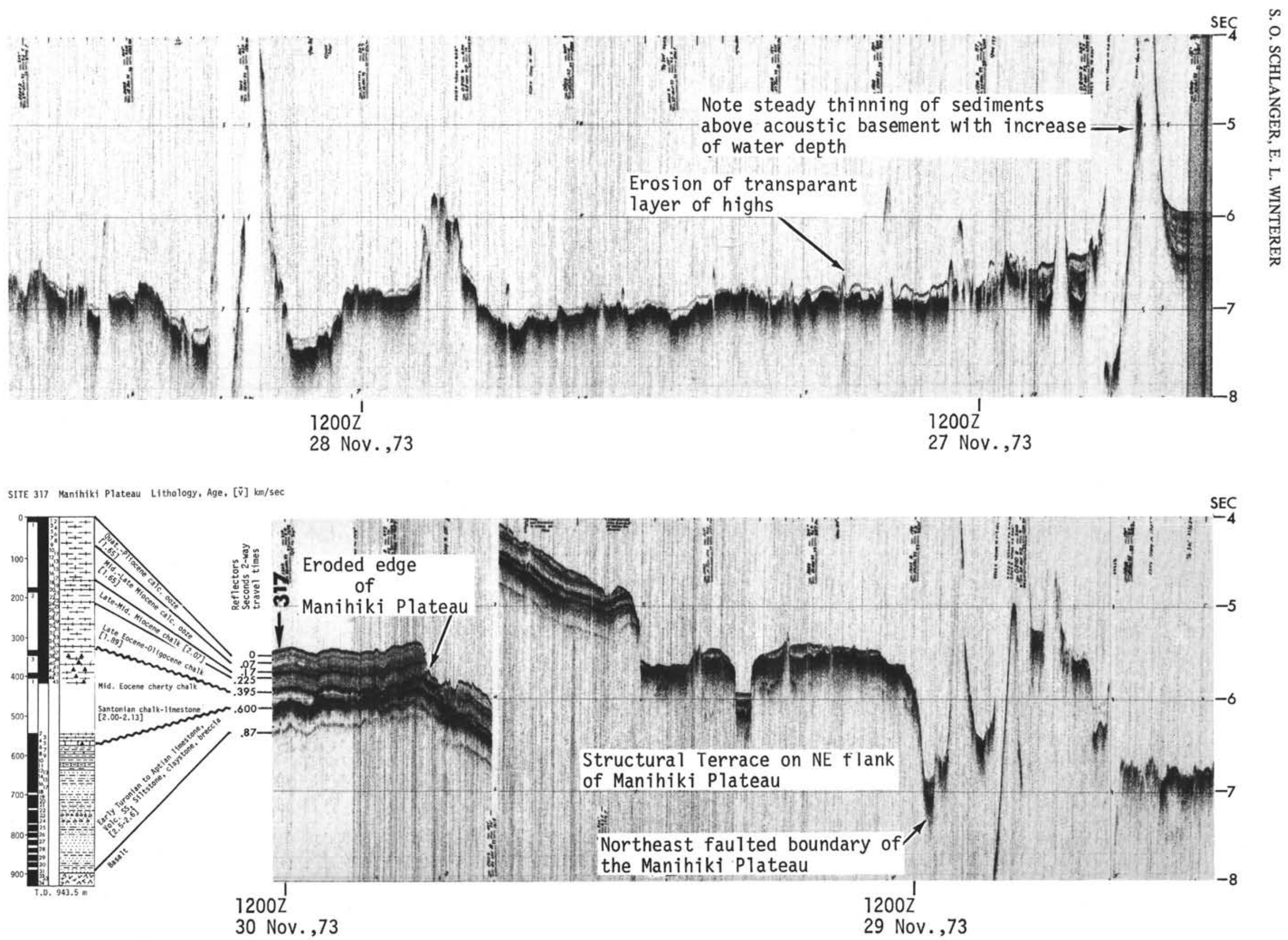

Figure 4. (Continued) 

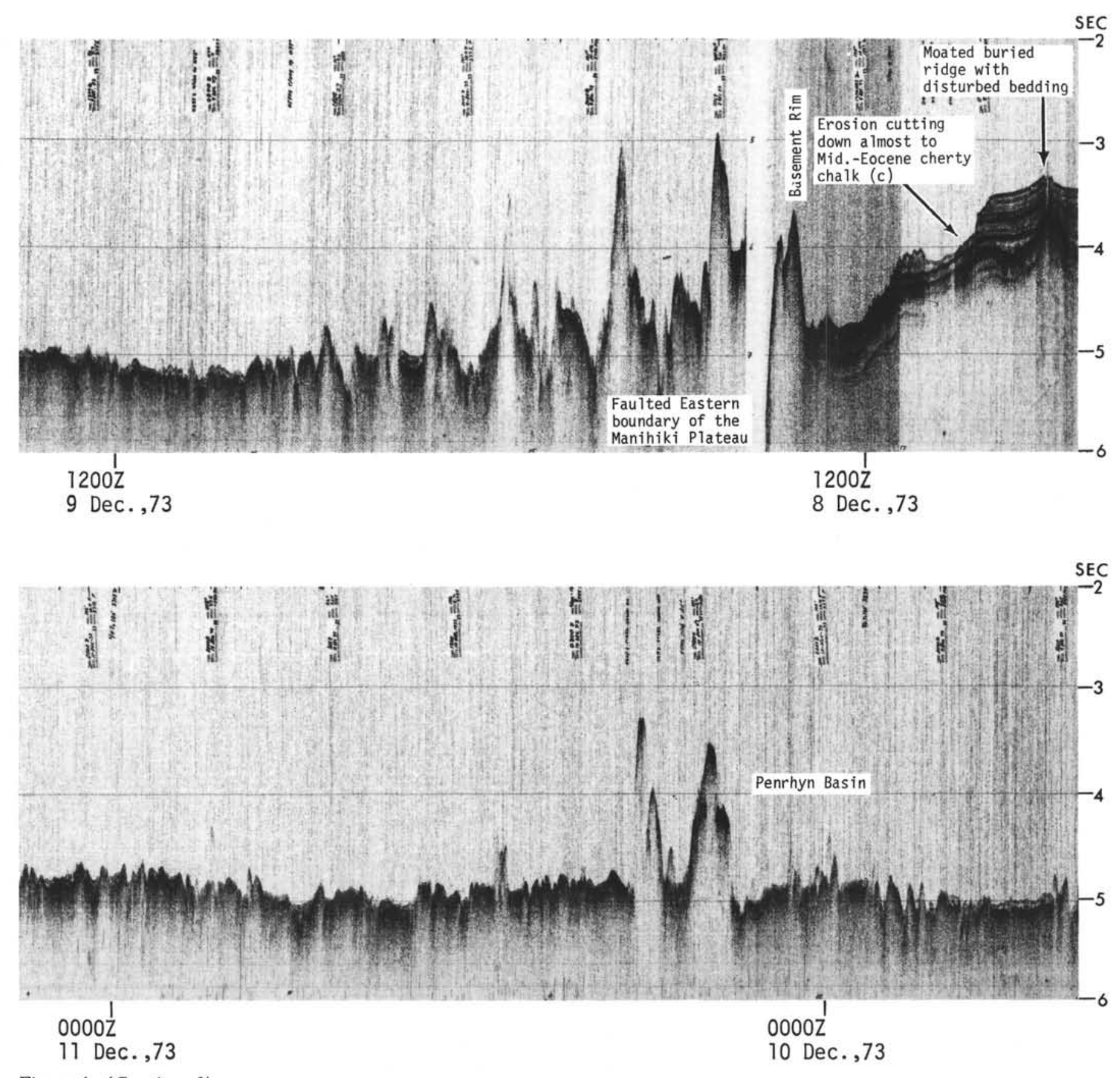

Figure 4. (Continued) 

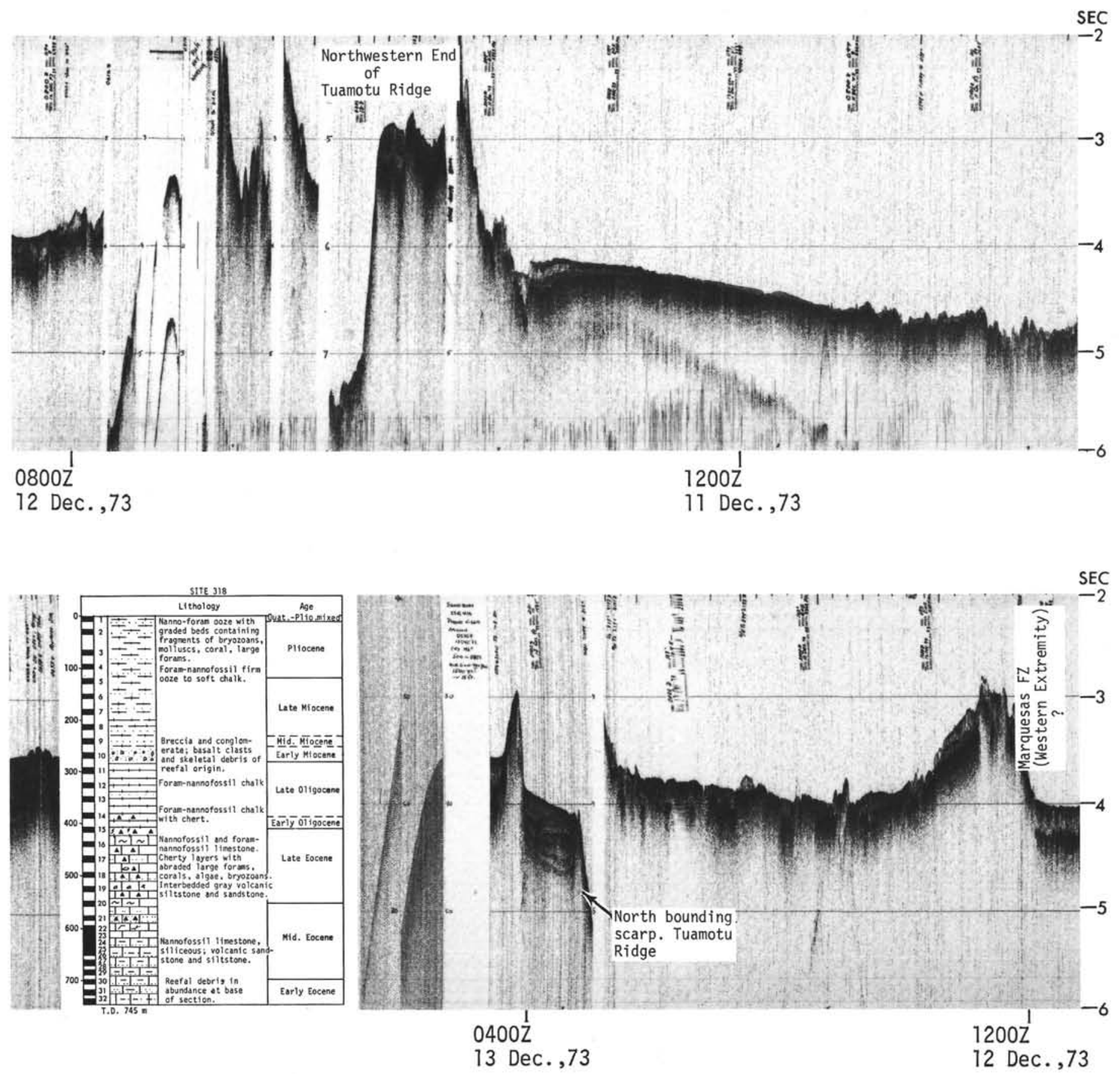

Figure 4. (Continued) 


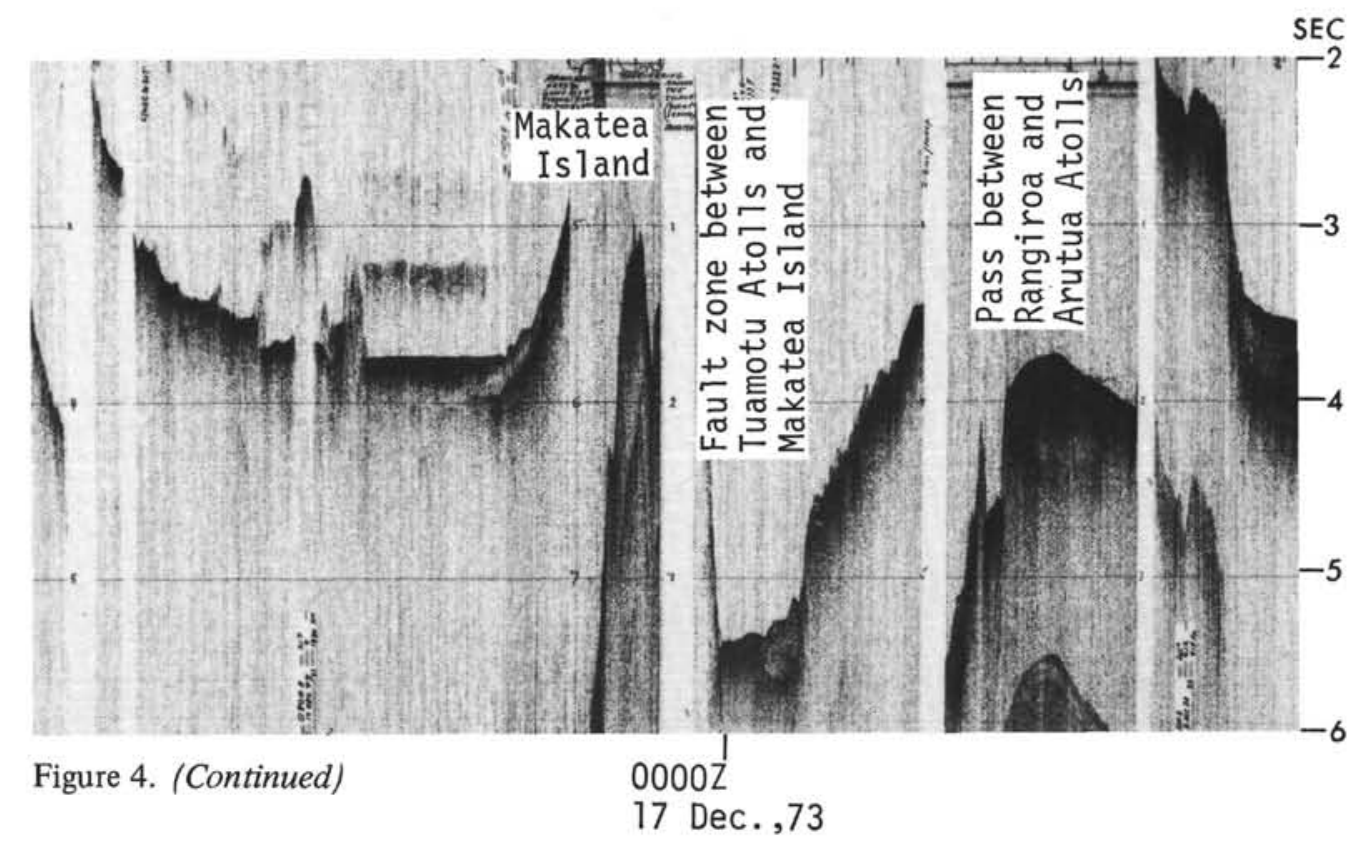

